\title{
Broad-band high-resolution rotational spectroscopy for laboratory astrophysics ${ }^{\star}$
}

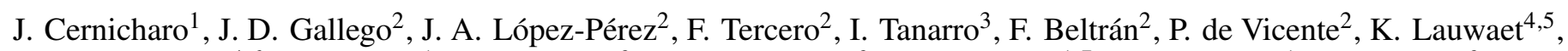
B. Alemán ${ }^{4,6}$, E. Moreno ${ }^{1}$, V. J. Herrero ${ }^{3}$, J. L. Doménech ${ }^{3}$, S. I. Ramírez ${ }^{1,7}$, C. Bermúdez ${ }^{1}$, R. J. Peláez ${ }^{3}$, M. Patino-Esteban ${ }^{2}$, I. López-Fernández ${ }^{2}$, S. García-Álvaro ${ }^{2}$, P. García-Carreño ${ }^{2}$, C. Cabezas ${ }^{1}$, I. Malo ${ }^{2}$, R. Amils ${ }^{2}$, J. Sobrado ${ }^{8}$, C. Diez-González ${ }^{2}$, J. M. Hernandéz ${ }^{2}$, B. Tercero ${ }^{2}$, G. Santoro ${ }^{4}$, L. Martínez ${ }^{4}$, M. Castellanos ${ }^{1}$, B. Vaquero Jiménez ${ }^{2}$, J. R. Pardo ${ }^{1}$, L. Barbas ${ }^{2}$, J. A. López-Fernández ${ }^{2}$, B. Aja ${ }^{9}$, A. Leuther ${ }^{10}$, and J. A. Martín-Gago ${ }^{4}$

\footnotetext{
${ }^{1}$ Instituto de Física Fundamental (IFF. CSIC). Group of Molecular Astrophysics, C/ Serrano 123, 28006 Madrid, Spain e-mail: jose.cernicharo@csic.es

2 Centro de Desarrollos Tecnológicos, Observatorio de Yebes (IGN), 19141 Yebes, Guadalajara, Spain

3 Instituto de Estructura de la Materia (IEM. CSIC), Molecular Physics Department, C/Serrano 123, 28006 Madrid, Spain

${ }^{4}$ Instituto de Ciencia de Materiales de Madrid (ICMM. CSIC). Materials Science Factory. Structure of Nanoscopic Systems Group, ESISNA., C/ Sor Juana Inés de la Cruz 3, 28049 Cantoblanco, Madrid, Spain

5 IMDEA Nanociencia, Ciudad Universitaria de Cantoblanco, 28049 Cantoblanco, Madrid, Spain

${ }^{6}$ IMDEA Materiales, C/Eric Kandel 2, Tecnogetafe, 28906 Getafe, Madrid, Spain

7 Centro de Investigaciones Químicas, UAEM. Av. Universidad, 1001 Col. Chamilpa, 62209 Cuernavaca, Morelos, Mexico

8 Centro de Astrobiología (CSIC-INTA), Crta. de Torrejón de Ardoz a Ajalvir km 4., Torrejón de Ardoz 28850, Spain

9 Universidad de Cantabria, Department of Communication Engineering, Avda. Los Castros, 39005 Santander, Spain

${ }^{10}$ Fraunhofer Institut fur Angewandte Festkorperphysik, Tullastresse 72, 79108 Freiburg, Germany
}

Received 4 February 2019 / Accepted 14 March 2019

\begin{abstract}
We present a new experimental set-up devoted to the study of gas phase molecules and processes using broad-band high spectral resolution rotational spectroscopy. A reactor chamber is equipped with radio receivers similar to those used by radio astronomers to search for molecular emission in space. The whole range of the $Q(31.5-50 \mathrm{GHz})$ and $W$ bands $(72-116.5 \mathrm{GHz})$ is available for rotational spectroscopy observations. The receivers are equipped with $16 \times 2.5 \mathrm{GHz}$ fast Fourier transform spectrometers with a spectral resolution of $38.14 \mathrm{kHz}$ allowing the simultaneous observation of the complete $Q$ band and one-third of the $W$ band. The whole $W$ band can be observed in three settings in which the $Q$ band is always observed. Species such as $\mathrm{CH}_{3} \mathrm{CN}, \mathrm{OCS}_{\text {, and } \mathrm{SO}}$ are detected, together with many of their isotopologues and vibrationally excited states, in very short observing times. The system permits automatic overnight observations, and integration times as long as $2.4 \times 10^{5} \mathrm{~s}$ have been reached. The chamber is equipped with a radiofrequency source to produce cold plasmas, and with four ultraviolet lamps to study photochemical processes. Plasmas of $\mathrm{CH}_{4}, \mathrm{~N}_{2}, \mathrm{CH}_{3} \mathrm{CN}, \mathrm{NH}_{3}, \mathrm{O}_{2}$, and $\mathrm{H}_{2}$, among other species, have been generated and the molecular products easily identified by the rotational spectrum, and via mass spectrometry and optical spectroscopy. Finally, the rotational spectrum of the lowest energy conformer of $\mathrm{CH}_{3} \mathrm{CH}_{2} \mathrm{NHCHO}$ (N-ethylformamide), a molecule previously characterized in microwave rotational spectroscopy, has been measured up to $116.5 \mathrm{GHz}$, allowing the accurate determination of its rotational and distortion constants and its search in space.
\end{abstract}

Key words. molecular data - molecular processes - line: identification - plasmas - methods: laboratory: molecular

\section{Introduction}

The study of the chemical, physical, and dynamical evolution of molecular clouds, where stars and their planets form, is performed through the observation of the thermal emission or absorption produced by the rotational transitions of the molecules formed in these objects. Large single-dish radiotelescopes or interferometers equipped with very sensitive receivers are used for these purposes. Today's radio astronomical receivers permit broad instantaneous frequency coverage (several $\mathrm{GHz}$ ) that can be fully analysed with fast Fourier transform spectrometers (FFTs) that provide spectral resolutions as low as a few $\mathrm{kHz}$.

\footnotetext{
$\star$ Tables C. 1 and C. 2 are only available at the CDS via anonymous ftp to cdsarc.u-strasbg. fr (130.79.128.5) or via http://cdsarc. u-strasbg.fr/viz-bin/qcat?J/A+A/626/A34
}

Within the ERC project NANOCOSMOS ${ }^{1}$. we proposed using radio astronomical receivers as detectors for molecular spectroscopy and chemical reactivity experiments in a gas cell. Different experiments have been performed in the past based on similar ideas (Hüberts 2006; Ren et al. 2010; Neumaier et al. 2014; Wehres et al. 2018a,b), in particular for the SWAS satellite for which an OCS gas cell was used (Tolls et al. 2004), the Herschel/HIFI receivers (Higgins et al. 2010; Higgins 2011; Teyssier et al. 2004), and the Swedish ODIN satellite that used a gas cell filled with $\mathrm{H}_{2} \mathrm{O}$ (Frisk et al. 2003). Except for the experiment of Wehres et al. (2018a,b), most of these experimental set-ups were focused on the pre-launch characterization of receivers on board satellites and not on the possibility of using

\footnotetext{
Synergy Grant ERC-2013-SyG, G.A. 610256 (NANOCOSMOS).
} 
them for laboratory spectroscopy and chemical reactivity. A proof of concept experiment (Tanarro et al. 2018) was developed in the period 2015-2017 using a small prototype chamber placed in the beam path of a receiver covering the $42-50 \mathrm{GHz}$ band installed on the $40 \mathrm{~m}$ ARIES radio telescope at the Yebes Observatory (Spanish National Geographic Institute; hereafter IGN). These experiments showed that radioastronomical receivers can be used to perform simulations (photochemistry, cold plasma discharges) addressing chemical compositions similar to those of interstellar clouds. Thermal emission provides, in addition, partial pressures derived from the intensity and linewidth of the observed lines in the same way radio astronomers do to derive volume and column densities when observing interstellar clouds. A comparison with other techniques, such as the chirped pulse Fourier transform microwave spectroscopy, has been done by Tanarro et al. (2018).

This paper describes the final gas cell experimental set-up hereafter referred to as GAs CEll for Laboratory Astrophysics (GACELA). It has been designed to study the spectroscopy and chemistry of cold plasmas, with or without the action of ultraviolet (UV) photons, and allows us to observe the $Q$ and $W$ bands (31.5-50 and 72-116.5 GHz) simultaneously using FFTs covering the whole $Q$ band, and a selectable one-third of the $W$ band (the $W$ band is divided into three different frequency ranges selected through a motorized waveguide switch). It is the first time that an instantaneous band width of $20 \mathrm{GHz}$ in $Q$ band and $3 \times 20 \mathrm{GHz}$ in $W$ band can be used for laboratory spectroscopic experiments measuring the thermal emission of molecules. Mass spectrometry and optical spectroscopy are also performed simultaneously with the broad-band rotational spectroscopy, providing a complete set of complementary measurements. Section 2 describes the whole experimental set-up: the chamber, the vacuum system, the receivers, the spectrometers, the mass and optical spectrometers, the plasma source, the UV lamps, the data acquisition system, the calibration procedures, and the data analysis software. Section 3 is devoted to the results obtained for the different molecules used to characterize the GACELA experimental set-up. Section 4 describes the results obtained in cold plasmas with different gas compositions. Finally, Sect. 5.1 is devoted to the rotational characterization of N-ethylformamide $\left(\mathrm{CH}_{3} \mathrm{CH}_{2} \mathrm{NHCHO}\right.$; hereafter NEFA) up to $116.6 \mathrm{GHz}$ and its search in space. Appendices $\mathrm{A}-\mathrm{C}$ contain detailed descriptions of the system temperature, the data calilbration, and overviews of the full spectrum of some of the species studied in this work.

\section{The GACELA experimental set-up}

The GACELA set-up is one of the experimental developments of the NANOCOSMOS project. A prototype chamber was built at the ICMM mechanical workshop to test the concept. Results from this protoype have been published by Tanarro et al. (2018), together with detailed formulae describing the data calibration process (see also Appendix B), and how to derive physical parameters of the gas inside the chamber (partial pressures, temperatures, collisional broadening coefficients, etc.).

In this section we focus on the description of the final GACELA set-up, its subsystems (chamber, vacuum pumps, broad-band cryogenic high electron mobility transistor (HEMT) receivers, spectrometers for millimetre-wave rotational spectroscopy, and optical and mass spectrometers), the analysis of the performance of the whole system, and its capability to do novel experiments on plasma physics, photochemistry, and spectroscopy. We also address the spectroscopic characterization of several gases injected in the cell. The optical diagram of the

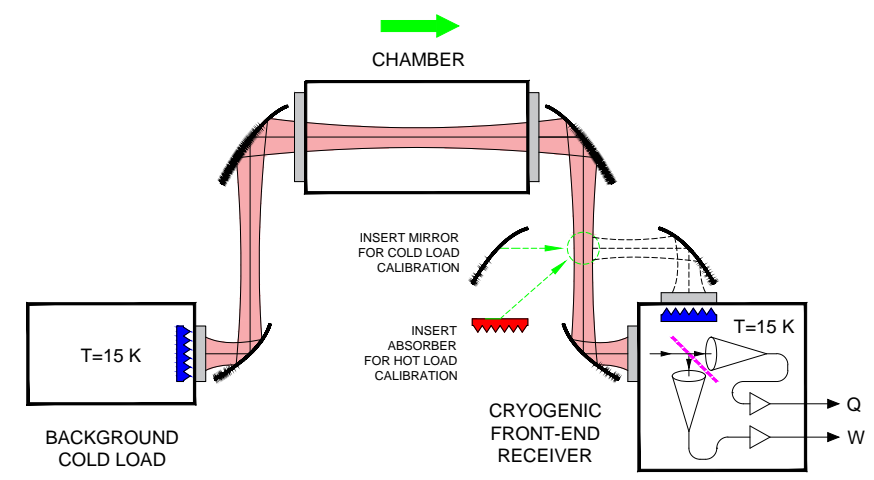

Fig. 1. Diagram of the optical path of the receivers beam in the GACELA set-up.

GACELA set-up is shown in Fig. 1. Figure A.1 shows a design of the chamber. A picture showing the coil for the radiofrequency (RF) generator, which was designed to surround the radio beam of the receivers, is shown in Fig. A.2.

During the first commissioning experiments in February 2018 we were able to detect the rotational lines of methyl cyanide $\left(\mathrm{CH}_{3} \mathrm{CN}\right)$ in a few seconds with a very high signalto-noise ratio $(\mathrm{S} / \mathrm{N})$. The lessons learned during commissioning have been crucial to improve the system performance, and for the second set of experiments that are described in Sects. 3, 4, and 5.1.

\subsection{Chamber}

The chamber consists of a stainless steel cylinder (304L) $890 \mathrm{~mm}$ in length and $490 \mathrm{~mm}$ in diameter, and with the long axis positioned horizontally. Figure A.1 shows two views (top and bottom) of the original design of the chamber (built by SEGAIN$\mathrm{VEX}^{2}$ ). The cylinder is closed by $30 \mathrm{~mm}$ thick plates that support the flanges for the microwave windows. The flanges are tilted by 9 degrees, one in the vertical plane and the other in the horizontal plane, minimizing the possible interference fringes produced by multiple reflections between the two windows (see Fig. A.1).

The flanges are of knife-edge type, size DN $250 \mathrm{CF}$, large enough (inner diameter $254 \mathrm{~mm}$ ) to accommodate the microwave Gaussian beam at the lower operating frequencies. The cell was designed with flexibility in mind, and is fitted with multiple ports (also CF type) with sizes varying from DN $40 \mathrm{CF}$ to DN $200 \mathrm{CF}$. Five ports are available on each of the four sides of the cylinder (top, bottom, left, and right; see Fig. A.1). The ports are used for the various components, including pressure/vacuum sensors, feedthroughs for electrical wiring, a manipulator for the handling of deposition substrates, RF coupling, viewports, vacuum pump connections, and gas inlets. Four more ports on the front side of the cell are provided for the coupling of ultraviolet lamps to be used in photochemistry experiments. Finally, two additional ports are fitted in the back of the cell. Although the pressures considered do not require ultrahigh vacuum (UHV) technology, we chose UHV compatible flanges for several reasons. We wanted to avoid all types of polymeric gaskets that may decompose by UV irradiation or corrosive gasses. Also, metal gaskets make the baking out processes easier when the chamber needs to be cleaned after dirty experiments. Furthermore, it helps to have less interference with the

\footnotetext{
2 Servicios Generales de Apoyo a la Investigación Experimental, Universidad Autónoma de Madrid, Ciudad Universitaria de Cantoblanco, 28049 Cantoblanco, Madrid, Spain.
} 


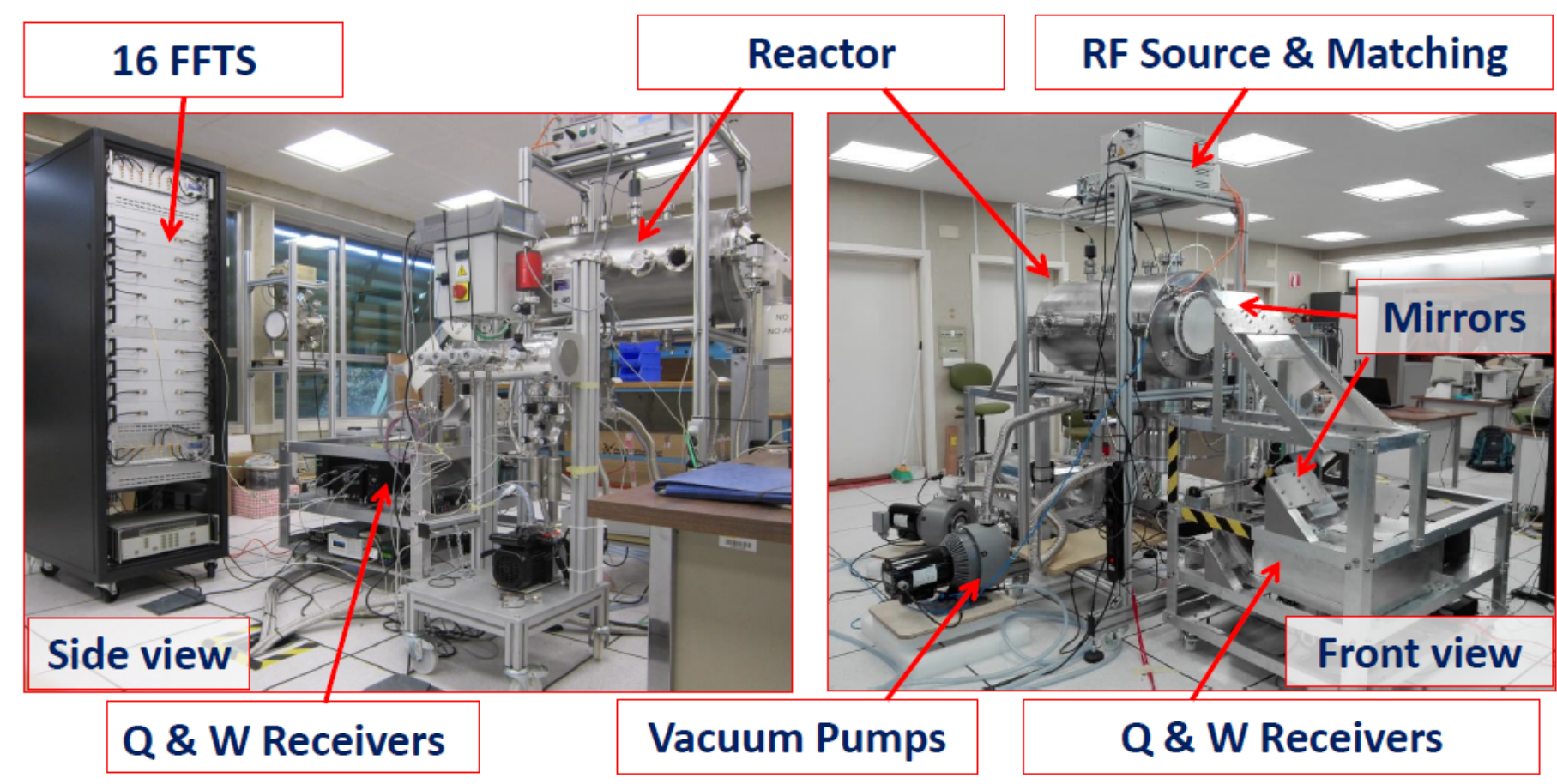

Fig. 2. Side (left) and front (right) view of the whole GACELA system. The different components are indicated by arrows. They are described in Sects. 2.1-2.4. A diagram of the optics of the system is shown in Fig. 1.

background residual gasses, which can be easily eliminated after baking out. Finally, the multiple flanges make it possible to couple the chamber to new instruments or experimental set-ups like the Stardust machine (Martínez et al. 2018).

The pumping system is composed of two scroll pumps (with $30 \mathrm{~m}^{3} \mathrm{~h}^{-1}$ pumping speed), one of which is used for rough pumping, while the other backs a turbomolecular pump (with $9501 \mathrm{~s}^{-1}$ pumping speed) connected to the bottom of the cylinder through a gate valve. Typically, a final vacuum of $\sim 2 \times 10^{-7}$ mbar is achieved.

Several gauges are available to monitor the pressure inside the cell. Pirani/Penning gauges are used to monitor the vacuum from near-atmospheric pressure down to $10^{-7}$ mbar. When gases are loaded into the chamber a capacitive type gauge (whose reading does not depend on the gas being measured) is used, covering the range $10^{-5}-10^{-1}$ mbar with a nominal precision of $0.5 \%$. For several of the experiments shown in the present work, the chamber was internally lined with a Teflon ${ }^{\mathrm{TM}}$ sheet $(2 \mathrm{~mm}$ thick) in order to decrease the molecular reactivity on the reactor walls. The gases are injected into the cell through one of the side ports to which a pre-mixing chamber was attached. This pre-mixing chamber has four inlets fitted with mass flow controllers to allow up to four different gases to be injected into the reactor. Two additional inlets can be used for liquid or solid samples. The chamber and the ducts attached to it are wrapped with heating tape in order to decrease gas adsorption and avoid the possible condensation of low vapour pressure species on the walls. A general view of the complete experimental set-up is shown in Fig. 2.

\subsection{Cryogenic front end amplifiers}

The cryogenic low-noise amplifiers (LNAs) for the $Q$ and $W$ bands of the GACELA receiver front-end are based on metamorphic GaAs millimetre-wave monolythic microwave integrated circuits (MMICs) developed by cooperation agreements of Yebes Observatory (IGN) with $\mathrm{IAF}^{3}$ and $\mathrm{UC}^{4}$ and fabricated with the $50 \mathrm{~nm}$ gate length IAF process (Leuther et al. 2009). The $Q$-band MMIC is a microstrip design incorporating four stages of $4 \times 15 \mu \mathrm{m}$ gate width HEMT devices, which can be independently biased to optimize the performance. The MMIC was mounted on a gold-plated aluminum block containing soft substrate input and output microstrip access lines and DC bias components (Fig. 3). The module can be configured either with coaxial $2.4 \mathrm{~mm}$ connectors or WR22 waveguide interfaces at input and output, although only waveguides were used in the final receiver version to minimize the loss. The $W$-band MMIC incorporates four stages of $2 \times 15 \mu \mathrm{m}$ gate width HEMT devices with independent bias and coplanar waveguide matching networks. The MMIC is housed in a gold-plated brass split-block which contains additional DC bias components (see Fig. 3). The transition between the input and output WR10 waveguides and the circuit was implemented by E-plane probes optimized for the $71-116 \mathrm{GHz}$ band and etched in a $50 \mu \mathrm{m}$ quartz substrate.

The LNAs were thoroughly characterized in the laboratory and the bias points of the different stages were optimized for an adequate compromise between noise, reflection, gain, and flatness. Special attention was paid to the stability (absence of high-frequency oscillations). Noise and gain were accurately measured using a variable temperature cryogenic waveguide load connected to the input (Malo et al. 2016), obtaining stateof-the-art average noise temperatures in the $Q$ and $W$ bands of $15 \mathrm{~K}$ and $30 \mathrm{~K}$, respectively. Noise and gain plots are shown in Fig. 4. The main results of the measurements are summarized in Table 1.

3 Fraunhofer Institut fur Angewandte Festkorperphysik Tullastrasse 72, 79108 Freiburg, Germany.

4 Universidad de Cantabria, Department of Communication Engineering, Avda. Los Castros, 39005 Santander, Spain. 

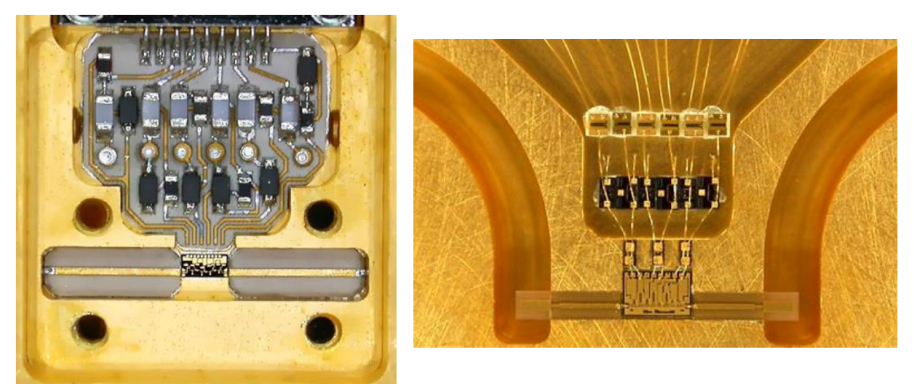

Fig. 3. Inner view of the $Q$-band (left) cryogenic amplifier module showing the MMIC, input and output microstrip lines, and bias conditioning board. MMIC size is $2 \times 1 \mathrm{~mm}$. Overall block dimensions are $40.8 \times 25.7 \times 10 \mathrm{~mm}$ including the waveguide transitions. Inner view of the $W$-band (right) cryogenic amplifier split block showing a detail of the MMIC, quartz microstrip to waveguide transitions, and DC bias protection components. MMIC size is $1.5 \times 1 \mathrm{~mm}$. Overall block dimensions are $21.5 \times 21.5 \times 19.1 \mathrm{~mm}$.

\subsection{Optics, windows, and cryostats}

The dimensions of the chamber of GACELA allow the installation of corrugated Teflon ${ }^{\mathrm{TM}}$ windows with a beam clearance diameter of $240 \mathrm{~mm}$. The windows are appropriately grooved to decrease their reflectivity at both $Q$ - and $W$-band frequencies. In the centre of the cell, the optical system provides a Gaussian half power beam-width of $54 \mathrm{~mm}$ and $40 \mathrm{~mm}$ for the $Q$-band and $W$-band receivers, respectively. The beam diameter diverges from the centre to the windows. Nevertheless, the goal of the optical design was to keep the theoretical truncation losses of the beam at each window below $0.1 \%$ (see Appendix A).

The optics were designed to allow an astronomical type ON/OFF observation and hot-cold load calibration. The optical system couples the radiation emitted by the background cold load, which interacts with the cell contents, to the feeds inside the cryogenic receiver. In the ON/OFF type observations the signals at the input of the receiver are a) the background cold load seen through the empty chamber (OFF-observation) and b) the background cold load seen trough the gases injected in the cell (ON-observation).

The coupling optics which guide the beams consists of four elliptical mirrors located on both sides of the gas-cell (see Figs. 1 and 2). With this configuration the feed beams are perfectly terminated at the background cold load. The use of shaped mirrors allows the beams to propagate through the optical circuit with estimated spill-over losses of less than $1.5 \%$ in the whole system. An additional computer controlled mobile carriage at the receiver side allows the insertion of an ambient load or an elliptical mirror which allows re-focusing the beam into an independent cold load built into the receiver cryostat. This system is used to perform the receiver calibration. The cryogenic loads are very low-reflection structures machined on ECCOSORB ${ }^{\mathrm{TM}} \mathrm{MF} 117$ magnetically loaded epoxide material, and the ambient temperature load is a piece of convoluted foam microwave absorber.

The front-end receivers and the internal cold load (ICL) are mounted in a cryostat cooled with a helium gas closed-cycle refrigerator. All components around the LNAs (polarizing grid, horn-feeds, and waveguides) are cryogenically cooled to $15 \mathrm{~K}$ in order to increase the radiometer sensitivity. Inside the receiver's cryostat, the incoming beam is split into two orthogonal polarizations using a wire grid. One polarization is directed to the $Q$-band feedhorn and the other to the $W$-band feedhorn. The background cold load (BCL) is mounted in an independent
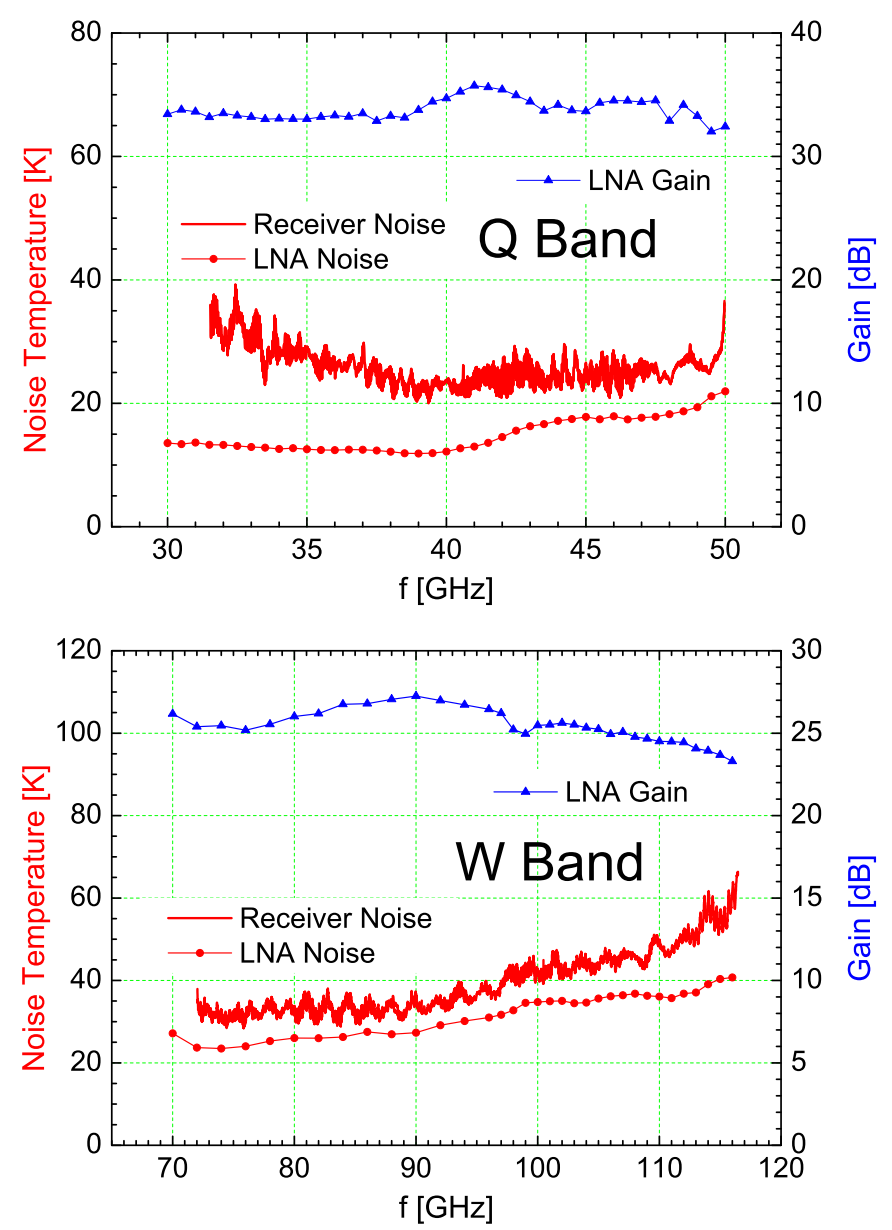

Fig. 4. Gain and noise temperature of the $Q$-band (top) and $W$-band (bottom) cryogenic LNAs at $15 \mathrm{~K}$ physical temperature compared with the noise temperature obtained in the complete receiver.

cryostat equipped with another closed-cycle refrigerator which is located on the opposite side of the chamber (see Figs. 1 and 2). Most observations are performed using it as the terminal load of the system (see Sect. 2.10).

The total receiver noise temperature including the contribution of the backend and the optics, but without the chamber is presented in Fig. 4. This figure also shows the noise temperature contribution of the cryogenic LNAs used (see Table 1). Appendix A presents more details on the determination of the contribution of the different elements of the GACELA set-up to the total system noise, $T_{\text {sys }}$, as well as the equivalent noise temperatures of the ICL and BCL. Figures A.3 and A.4 show the graphs of these temperatures as a function of frequency for $Q$ and $W$ bands, respectively.

\subsection{The intermediate frequency downconverters and the spectrometers}

The output of the receiver cryostat is connected by short waveguide sections to the ambient temperature downconverter units (Fig. 5). The $Q$-band downconverter shifts the complete $31.5-50 \mathrm{GHz}$ band to an intermediate frequency (IF) of $1-19.5 \mathrm{GHz}$ via mixing with a computer controlled synthesized local oscillator (LO). Analogously, the $W$-band downconverter accepts the complete $72-116.5 \mathrm{GHz}$ frequency range, but it processes only one of the following sub-bands at a time: a) 
Table 1. Measured performance of the cryogenic LNAs at $15 \mathrm{~K}$ physical temperature.

\begin{tabular}{lcccccc}
\hline \hline $\begin{array}{l}\text { Amplifier } \\
\text { serial number }\end{array}$ & $\begin{array}{c}\text { Band } \\
(\mathrm{GHz})\end{array}$ & $\begin{array}{c}T_{\mathrm{n}}(\mathrm{av}) \\
(\mathrm{K})\end{array}$ & $\begin{array}{c}\mathrm{G}(\mathrm{av} .) \\
(\mathrm{dB})\end{array}$ & $\begin{array}{c}\mathrm{IRL}(80 \%) \\
(\mathrm{dB})\end{array}$ & $\begin{array}{c}\text { ORL(80\%) } \\
(\mathrm{dB})\end{array}$ & $\begin{array}{c}P_{\text {diss }} \\
(\mathrm{mW})\end{array}$ \\
\hline YMWN 2006 & $72-116$ & 30.7 & 25.6 & -6.3 & -13.7 & 23.2 \\
YMQ 2002 & $31-50$ & 15.1 & 33.8 & -5.1 & -12.0 & 14.8 \\
\hline
\end{tabular}

Notes. The values of noise temperature $\left(T_{\mathrm{n}}\right)$ and gain $(\mathrm{G})$ are the average value in the band, while the values of the input and output reflection losses (IRL, ORL) are the worst case in $80 \%$ of the band. $P_{\text {diss }}$ is the total heat power dissipation at the optimum bias value.

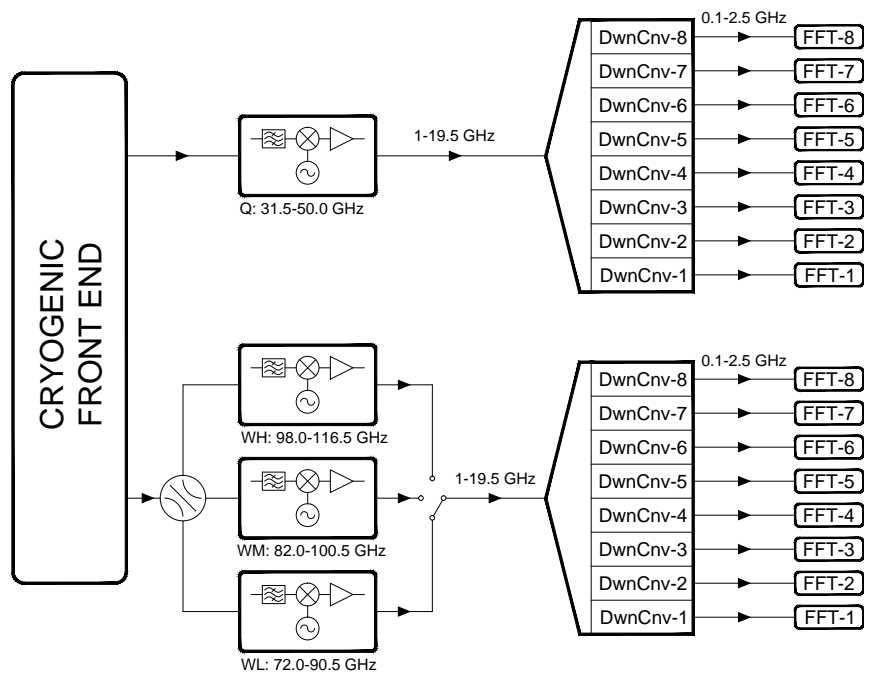

Fig. 5. Block diagram of the receiver system.

$72-90.5 \mathrm{GHz}$ (WL), b) $82-100.5 \mathrm{GHz}$ (WM), or c) 98$116.5 \mathrm{GHz}(\mathrm{WH})$. A remotely controlled waveguide switch selects the specific sub-band to be converted to the $1-19.5 \mathrm{GHz}$ IF. Image frequencies of the mixing process are effectively rejected by band pass filters. The frequency of the synthesizers used for LOs can be varied under computer control to make frequency switching type observations possible. A view of the implementation of the $W$-band unit is shown in Fig. 6.

The $\mathrm{Q}$ and $\mathrm{W}$ downconverter blocks provide an overall gain of $30-40 \mathrm{~dB}$ with noise temperatures in the $500-1500 \mathrm{~K}$ range. The $1-19.5 \mathrm{GHz}$ IF signals from the $Q$ and $W$ bands are sent through low-loss coaxial cables to distribution units for further processing by an array of eight additional downconverters (see Fig. 5). Each one shifts a specific $2.5 \mathrm{GHz}$ slot of the $1-19.5 \mathrm{GHz}$ input range to baseband, so the FFT spectrometers can be fed with a suitably conditioned signal. The frequencies of the LOs for the second down conversion are fixed, and the gain of each unit is adjustable by $1 \mathrm{~dB}$ steps to optimize the power level at the digitizer input. The spectrum of the baseband signal is computed in an FFT module containing an analogue-to-digital converter and a field-programmable gate array for real time signal processing. Each FFT module accepts a $0.1-2.5 \mathrm{GHz}$ input signal and provides a spectrum with 65536 points which corresponds to $38.14 \mathrm{KHz}$ of frequency resolution.

The FFT spectrometers were provided by Radiometer Physics, GmbH. Figure 2 shows the rack cabinet with the distribution modules (central position), the IF downconverters, and the two FFTs blocks (at the top and bottom positions). The arrangement of the modules is optimized to reduce the length of the connecting cables and to allow an adequate air circulation for cooling. We note that due to the particular system architecture, the $Q$ band can always be observed together with one of the

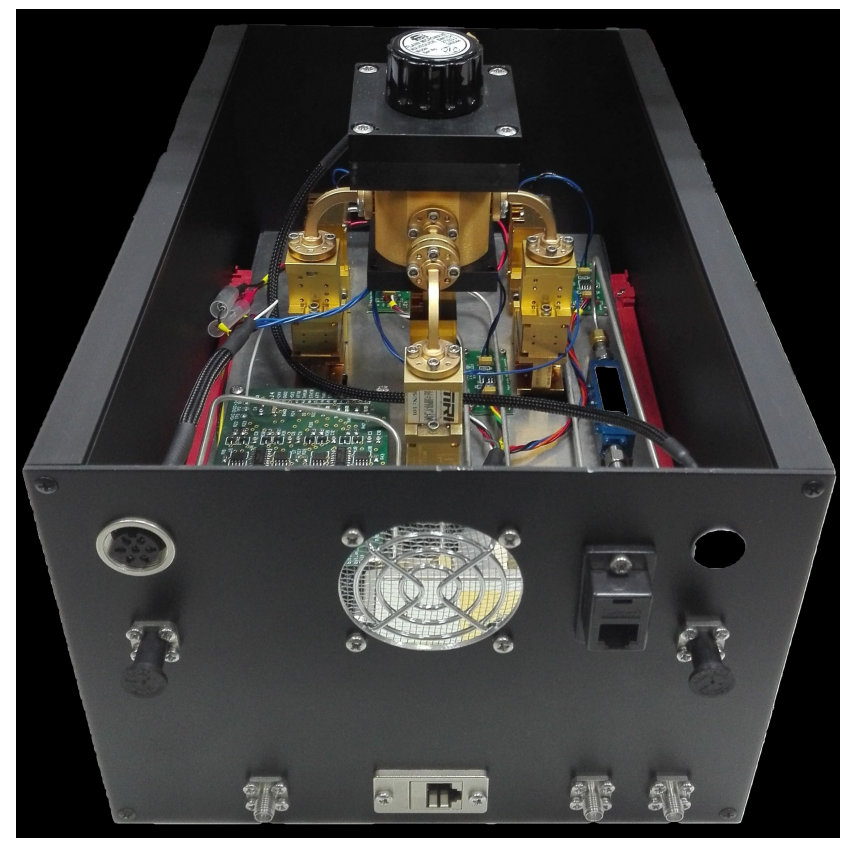

Fig. 6. $W$-band downconverter for the GACELA receiver. The remotely controlled waveguide switch allowing the selection of one of three $W$ sub-bands is clearly seen at the middle of the box.

three selected $W$ sub-bands. Hence, for the complete frequency coverage of the $W$ band, the $Q$ band will have three times more observing time than any of the three $\mathrm{W}$ sub-bands. The adopted blanking time to switch from one $W$ sub-band to another is one second. All the LOs, frequency synthesizers, and clocks of the FFT modules are phase locked to a $5 \mathrm{MHz}$ central frequency reference standard obtained from the Yebes Observatory hydrogen maser to ensure the accuracy of the frequency scale.

\subsection{Control software}

The radio receivers and backends, together with the auxiliary equipment are controlled from a central software program which mimics the one used at the $40 \mathrm{~m}$ radio telescope at the Yebes Observatory. The software performs two kind of operations: the control and configuration of the receiving system and data acquisition, and a first processing of the spectroscopic data and its storage. The control is done from a Linux PC using the ALMA Common Software (ACS) as the basic element, which allows the distribution of tasks and coordination of the different devices involved in the operation. This infrastructure uses CORBA as communication mechanism, implements a component-container paradigm and allows the use of different programming languages (C++, Java, and Python) and its seamless interconnection. Most of the devices are connected to a private local area network at 
1 Gbps, although some of them are read and configured using serial and GPIB connections. In these latter cases ethernet converters are used to facilitate the operations.

The control of the mass and optical spectrometers, which are used as ancillary equipment for the detection of molecular species, are not integrated in the ACS. They are operated in an independent way from their own control and monitoring software (see Sects. 2.8 and 2.9).

The interface with the scientist is a command line based on IPython; it is fully programmable, which allows complex operations like repetitive macros, but retains a great simplicity in its usage. The basic supported operations are the control of the receiver: selection of frequency sub-bands in the $72-116.5 \mathrm{GHz}$ band, the attenuation of the intermediate frequency (IF) signal, the monitoring and tuning of the LOs, the calibration system (which uses hot and cold loads), the configuration of the integration time, phase composition and spectral resolution of the FFTs backends. In addition the control of the RF generator for cold plasmas, the ON/OFF switching of the UV lamps, and the readout of multiple pressure sensors of the system are also operated through this interface.

A graphical interface panel written in Python-Qt allows us to visualize in real time the current status of the subsystems (receivers, loads, backends, and sensors) and the running scan. This panel also allows us to set scan options like the duration of the phases and their order, scan type, and number of repetitions. The time evolution of some sensors readings, like pressure and temperature, can be plotted in real time.

The current system generates 16 spectra with 65536 channels each coming from 16 different FFTs which are read continuously and integrated to reduce the storage volume and the processing load. Integration times can be set from $100 \mathrm{~ms}$ to $5 \mathrm{~s}$. The FFTs phases allow spatial observations (ON phase with the chamber full of gas and OFF phase with the chamber empty of gas) and frequency switching (FS) observations in which the LO swaps its frequency by tens of $\mathrm{MHz}$ (usually $25-50 \mathrm{MHz}$ ) typically every two seconds (see Sect. 2.10). The control software allows a correct synchronization between the LO and the data acquisition. This synchronization procedure optimizes the integration time, but relies on a uniform behaviour of the IF along the frequency and usually requires a post-fit of the spectrum baseline.

The data are generated in CLASS format, a program from the GILDAS package ${ }^{5}$ developed at the Institut de Radioastronomie Millimétrique (IRAM) and used in spectroscopic observations at many radio telescopes around the world. Part of the header for this spectrum, which is not useful for laboratory experiments, has been adapted to store information from the different sensors that monitor the conditions of the experiment, for example, pressure and temperature at the receiver, at the chamber, and the cold calibration load. All data collected by the system can be fully processed in CLASS (data addition, baseline removal, and fitting of Gaussian line profiles, among other functionalities).

All data are stored in files and sorted in folders conveniently named and tagged to allow simple operations and easy identification. All metadata and operations are stored in a MySQL database to allow fast and simple searches.

The system permits automatic overnight observations and integration times as long as $6.05 \times 10^{5} \mathrm{~s}$, which were reached during a stand-alone observation that lasted one week.

\footnotetext{
http://www.iram. fr/IRAMFR/GILDAS
}

\subsection{Plasma source}

In order to study chemical processes, the cell is fitted with an internal coil that can inductively couple a plasma inside the chamber. It is formed by four turns of $6 \mathrm{~mm}$ diameter copper tubing, positioned in the centre of the chamber, which avoids any vignetting of the detector field of view. The diameter of the windings is $250 \mathrm{~mm}$, and they are spaced by $\sim 5 \mathrm{~cm}$. One end of the coil is connected to the impedance matching unit of the RF generator, which can provide up to $300 \mathrm{~W}$ RF power at $13.56 \mathrm{MHz}$, while the other end is in electrical contact with the cell body and grounded. The coil is wrapped with Teflon ${ }^{\mathrm{TM}}$ tape $(50 \mu \mathrm{m}$ thick) in order to decrease the reactivity of some gases with the copper surface. The coil and its position inside the cell are shown in Fig. A.2.

\subsection{Ultraviolet lamps}

In order to study ultraviolet (UV) induced processes, the cell is also fitted with four UV lamps that are placed symmetrically along its inner wall. The lamps are harboured by fused silica tube-shaped sheaths. One end of the sheath is open and bonded to a CF40 flange that attaches to the front plate of the chamber, while the inner end of the sheath is closed. In this way the lamps are operated at ambient pressure and can be replaced without breaking the vacuum inside the cell. A flow of nitrogen purges the space between the lamps and their sheaths, removing atmospheric $\mathrm{O}_{2}$ and $\mathrm{H}_{2} \mathrm{O}$ (which, together with the formed $\mathrm{O}_{3}$, would absorb part of the UV radiation), and also providing some cooling to the lamps. The lamps themselves are of lowpressure mercury-arc type, with a total length of $793 \mathrm{~mm}$ (the discharge extends for $713 \mathrm{~mm}$ ) and a diameter of $19 \mathrm{~mm}$. They have a total output power of $13.5 \mathrm{~W}$ in the UV-C region according to the manufacturer, with $\sim 11.7 \mathrm{~W}$ in the $254 \mathrm{~nm}$ line of $\mathrm{Hg}$ and $\sim 1.8 \mathrm{~W}$ in the $185 \mathrm{~nm}$ line. Figure A. 2 shows the interior of the chamber with the UV lamps, the RF coil, and the Teflon ${ }^{\mathrm{TM}}$ lining.

The UV lamps were successfully tested by using a flowing mixture of $\mathrm{C}_{2} \mathrm{H}_{2}$ and $\mathrm{NH}_{3}$. The experiment was also performed in static conditions. In both cases the main products were $\mathrm{HCN}, \mathrm{HC}_{3} \mathrm{~N}, \mathrm{HC}_{5} \mathrm{~N}$, and other compounds detected by mass spectroscopy. These results will be published elsewhere.

\subsection{Mass spectrometer}

In order to have more information on the composition and abundances of chemical species inside the cell, a quadrupole mass spectrometer is attached to one of the ports. The spectrometer is a residual gas analyser (Hiden, HAL 201 RGA, with a dual Faraday/Channeltron Electron Multiplier detector) and can measure masses up to $200 \mathrm{u}$. It is operated at pressures below $10^{-7}$ mbar with a dedicated vacuum system (turbomolecular plus scroll pumps), and it is connected to the gas cell via an electronically controlled needle valve. In Sect. 4.3 we show a mass spectrum taken when an RF plasma was induced in a flowing mixture of methane $\left(\mathrm{CH}_{4}\right)$ and nitrogen $\left(\mathrm{N}_{2}\right)$ inside the chamber.

\subsection{Optical spectrometer}

The plasma optical emission is collected through a fused silica window placed in a DN $60 \mathrm{CF}$ side flange of the chamber by means of a fused silica fiber and a $193 \mathrm{~mm}$ focal length, motorized Czerny-Turner spectrograph (Andor, Shamrock SR-193-i-A), equipped with a CCD camera (iDus DU420A-BVF). Two 
J. Cernicharo et al.: Broad-band rotational spectroscopy for laboratory astrophysics
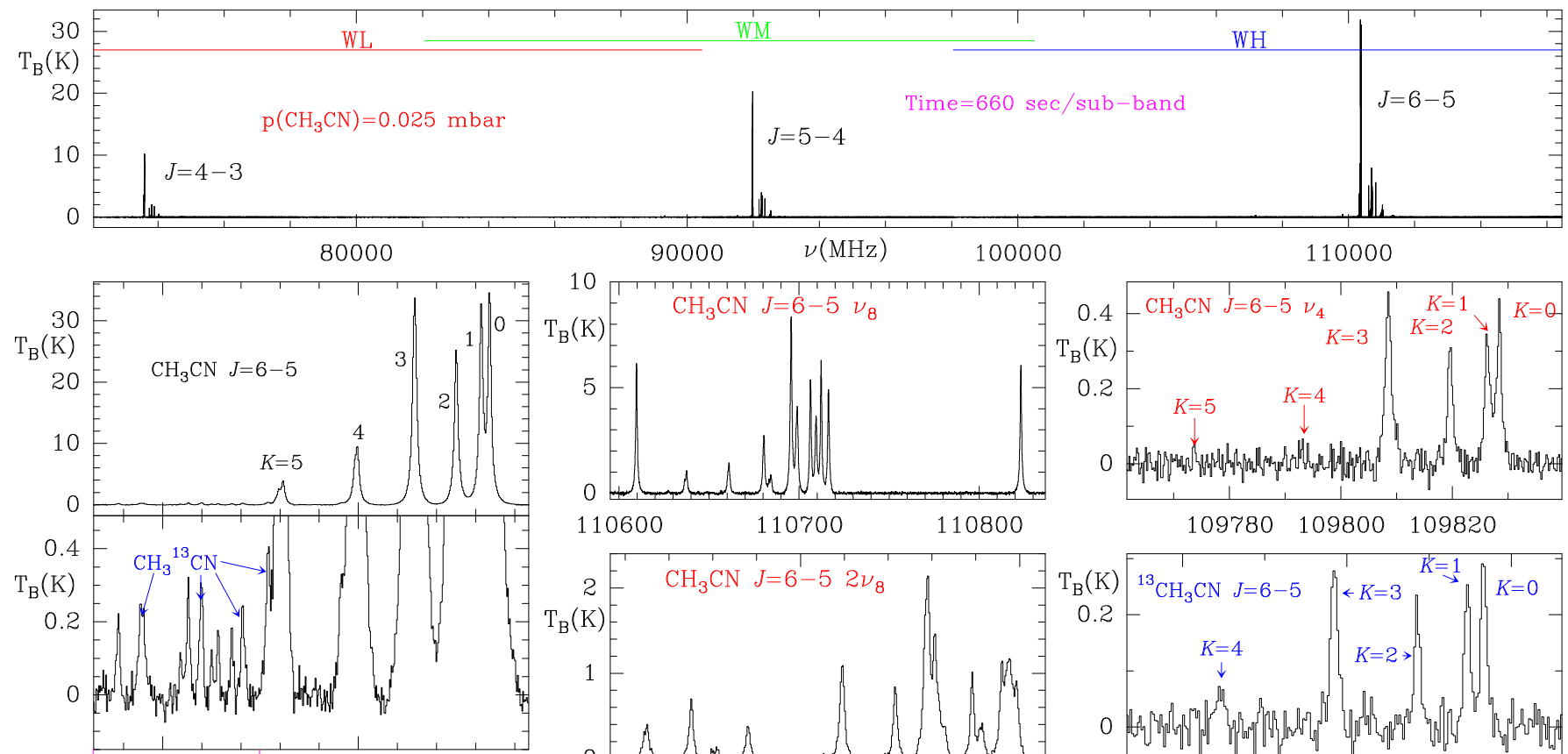

$109780109800 \quad 109820$
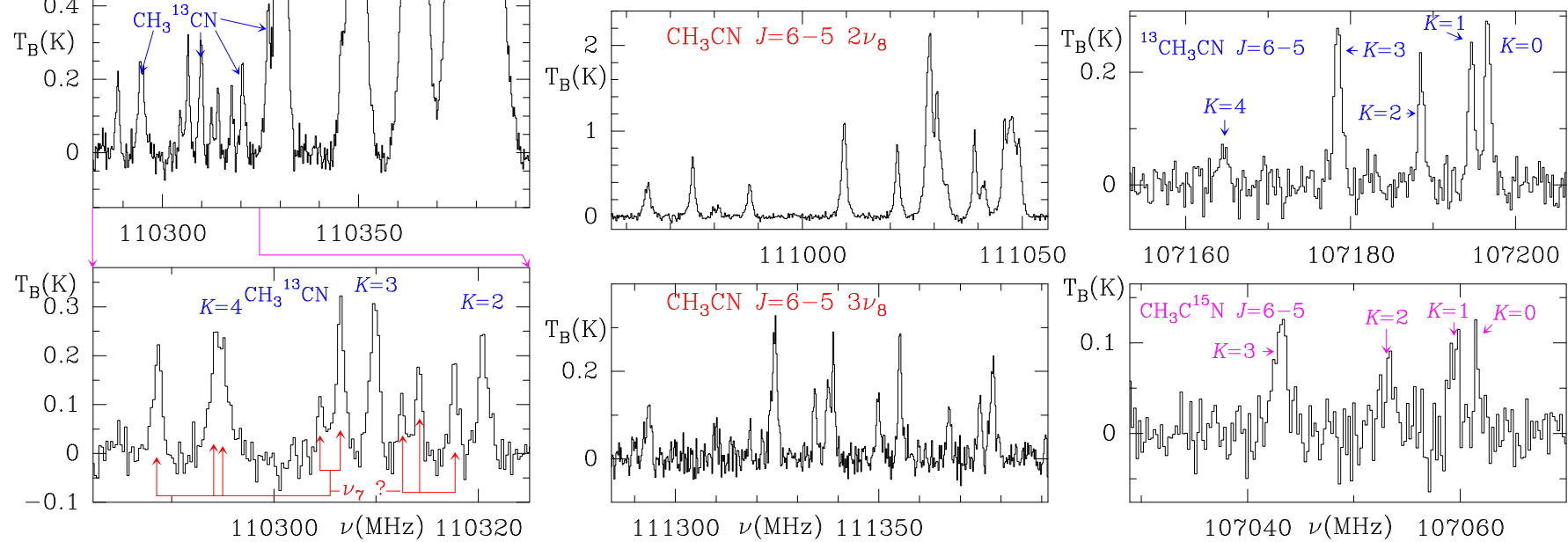

$111300 \nu(\mathrm{MHz}) 111350$

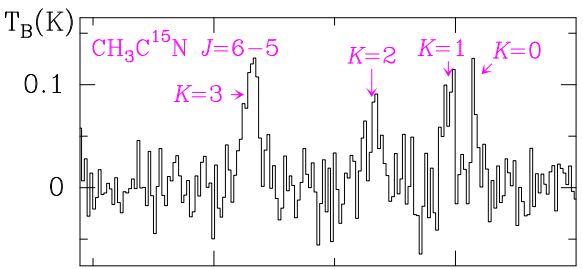

$107040 \nu(\mathrm{MHz}) \quad 107060$

Fig. 7. Observation of $\mathrm{CH}_{3} \mathrm{CN}$ in flow at a total pressure of $25 \times 10^{-3}$ mbar with GACELA using a load switching observing procedure. Upper panel: whole $72-116 \mathrm{GHz}$ range resulting from the observation of the three sub-bands of the $W$-band receiver. The integration time is 11 min per sub-band. Left column: zoom of the $J=6-5$ transition of $\mathrm{CH}_{3} \mathrm{CN}$ (upper panel), $\mathrm{CH}_{3}^{13} \mathrm{CN}$ (middle panel), and again $\mathrm{CH}_{3}^{13} \mathrm{CN}_{\text {together with }}$ probably the $v_{7}$ or $v_{4}+v_{8}$ mode of $\mathrm{CH}_{3} \mathrm{CN}$ (bottom panel). Three panels in the middle column: $J=6-5$ transition of $\mathrm{CH}_{3} \mathrm{CN}$ in the bending mode $v_{8}$ (upper panel), and its overtones at $2 v_{8}$ (middle panel) and $3 v_{8}$ (bottom panel). Three panels in the right column: $J=6 \rightarrow 5$ transition of the $v_{4}$ mode of $\mathrm{CH}_{3} \mathrm{CN}$ (upper panel), the same transition of the ground state of ${ }^{13} \mathrm{CH}_{3} \mathrm{CN}$ (middle panel), and of $\mathrm{CH}_{3} \mathrm{C}^{15} \mathrm{~N}$ (bottom panel). All the singly substituted isotopologues of methyl cyanide are in natural abundance. Intensities are expressed in $\mathrm{K}$ and frequencies in MHz. The kinetic temperature of the gases inside chamber is $297 \mathrm{~K}$ in these experiments. The difference between the observed frequencies and those measured in other laboratories, or predicted from accurate rotational constants, are less than $5 \mathrm{kHz}$ for signals above $0.2 \mathrm{~K}$ and $\sim 5-20 \mathrm{kHz}$ for the weakest features.

diffraction gratings with 1200 grooves $\mathrm{mm}^{-1}$ and 1800 grooves $\mathrm{mm}^{-1}$ provide spectral ranges of $300-1200 \mathrm{~nm}$ and $200-950 \mathrm{~nm}$, respectively, and nominal spectral resolutions of $0.22 \mathrm{~nm}$ and $0.15 \mathrm{~nm}$, respectively (for a $26 \mu \mathrm{m}$ slit width). The relative spectral efficiency of the whole system was determined with a tungsten lamp calibrated in the optical metrology laboratories of the Institute of Optics of CSIC ${ }^{6}$. In Sect. 4.3 we show the optical spectrum emitted by a $\mathrm{CH}_{4}+\mathrm{N}_{2}$ plasma, acquired with the 1800 grooves $\mathrm{mm}^{-1}$ grating.

\subsection{Observing procedures}

The best observing procedure for removing the instrumental response consists in performing a filled chamber observation, ON phase, minus an empty chamber observation, OFF phase. Unfortunately, switching between the ON and OFF phases takes a long time because of the residence time of the gases in the chamber and the time needed to refill the chamber at the same

\footnotetext{
6 Institute of Optics (IO.CSIC), Department of Optical Radiation Measurement, c/ Serrano 144, 28006 Madrid (Spain).
}

initial pressure. Hence, two alternative observing modes have been tested providing reasonable baselines for all tested pressures $\left(10^{-6}-40 \times 10^{-3}\right.$ mbar $)$.

The first procedure consists in taking the ICL as OFF observation. Obviously, the ripples of the ICL are different from those of the chamber plus the BCL. This problem has been solved by observing, at the beginning of each experiment, a reference spectrum of the ICL and the BCL through the empty chamber. The ratio of the two cold loads, $\mathrm{R}=\mathrm{BCL} / \mathrm{ICL}$, was used during observations to generate an OFF for each ON scan. For each scan $i$ the $\mathrm{OFF}_{i}$ was computed as $R \times \mathrm{ICL}_{i}$. The obtained baselines are rather flat (see Fig. 7), but after 10-15 min it was necessary to take a new reference spectrum of the ICL and of the empty chamber plus BCL. Moreover, the switch between the positions corresponding to the chamber and the ICL takes several seconds as the movable mirror has to move around $40 \mathrm{~cm}$ between the two optical paths. The frequent pump/filling cycles plus the dead time needed for this displacement render the observing procedure inefficient.

The second procedure is frequency switching (FS), typically with a throw of $25-50 \mathrm{MHz}$. The baselines show a considerable 


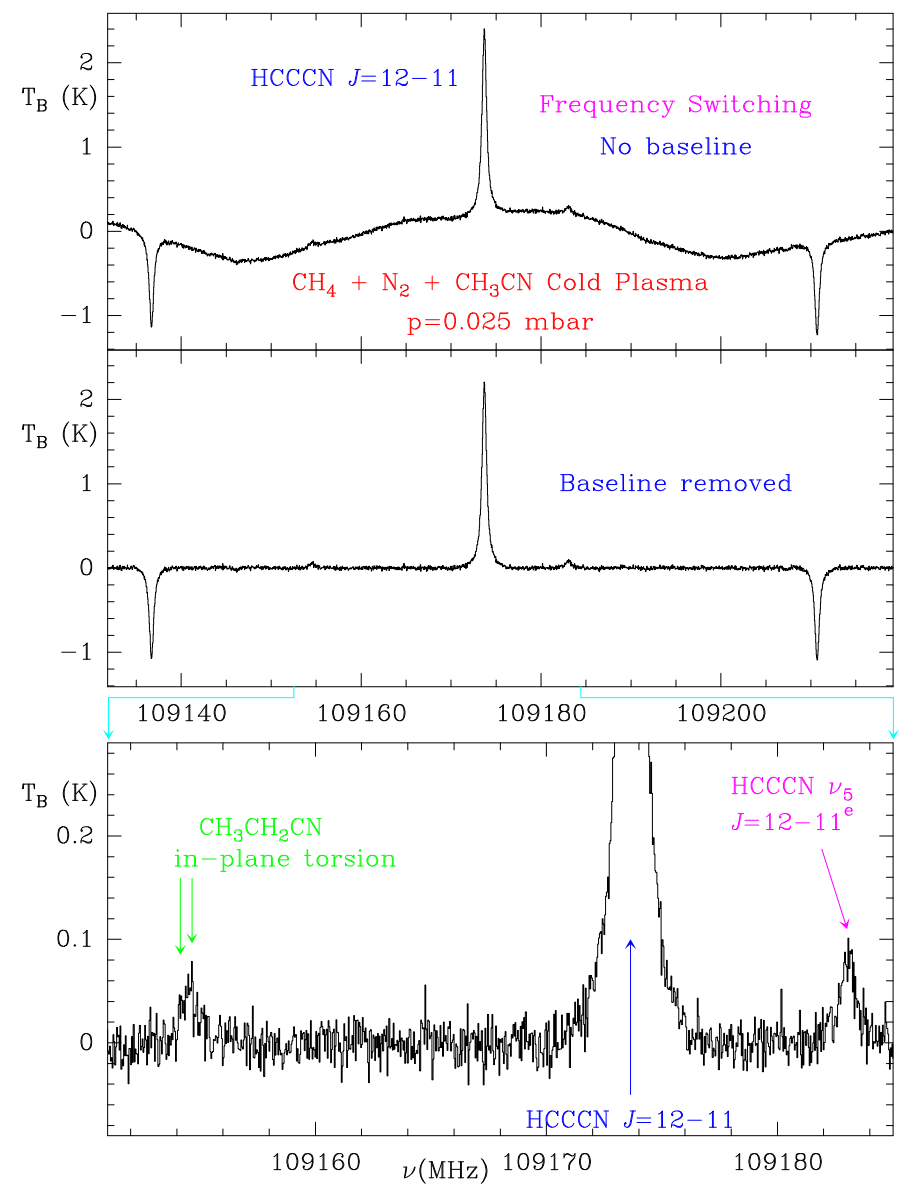

Fig. 8. Cold plasma of $\mathrm{CH}_{4}, \mathrm{~N}_{2}$, and $\mathrm{CH}_{3} \mathrm{CN}$ observed in frequency switching mode with a frequency throw of $37 \mathrm{MHz}$. The data have been folded. Upper panel: observed spectrum around $\mathrm{HC}_{3} \mathrm{~N} J=12 \rightarrow 11$ (produced in the plasma) without removing the baseline. Middle panel: resulting spectrum when a polynomial baseline is removed. The bottom spectrum is magnified in frequency and intensity to show the weak lines that were detected from $\mathrm{HC}_{3} \mathrm{~N} v_{5}\left(E_{\text {up }} \simeq 1000 \mathrm{~K}\right)$ and the in-plane torsion of $\mathrm{CH}_{3} \mathrm{CH}_{2} \mathrm{CN}$ (produced in the plasma, $E_{\text {up }} \simeq 320 \mathrm{~K}$ ). The integration time for this observation is $100 \mathrm{~s}$.

number of ripples, but the lowest frequency ripple has a period of $40 \mathrm{MHz}$, which permits us to derive accurate line profiles and intensities for lines of up to $\sim 2 \mathrm{MHz}$ full width at half maximum (FWHM). This is the recommended observing procedure as the lines are observed twice and the noise gets improved by a factor of the square root of two. The frequency switch is fast enough (a few milliseconds) to minimize blanking times. Figure 8 shows the observed spectra in FS mode around the frequency of the $J=$ $12 \rightarrow 11$ line of $\mathrm{HC}_{3} \mathrm{~N}$ formed in a plasma of $\mathrm{CH}_{4} / \mathrm{N}_{2} / \mathrm{CH}_{3} \mathrm{CN}$ at a total pressure of $25 \times 10^{-3}$ mbar. The ripples with frequencies above $40 \mathrm{MHz}$ can be easily eliminated by applying a FFT to each spectrum and removing the corresponding time domain, or by a polynomial baseline removal.

\section{Sensitivity and spectroscopic measurements with GACELA}

\subsection{Integration time and sensitivity}

In order to check the longest integration time for which our system $\mathrm{S} / \mathrm{N}$ performs correctly (i.e. the noise decreases with the square root of the integration time), as a first experiment, we

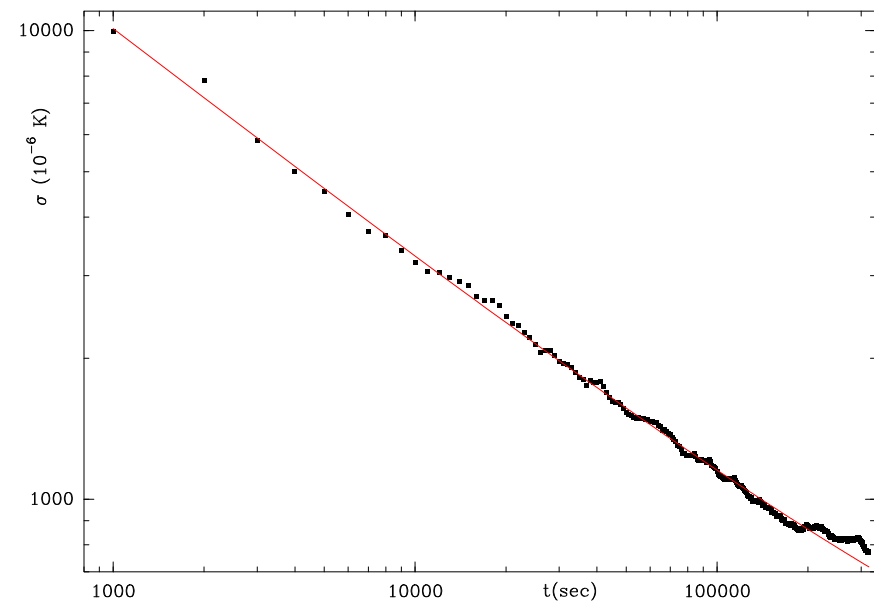

Fig. 9. Noise as a function of time in sub-band WM for a frequency domain of $40 \mathrm{MHz}$ around $88630 \mathrm{MHz}$. Each individual point represents a $1000 \mathrm{~s}$ increase in observing time.

performed an observation with very long integration time (3 days per W sub-band), with a frequency switch of $37 \mathrm{MHz}$ and with the cell at $10^{-7}$ mbar. The theoretical noise, $\sigma$, for a frequency switching observation (after frequency folding) is given by

$\sigma=\frac{\sqrt{2} T_{\mathrm{sys}}}{\sqrt{\Delta v t}}$,

and for an observation in ON/OFF mode by

$\sigma=\frac{2 T_{\text {sys }}}{\sqrt{\Delta v t}}$

where $\sigma$ and the system temperature, $T_{\text {sys }}$, are in $\mathrm{K} ; t$ is the total integration time (in seconds); and $\Delta v$ is the spectral resolution (in $\mathrm{Hz}$ ).

Figure 9 shows the computed noise over a band of $40 \mathrm{MHz}$ around the frequency of the $J=1 \rightarrow 0$ line of $\mathrm{HCN}$; we note that residual gases do not emit at these frequencies and that the chamber was completely clean during the experiment. The noise, $\sigma$, decreases as the square root of time up to an observing time as long as $2 \times 10^{5} \mathrm{~s}$. For longer times, $\sigma$ decreases more slowly than predicted by theory for the selected frequency range. In the three sub-bands of the $W$ band the behaviour of $\sigma$ with time is similar to that shown in Fig. 9. Some frequency domains in $W$ band, typically $50-200 \mathrm{MHz}$ wide, show a poorer behaviour, with a longer integration time (following the square root dependence with time) of $1-1.5 \times 10^{5} \mathrm{~s}$. In the $Q$ band the Teflon ${ }^{\mathrm{TM}}$ windows are not as well matched as in the $W$ band, and there are frequency domains where the longest integration time is $3-6 \times 10^{4} \mathrm{~s}$. Taking into account the system temperatures (see Figs. A.3 and A.4), these observing times imply a correct behaviour of the system down to $0.7-1.5 \mathrm{mK}$ over the $W$ band, and $1-4 \mathrm{mK}$ in the $Q$ band.

The WiFi signal of the Observatory is observed as a broad feature, $40 \mathrm{MHz}$ wide, in two FFTs of the $W$ sub-bands and one of the $Q$ band. Some spurious narrow features (1-3 channels) were also found in the $Q$ and $W$ bands. These features are easily identified and blanked (or interpolated) during data analysis.

\subsection{Frequency measurements}

In order to test the performance of the GACELA set-up as tool for molecular aspectroscopy, we used $\mathrm{CH}_{3} \mathrm{CN}$, OCS, and $\mathrm{SO}_{2}$ to carry out different experiments. In particular, we analysed 


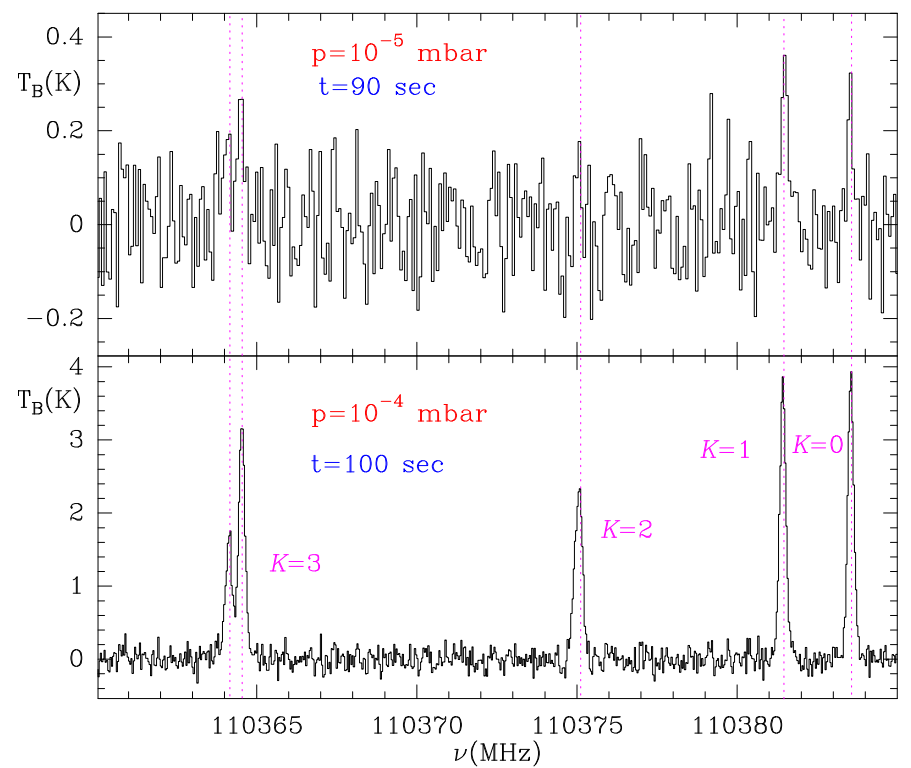

Fig. 10. Lines from the $J=6 \rightarrow 5$ rotational transition of $\mathrm{CH}_{3} \mathrm{CN}$ observed at a pressure of $10^{-5} \mathrm{mbar}$ (top panel) and $10^{-4} \mathrm{mbar}$ (bottom panel) with integration times of 90 and $100 \mathrm{~s}$, respectively. The observing mode is ON/OFF. Intensity is expressed in $\mathrm{K}$ and frequency in $\mathrm{MHz}$. The intensities are proportional to pressure as collisional broadening is marginal at these pressures. The hyperfine structure of the $K=3$ component is clearly seen in the bottom panel. The $K=2$ component exhibits an emerging left shoulder due to the hyperfine splitting of the line.

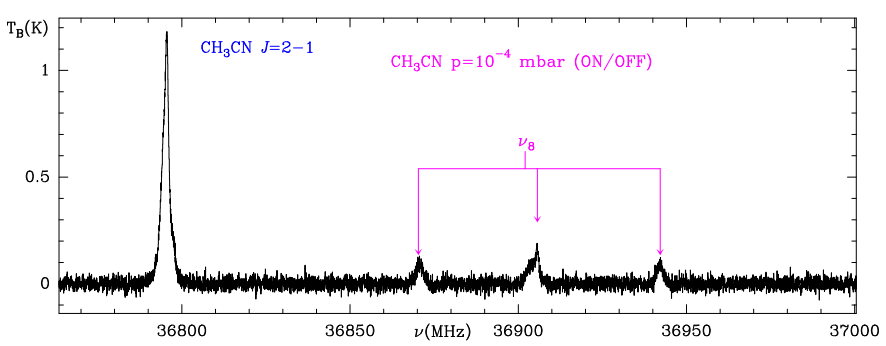

Fig. 11. The $J=2 \rightarrow 1$ transition of $\mathrm{CH}_{3} \mathrm{CN}$ in the ground state and in the $v_{8}$ mode. Integration time is $33 \mathrm{~min}$, i.e. three times that of the $W$ band. The $Q$ band is always observed independently of the selected sub-band of the $W$-band receiver.

the quality of the baseline, the appropriate working pressures for each gas to have reasonable linewidths, and the accuracy that can be reached in the measurement of the frequencies of the rotational transitions of gases inside the chamber. As commented above (see Sect. 2.10), the sharpest ripples of our detection system have a period of $\sim 40 \mathrm{MHz}$. Hence, the instrumental frequency response could affect lines with a FWHM larger than $\sim 2 \mathrm{MHz}$. Methyl cyanide $\left(\mathrm{CH}_{3} \mathrm{CN}\right)$ was the first molecule we observed during the commissioning of GACELA. This molecule exhibits a large self-broadening with pressure, of the order of $50 \mathrm{MHz}$ mbar $^{-1}$. To limit the possible problems with the ripples we tested several pressures and concluded that the best operating conditions were obtained for $P=0.02-0.04$ mbar. Figure 7 shows the $W$ band spectrum obtained after a $660 \mathrm{~s}$ integration time per $W$ sub-band at a $\mathrm{CH}_{3} \mathrm{CN}$ pressure of $25 \times 10^{-3} \mathrm{mbar}$. All the singly substituted isotopologues are detected $\left(\mathrm{CH}_{3}^{13} \mathrm{CN}\right.$, ${ }^{13} \mathrm{CH}_{3} \mathrm{CN}$, and $\left.\mathrm{CH}_{3} \mathrm{C}^{15} \mathrm{~N}\right)$, together with the vibrational states $v_{4}, v_{8}, 2 v_{8}, 3 v_{8}$, and probably $v_{7}$ and/or $v_{4}+v_{8}$.

Several pressures were tested. At $10^{-5}$ mbar we expected to find a featureless spectrum. However, the $K=0,1$ lines of the

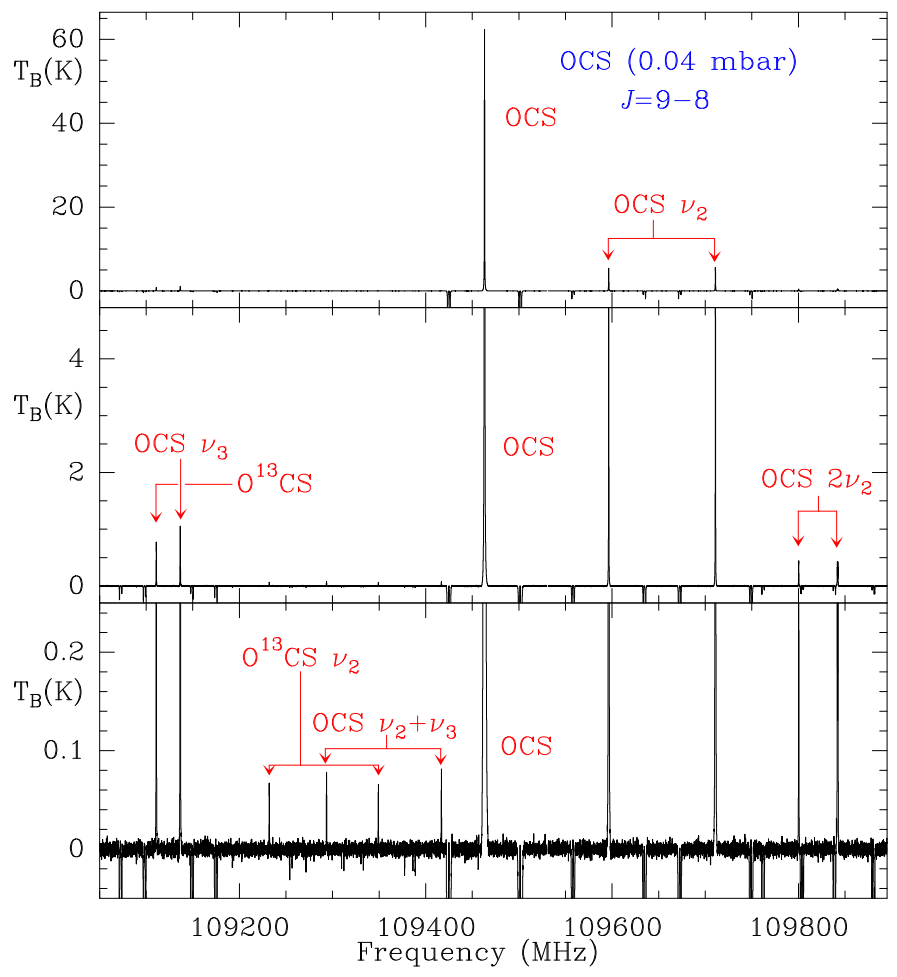

Fig. 12. Observation of OCS at a total pressure of $0.04 \mathrm{mbar}$ in the chamber using a frequency switching observing procedure. The different panels show the $109050-109895 \mathrm{MHz}$ frequency domain within the WH sub-band, but with different intensity scale limits (in K). Individual features are identified. The total observing time in this frequency range is $2.2 \mathrm{~h}$. The negative features seen in the different panels are produced by the folding of the frequency switched data and appear at $\pm 37 \mathrm{MHz}$ of each rotational transition (see also Figs. C.1 and C.2).

$J=6 \rightarrow 5$ transition of $\mathrm{CH}_{3} \mathrm{CN}$ were detected at $4.5 \sigma$ in $90 \mathrm{~s}$ (see Fig. 10 upper panel) and the $K=3$ with its hyperfine structure at $3 \sigma$. The lines become prominent at $10^{-4} \mathrm{mbar}$ (see Fig. 10 bottom panel; $100 \mathrm{~s}$ observing time), and very strong at $25 \times 10^{-3}$ mbar (see Fig. 7). The observing mode was ON/OFF in all cases. For $10^{-4}$ mbar the lines of the $v_{8}$ mode appear clearly in $100 \mathrm{~s}$. We note that at $10^{-5}$ mbar the density of $\mathrm{CH}_{3} \mathrm{CN}$ at $300 \mathrm{~K}$ is $\sim 3 \times 10^{11} \mathrm{~cm}^{-3}$, and that the column density in the cell is $\sim 2.7 \times 10^{13} \mathrm{~cm}^{-2}$, i.e. three to four orders of magnitude below the column density of this molecule in Orion (Bell et al. 2014).

The $J=2 \rightarrow 1$ transition of $\mathrm{CH}_{3} \mathrm{CN}$ falls in the $Q$ band. The lines are expected to have a much weaker intensity (due to the lower Einstein coefficients) when compared with the lines in the $W$ band $(J=4 \rightarrow 3,5 \rightarrow 4$, and $6 \rightarrow 5$ ). Nevertheless, the largest integration time of this band, a factor of three compared with the whole $W$ band (the $Q$ band is always observed together with the WL, WM, or WH sub-band), permits us to detect the lines of the ground state and the $v_{8}$ mode (at $362 \mathrm{~cm}^{-1}$ or $521 \mathrm{~K}$ ) (see Fig. 11) during an integration of $33 \mathrm{~min}$. The line broadening seen in Fig. 11 is due to the different hyperfine components of each transition.

The second molecule used during our tests was OCS. The pressure broadening for this molecule is less severe than that for methyl cyanide (Koshelev \& Tetryakov 2009). Several pressures were tested and we selected 0.04 mbar. The whole data set in the $Q$ and $W$ bands is shown in Fig. C.1. A polynomial baseline was removed by pieces $30 \mathrm{MHz}$ wide in the $Q$ and $W$ bands. Figure 12 shows the observed features in a $1 \mathrm{GHz}$ domain around the $J=9 \rightarrow 8$ transition of OCS, which correspond mostly to 

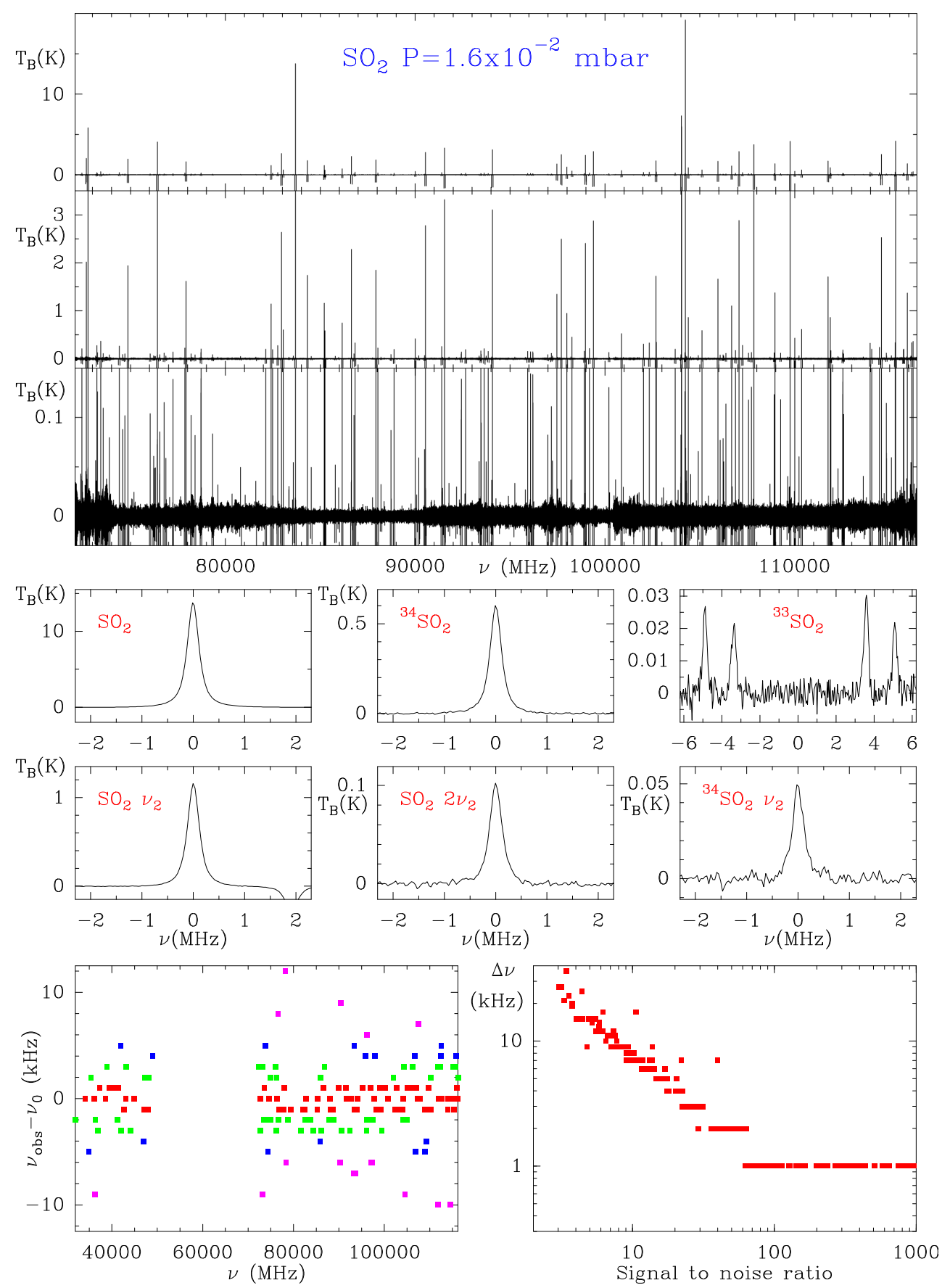

Fig. 13. Observed spectrum of $\mathrm{SO}_{2}$ in the $W$ band. The integration time per $W$ sub-band was $4.4 \mathrm{~h}$. Three upper panels: $W$ band with different intensity scales. Middle panels: $8_{1,7}-8_{0,8}$ transition of $\mathrm{SO}_{2},{ }^{34} \mathrm{SO}_{2},{ }^{33} \mathrm{SO}_{2}, \mathrm{SO}_{2} v_{2}$ and $2 v_{2}$, and ${ }^{34} \mathrm{SO}_{2} v_{2}$. The strongest hyperfine components of ${ }^{33} \mathrm{SO}_{2}$ are clearly detected. Data for all lines observed during this experiment are given in Table C.1. Bottom left panel: difference, $\Delta v(\mathrm{kHz})$, between the observed frequencies and those found in the CDMS (Müller et al. 2005), JPL (Pickett et al. 1998), and MADEX (Cernicharo 2012) catalogues. The colours correspond to different degrees of accuracy: $|\Delta v(\mathrm{kHz})| \leq 2$ (red; 75 lines), $2<|\Delta v(\mathrm{kHz})| \leq 4$ (green; 53 lines), $4<|\Delta v(\mathrm{kHz})| \leq 6$ (blue; 17 lines), and $6<|\Delta v(\mathrm{kHz})| \leq 12$ (purple; 16 lines). Bottom right panels: uncertainty (in $\mathrm{kHz}$ ) assigned by the Gaussian fitting algorithm of the CLASS/GILDAS program to all observed lines in the $W$ band as a function of the $\mathrm{S} / \mathrm{N}$ of the peak intensity. vibrational excited states of this species. Figure C.2 shows the same rotational transition for selected isotopologues and vibrationally excited states. The observing time in this experiment was $2.2 \mathrm{~h}$ per $W$ sub-band $(6.6 \mathrm{~h}$ in the $Q$ band). The selected observing mode was FS with a throw of $37 \mathrm{MHz}$ and a phase of $2 \mathrm{~s}$ for the frequency switch. As each rotational transition is observed in the ON and the OFF phases (shifted by $37 \mathrm{MHz}$ in this case) the data have to be folded to get the final spectrum and to improve the $\mathrm{S} / \mathrm{N}$. As a consequence of the folding, each emission feature is accompanied by two negative signals at $\pm 37 \mathrm{MHz}$ of the corresponding frequency.

From the observed intensities of the different isotopologues of OCS shown in Fig. C.2 we can derive the following isotopic abundance ratios: ${ }^{12} \mathrm{C} /{ }^{13} \mathrm{C}=92.8 \pm 0.4$ (93.5), ${ }^{32} \mathrm{~S} /{ }^{34} \mathrm{~S}=23.7 \pm 0.1(23.5),{ }^{32} \mathrm{~S} /{ }^{33} \mathrm{~S}=135.7 \pm 1.3$ (133.3), ${ }^{16} \mathrm{O} /{ }^{18} \mathrm{O}=481.3 \pm 20.0(487.8),{ }^{16} \mathrm{O} /{ }^{17} \mathrm{O}=2636 \pm 200.0(2630)$, and ${ }^{12} \mathrm{C} \times{ }^{32} \mathrm{~S} /{ }^{13} \mathrm{C} \times{ }^{34} \mathrm{~S}=2230 \pm 150.0$ (2199). The values in parentheses correspond to the natural abundances of the isotopic species (Juris et al. 2016). For lines of a given molecule observed with a $S / N>30$, the accuracy of the derived partial pressure is better than $3 \%$ (see also Appendix B). Hence, the quoted error bars on these isotopic abundance ratios vary between $0.5-1.0 \%$ for the most abundant isotopes $(S / N>100)$ to $10 \%$ for the rare isotopes $(S / N>10)$.

The vibrational modes $v_{2}, 2 v_{2}, 3 v_{2}, v_{1}, v_{3}$, and $v_{2}+v_{3}$ of OCS are detected with a high $\mathrm{S} / \mathrm{N}$ (see Fig. 12). The bending modes of $\mathrm{OC}^{34} \mathrm{~S}, \mathrm{OC}^{33} \mathrm{~S}$, and $\mathrm{O}^{13} \mathrm{CS}$ are also detected, together with the ground vibrational state rotational transitions of these isotopologues and those of ${ }^{18} \mathrm{OCS},{ }^{17} \mathrm{OCS}$, and $\mathrm{O}^{13} \mathrm{C}^{34} \mathrm{~S}$ (see Fig. C.2).

Finally, $\mathrm{SO}_{2}$ was also observed at a pressure of $1.6 \times$ $10^{-2}$ mbar (linewidths at half power intensity for most lines are 
J. Cernicharo et al.: Broad-band rotational spectroscopy for laboratory astrophysics
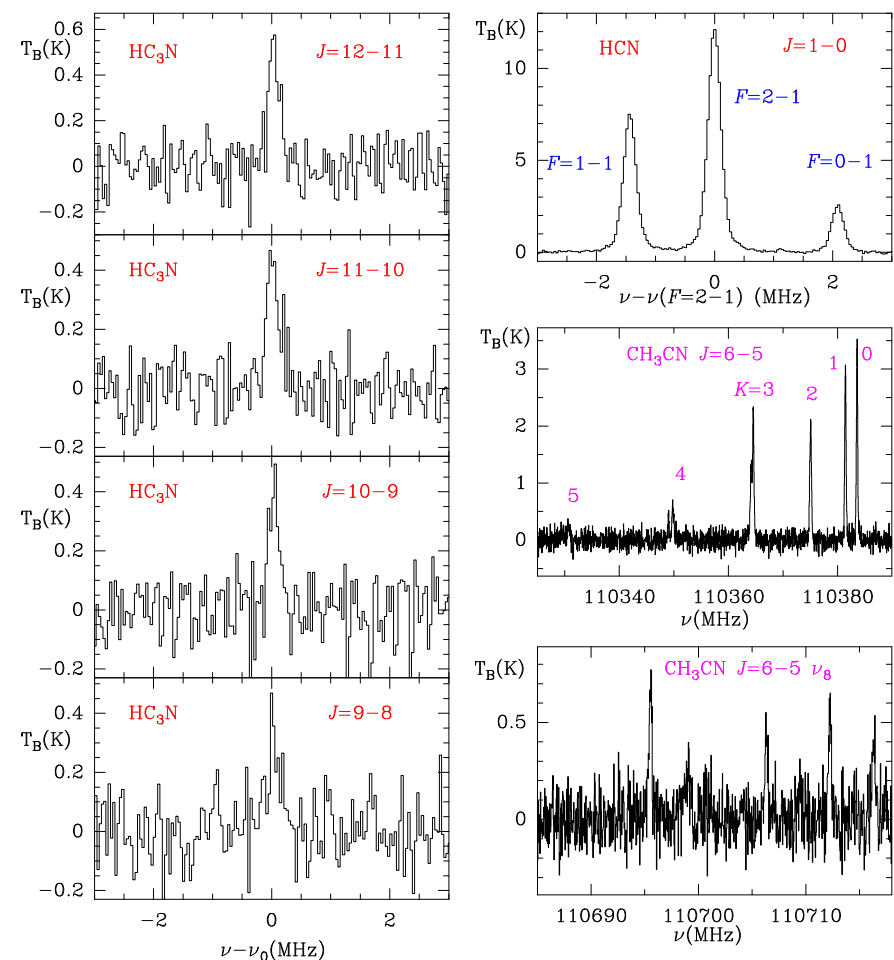

Fig. 14. Main spectral features found in the $W$ band during the short plasma experiment of $\mathrm{CH}_{3} \mathrm{CN}$. The most prominent features correspond to $\mathrm{HCN}$ (top right panel), followed by those of the precursor (middle right and bottom right panels). The four rotational lines of $\mathrm{HC}_{3} \mathrm{~N}$ falling in the $W$ band are detected (left panels). The expected intensity of the $J=2 \rightarrow 1$ transition in $Q$ band was too low to be detected in the short observing time of the experiment $(80 \mathrm{~s})$. The observing procedure was $\mathrm{ON} / \mathrm{OFF}$ using the ICL as OFF.

$\sim 300-350 \mathrm{kHz}$; see Table C.1). Figure 13 shows the whole $W$ band observed during this experiment. The lines corresponding to the $8_{1,7}-8_{0,8}$ transition of $\mathrm{SO}_{2}$, its isotopologues, and the vibrationally excited states are also shown. All line parameters for the features observed in this experiment are reported in Table C. 1 and have been used to check the frequency calibration of the system and the uncertainty in deriving frequencies as a function of the data $\mathrm{S} / \mathrm{N}$. The bottom left panel of Fig. 13 shows the difference between the observed frequencies and those reported in the CDMS (Müller et al. 2005), JPL (Pickett et al. 1998), or MADEX catalogues (Cernicharo 2012). Since the observed isotopologues of $\mathrm{SO}_{2}$ have been measured previously up to $1-2 \mathrm{THz}$ (i.e. up to high- $J$ and high- $K$ transitions) with high accuracy by different authors, the frequencies for their rotational transitions in these catalogues are accurate to $1 \mathrm{kHz}$, or even better, in the $Q$ and $W$ bands. From the whole set of observed lines, we selected 161 features corresponding to $\mathrm{SO}_{2},{ }^{34} \mathrm{SO}_{2}$ and ${ }^{33} \mathrm{SO}_{2}$, for which the $\mathrm{S} / \mathrm{N}$ is larger than 20 . We can see that $47 \%$ of the frequency differences are below $2 \mathrm{kHz}$ ( 75 features), and this percentage becomes as high as $80 \%$ when differences of up to $4 \mathrm{kHz}$ are considered. Hence, we can conclude that the frequency calibration of the system is excellent. The complete data set for $\mathrm{SO}_{2}$ in the $Q$ and $W$ bands are shown in Fig. C.3.

The bottom right panel of Fig. 13 shows the uncertainty of the observed frequencies, obtained by fitting a Gaussian profile to the lines, as a function of the $\mathrm{S} / \mathrm{N}$ of the line intensities. For $\mathrm{S} / \mathrm{N}$ higher than 10 the uncertainty is below $7 \mathrm{kHz}$, being $\sim 2 \mathrm{kHz}$ for $S / N \sim 20$, and $\sim 1 \mathrm{kHz}$ for larger $\mathrm{S} / \mathrm{N}$.

\section{Cold plasmas}

Cold plasmas provide very reactive media at room temperature. Fast electrons, with energies in the tens of $\mathrm{eV}$, break the molecules and lead to the production of reactive ions and radicals that can initiate a complex chemistry both in the gas phase and at the vessel walls (Tanarro et al. 2011). They have been used at the IEM group for the study of species of astrophysical interest (e.g. Carrasco et al. 2012; Doménech et al. 2013, 2016; Cueto et al. 2014). In the following, we comment on the effects of coupling a plasma on some gases and gas mixtures in our chamber. In these experiments the plasma incident power provided by the RF source was between 20 and $35 \mathrm{~W}$. Approximately one-half of the power was reflected.

Mass spectra were also used to observe the change in chemical composition upon plasma ignition. Additional information about the plasma products was derived from optical emission measurements.

\section{1. $\mathrm{CH}_{3} \mathrm{CN}$}

A short experiment ( $80 \mathrm{~s}$ integration time) was first performed (in $\mathrm{ON} / \mathrm{OFF}$ mode) with $\mathrm{CH}_{3} \mathrm{CN}$, immersed in a bath at $30^{\circ} \mathrm{C}$ in order to avoid fluctuations of the vapour pressure. The residual pressure in the chamber before injecting $\mathrm{CH}_{3} \mathrm{CN}$ was $10^{-4}$ mbar, mostly due to residual air. The pressure in the chamber was $3 \times$ $10^{-3}$ mbar at the beginning of the data acquisition.

Figure 14 displays the main rotational lines from the $\mathrm{CH}_{3} \mathrm{CN}$ plasma in the $W$ band. The main products observed were $\mathrm{HCN}$ and $\mathrm{HC}_{3} \mathrm{~N}$. The optical emission spectrum (very similar to the one shown in Fig. 18) indicated the presence of $\mathrm{CN}$ in the gas phase, but it was not detected in rotational spectroscopy in this short observing time. The expected intensities in the $Q$ band for the products and the precursor were too weak to be detected in $80 \mathrm{~s}$.

From the observed line intensities we derive the following partial pressures in the chamber: $P(\mathrm{HCN})=0.39 \times 10^{-3} \mathrm{mbar}$ and $P\left(\mathrm{HC}_{3} \mathrm{~N}\right)=6 \times 10^{-6}$ mbar (both corrected for their corresponding vibrational partition function at $300 \mathrm{~K}$ ). We note that this low partial pressure of $\mathrm{HC}_{3} \mathrm{~N}$ corresponds to $n\left(\mathrm{HC}_{3} \mathrm{~N}\right)=1.5 \times$ $10^{11} \mathrm{~cm}^{-3}$ and $N\left(\mathrm{HC}_{3} \mathrm{~N}\right)=1.4 \times 10^{13} \mathrm{~cm}^{-2}$. The partial pressure of the precursor can be derived from the $J=6 \rightarrow 5$ transition (see Fig. 14) to be $P\left(\mathrm{CH}_{3} \mathrm{CN}\right)=3 \times 10^{-4} \mathrm{mbar}$ (taking into account the $A$ and $E$ species and the contribution of the vibrational modes to the partition function). That means that only one-tenth of the precursor remains unscathed in the cold plasma. With the discharge $\mathrm{ON}$, the mass spectra indicated that the concentration of $\mathrm{CH}_{3} \mathrm{CN}$ decreased by $85 \%$ (consistent with the observed intensities in the rotational spectrum) and that a large amount of hydrogen was formed in the chamber. The production of $\mathrm{HCN}$ was also observed by the growth of the peak at mass 27. There is also an observable decrease in the peak of background $\mathrm{O}_{2}$ at mass 32 and a growth in the signals of $\mathrm{CO}$ and $\mathrm{CO}_{2}$. Although not detectable by its rotational spectrum, $\mathrm{N}_{2}$ was clearly visible in the mass spectrum and in the optical spectrum (see Figs. 17 and 18 showing very similar results for a $\mathrm{N}_{2}+\mathrm{CH}_{4}$ plasma). From the difference of pressures between that of the precursor before the plasma was switched on and those derived for $\mathrm{HCN}, \mathrm{HC}_{3} \mathrm{~N}$, and the remaining $\mathrm{CH}_{3} \mathrm{CN}$, we conclude that the species $\mathrm{H}_{2}, \mathrm{~N}_{2}, \mathrm{CH}_{4}$, and perhaps $\mathrm{C}_{2} \mathrm{H}_{2}$, contribute with a pressure of $1.3 \times 10^{-3}$ mbar to the total pressure in the cell. An increase of $\sim 100 \mathrm{~K}$ in the vibrational temperature of $\mathrm{CH}_{3} \mathrm{CN}$ is derived from the analysis of the intensity ratio of the rotational lines from the ground state and those from its $v_{8}$ vibrational 


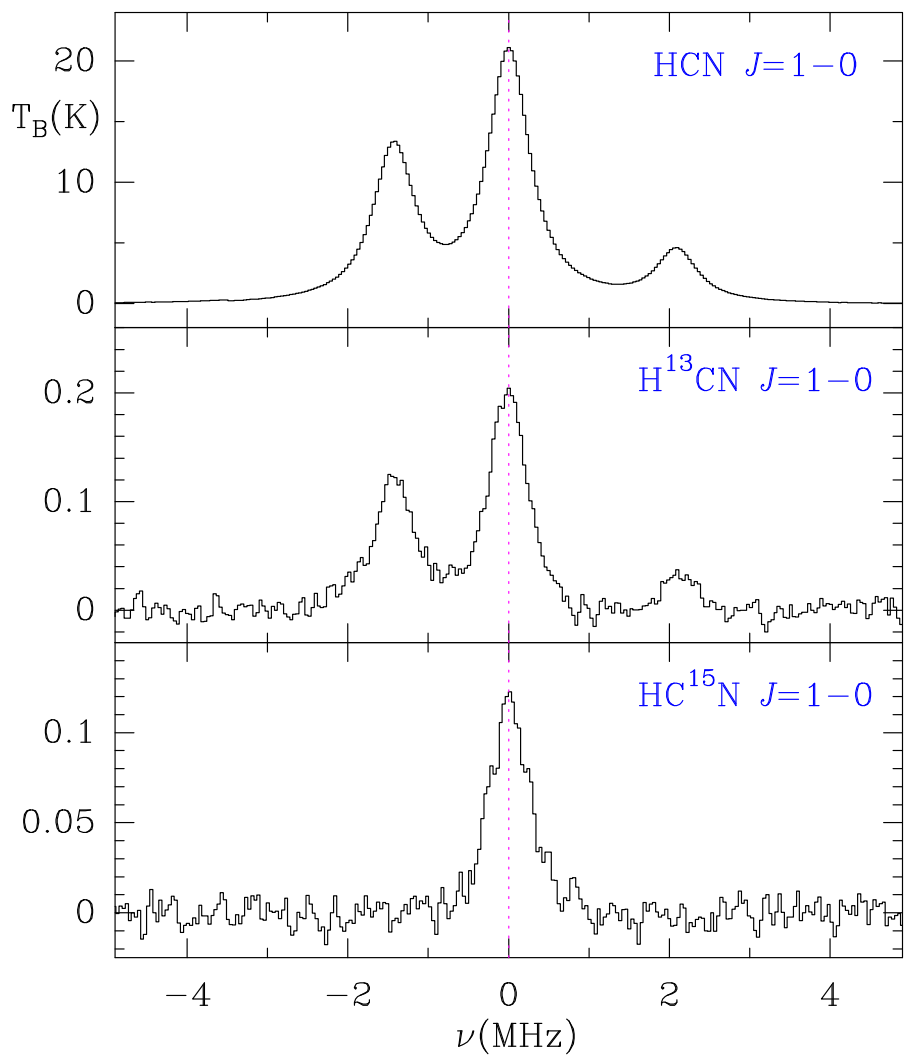

Fig. 15. The $J=1 \rightarrow 0$ line of the three isotopologues of $\mathrm{HCN}$ observed in the plasma of $\mathrm{CH}_{4}+\mathrm{N}_{2}+\mathrm{CH}_{3} \mathrm{CN}$ (26 min of observing time per $W$ sub-band). This frequency domain of the spectrum is observed in the WL and WM sub-bands. Hence, the total observing time is $52 \mathrm{~min}$. The observing procedure was FS with a throw of $37 \mathrm{MHz}$. The line width at half intensity is $0.62 \mathrm{MHz}$ for a total pressure of $28 \times 10^{-3} \mathrm{mbar}$. Frequencies are relative to the strongest hyperfine component.

mode. The formation of $\mathrm{HC}_{3} \mathrm{~N}$ can proceed via the formation of $\mathrm{C}_{2} \mathrm{H}_{2}$ in the chamber $\left(\mathrm{C}_{2} \mathrm{H}_{2}+\mathrm{CN}\right.$ and $\left.\mathrm{CCH}+\mathrm{HCN}\right)$, or from the reaction of the radicals $\mathrm{CCN}, \mathrm{HCCN}$, and $\mathrm{H}_{2} \mathrm{CCN}$ (all of them fragmentation products of $\mathrm{CH}_{3} \mathrm{CN}$ ) with $\mathrm{CH}_{2}$ and/or $\mathrm{CH}_{3}$. Reactions on the walls could also produce cyanoacetylene $\left(\mathrm{HC}_{3} \mathrm{~N}\right)$.

\section{2. $\mathrm{CH}_{3} \mathrm{OH}$}

After the $\mathrm{CH}_{3} \mathrm{CN}$ experiment, a $100 \mathrm{~s}$ integration was performed in ON/OFF mode with the chamber filled with $\mathrm{CH}_{3} \mathrm{OH}$ at a pressure of $20 \times 10^{-3}$ mbar. The cell walls were not cleaned after the previous short experiment with the $\mathrm{CH}_{3} \mathrm{CN}$ plasma. The strongest feature we found in the spectra was HCN, followed by $\mathrm{CO}$, most probably as a consequence of the adsorbed molecules and adhered dust from the previous experiment. Weak lines of $\mathrm{CH}_{3} \mathrm{OH}$ were also detected indicating that about $10 \%$ of the initial methanol molecules were able to survive in the plasma. No lines of $\mathrm{CH}_{3} \mathrm{CN}$ were detected indicating that most of the molecules in the walls are polymers of HCN. From the observed intensities, the following partial pressures were found: $P(\mathrm{HCN})=8.5 \times 10^{-5} \mathrm{mbar}, P(\mathrm{CO})=8 \times 10^{-3} \mathrm{mbar}$, and $P\left(\mathrm{CH}_{3} \mathrm{OH}\right)=5 \times 10^{-3}$ mbar. With respect to the initial pressure of the precursor, $7 \times 10^{-3}$ mbar are still missing, which correspond to $\mathrm{H}_{2}, \mathrm{H}_{2} \mathrm{O}$, and $\mathrm{CO}_{2}$ as fragmentation products of $\mathrm{CH}_{3} \mathrm{OH}$ in the plasma, as seen by mass spectrometry. The column density of $\mathrm{HCN}$ in the chamber was $2.0 \times 10^{14} \mathrm{~cm}^{-2}$.

In order to check our ability to introduce two liquids in a controlled way in the chamber, we performed a short observation
(100 s) of a mixture of $\mathrm{CH}_{3} \mathrm{CN}$ and $\mathrm{CH}_{3} \mathrm{OH}$ with a total pressure of $13 \times 10^{-3}$ mbar with no $\mathrm{RF}$ coupling. The lines of $\mathrm{CH}_{3} \mathrm{CN}$ in the ground state and in the $v_{8}$ and $2 v_{8}$ states were very strong and easily detected in this short observing time. Although this experiment was performed just after the $\mathrm{CH}_{3} \mathrm{CN}$ and $\mathrm{CH}_{3} \mathrm{OH}$ plasmas, no $\mathrm{HCN}$ was detected in this short integration time, as expected, since the plasma was switched off and therefore there was no mechanism to remove molecules from the wall.

We used the same gas composition in the chamber, $\mathrm{CH}_{3} \mathrm{CN}$ and $\mathrm{CH}_{3} \mathrm{OH}$, to initiate a plasma with a total initial pressure of $30 \times 10^{-3}$ mbar. The main polar products observed in the plasma gas, in addition to the precursors, are $\mathrm{HCN}, \mathrm{HC}_{3} \mathrm{~N}$, and $\mathrm{CO}$. With respect to the pure $\mathrm{CH}_{3} \mathrm{CN}$ plasma, the only new product is $\mathrm{CO}$. In comparison with the plasma of pure $\mathrm{CH}_{3} \mathrm{OH}$ the only new species found is $\mathrm{HC}_{3} \mathrm{~N}$ (we note, however, that $\mathrm{HCN}$ did come from the walls in this case). The integration time for this experiment was also $100 \mathrm{~s}$.

\section{3. $\mathrm{CH}_{4} / \mathrm{N}_{2}$}

The next plasma studied was induced in a flowing mixture of $\mathrm{CH}_{4}$ and $\mathrm{N}_{2}$ with a contribution of methane and molecular nitrogen of $13.4 \times 10^{-3}$ and $19 \times 10^{-3}$ mbar, respectively. Since both molecules are non-polar, no rotational lines from them are observed in the $Q$ or $W$ band.

In this case, the integration time was 40 min per $W$ sub-band and the observing mode was FS with a throw of $37 \mathrm{MHz}$. The rotational spectrum shows the presence of $\mathrm{HCN}, \mathrm{HC}_{3} \mathrm{~N}$, and $\mathrm{CH}_{3} \mathrm{CN}$ as the main products of the reactions between the fragments of the precursors in the plasma (see Figs. 15, 16, and C.4). From the observed intensities, we derive the partial pressures of $10^{-5}$ mbar, $4 \times 10^{-4}$ mbar, and $1.5 \times 10^{-5}$ mbar for $\mathrm{CH}_{3} \mathrm{CN}, \mathrm{HCN}$, and $\mathrm{HC}_{3} \mathrm{~N}$, respectively. The corresponding column densities are $2.2 \times 10^{13}, 9.0 \times 10^{14}$, and $3.3 \times 10^{13} \mathrm{~cm}^{-2}$. It is obvious that the partial pressures are far from the total pressure in the chamber $\left(32.4 \times 10^{-3}\right.$ mbar). Most of the missing gases have to be the precursors $\mathrm{CH}_{4}$ and $\mathrm{N}_{2}$, with other non-polar products (e.g. $\mathrm{H}_{2}$, $\mathrm{C}_{2} \mathrm{H}_{2}, \mathrm{C}_{2} \mathrm{H}_{6}$ ).

We monitored the variations in these species through the mass spectrometer (see Fig. 17). In addition to the fuel gases $\mathrm{N}_{2}$ and $\mathrm{CH}_{4}$, the mass spectrum with the plasma OFF showed the presence of small amounts of $\mathrm{O}_{2}, \mathrm{H}_{2} \mathrm{O}, \mathrm{CO}_{2}$, and $\mathrm{Ar}$ from the background gas. With the plasma $\mathrm{ON}$, the mass peaks at 15 and $16\left(\mathrm{CH}_{4}\right)$ decrease by $50 \%$ and that of mass $28\left(\mathrm{~N}_{2}\right)$ by about $5 \%$. There is a large increase in the peaks of $\mathrm{H}_{2}$ (masses 1 and 2) and also an appreciable growth in the peaks at masses 26 and 27 (HCN), and masses 29 and 30, which correspond most probably to fragments of small hydrocarbons $\left(\mathrm{C}_{\mathrm{x}} \mathrm{H}_{\mathrm{y}}\right)$. The formation of $\mathrm{CH}_{3} \mathrm{CN}$ and $\mathrm{HC}_{3} \mathrm{~N}$ is observed by their rotational lines and is further corroborated by the appearance of peaks at masses 41 and 51 , respectively. The optical spectrum observed for these plasma types is shown in Fig. 18. It is dominated by $\mathrm{N}_{2}$ bands, although signals coming from $\mathrm{N}_{2}^{+}$and $\mathrm{CN}$ were also detected.

$\mathrm{N}_{2}$ is a very stable species and it is only marginally fragmented (less than 5\%) with the power used in the RF generator for this plasma $(20 \mathrm{~W})$ as seen in the mass spectrum of the plasma (see Fig. 17). However, about one-half of $\mathrm{CH}_{4}$ is fragmented. We have used several plasmas with different $\mathrm{CH}_{4} / \mathrm{N}_{2}$ abundance ratios to test the effect of the relative abundances in that of the products $\mathrm{HCN}$ and $\mathrm{HC}_{3} \mathrm{~N}$. It is found that decreasing the methane abundance has an important impact on the abundance of $\mathrm{HC}_{3} \mathrm{~N}$. These results, which can be of help in interpreting the chemistry of the upper layers of Titan's atmosphere, will be published elsewhere. It seems that the limiting factor in 

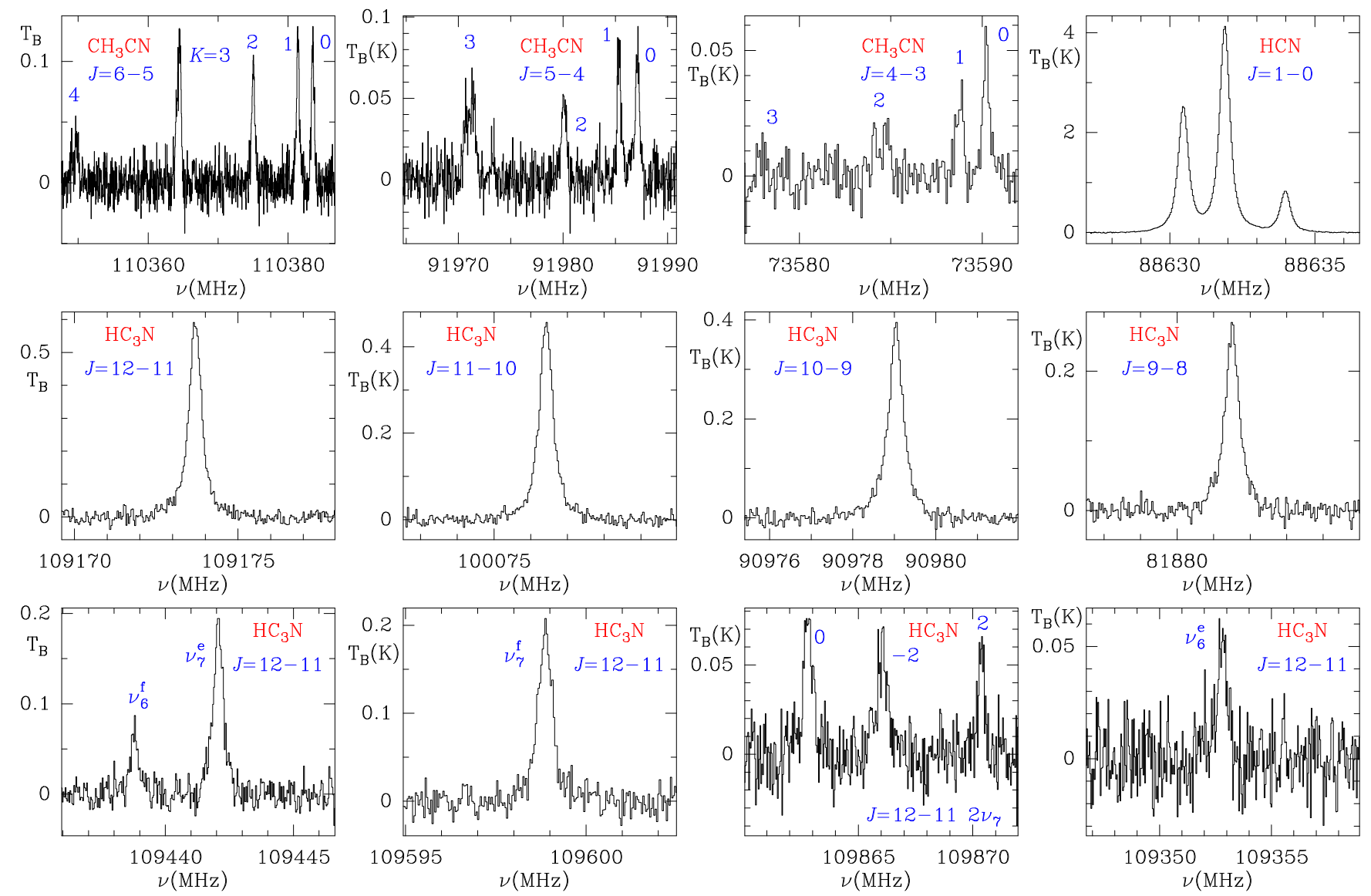

Fig. 16. Selected lines of $\mathrm{CH}_{3} \mathrm{CN}, \mathrm{HCN}$, and $\mathrm{HC}_{3} \mathrm{~N}$ observed in the plasma of a flowing mixture of $\mathrm{CH}_{4}$ and $\mathrm{N}_{2}$ with a total pressure of $32.4 \times$ $10^{-3}$ mbar. The integration time was $40 \mathrm{~min}$ in each $W$ sub-band. The observing procedure was frequency switching with a throw of $37 \mathrm{MHz}$. The four rotational transitions of the ground state of $\mathrm{HC}_{3} \mathrm{~N}$ within the $W$ band are shown in the middle panels. The different components of the $J=12 \rightarrow 11$ rotational transition of $\mathrm{HC}_{3} \mathrm{~N}$ in its vibrationally excited states $v_{6}, v_{7}$, and $2 v_{7}$ are shown in the bottom panels.

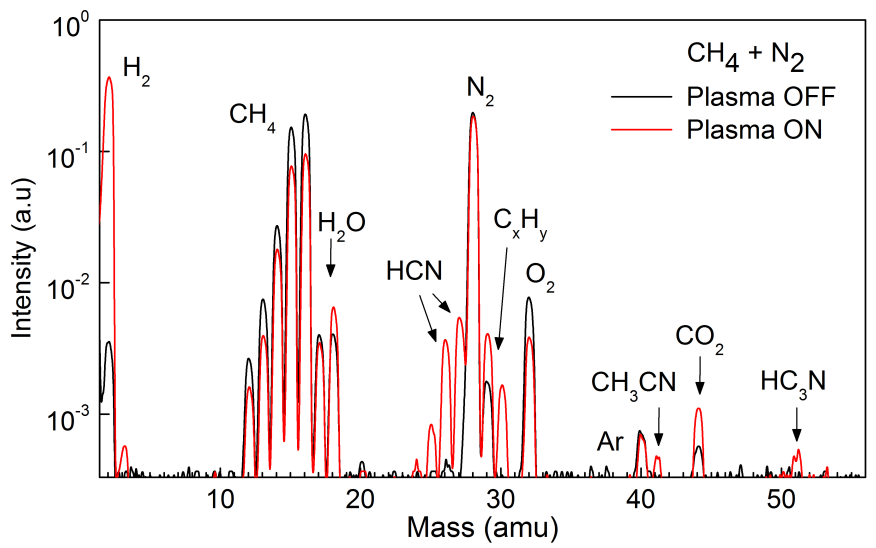

Fig. 17. Mass spectrum taken when a plasma was produced in a flowing mixture of $\mathrm{CH}_{4}(1 \mathrm{sccm})$ and $\mathrm{N}_{2}(1 \mathrm{sccm})$. The optical spectrum observed during the same plasma is shown in Fig. 18, and results from rotational spectroscopy during this plasma experiment are shown in Fig. 16.

the chemistry of these plasmas is the residence time in the gas phase of atoms $(\mathrm{C}, \mathrm{N}, \mathrm{H})$ and radicals $\left(\mathrm{CCH}, \mathrm{CN}, \mathrm{HCCN}, \mathrm{CH}_{2}\right.$, $\mathrm{CH}_{3}, \mathrm{H}_{2} \mathrm{CCN}$ ) before reaching the cell walls. The power of the $\mathrm{RF}$ generator also plays a role in the fragmentation of the precursors. We have found that increasing the RF power from 20 to $35 \mathrm{~W}$ increases the production of $\mathrm{HCN}$ by a factor of 2 .

\section{4. $\mathrm{CH}_{4} / \mathrm{N}_{2} / \mathrm{CH}_{3} \mathrm{CN}$}

The last example of this section is that of a plasma induced in a flowing mixture of $\mathrm{CH}_{4}, \mathrm{~N}_{2}$, and $\mathrm{CH}_{3} \mathrm{CN}$, with an $\mathrm{RF}$ power of $35 \mathrm{~W}$ and a total pressure in the chamber of $28 \times 10^{-3}$ mbar. The observing time was $26 \mathrm{~min}$ and the observing mode was FSW with a throw of $37 \mathrm{MHz}$. The partial pressures of the three gases were identical. This plasma produces a forest of lines (see Fig. C.5), and shows very strong lines of HCN (see Fig. 15) and $\mathrm{HC}_{3} \mathrm{~N}$ (see Fig. C.6). In addition, species such as $\mathrm{CH}_{2} \mathrm{CHCN}$ and $\mathrm{CH}_{3} \mathrm{CH}_{2} \mathrm{CN}$ (including some of their low-energy bending and torsion modes) are detected (see Fig. C.6). The vibrationally excited states of $\mathrm{HC}_{3} \mathrm{~N} v_{4}, v_{5}, v_{6}, v_{7}, 2 v_{7}, 3 v_{7}, 4 v_{7}, v_{5}+v_{7}, v_{6}+v_{7}$, and $2 v_{6}+v_{7}$ are detected with very good S/N. As in other plasmas studied with the GACELA set-up the vibrational temperature seems to be $100-150 \mathrm{~K}$ above the ambient temperature (see also Tanarro et al. 2018).

The intensity of $\mathrm{HCN}$ is so high that the isotopologues $\mathrm{H}^{13} \mathrm{CN}$ and $\mathrm{HC}^{15} \mathrm{~N}$ are nicely observed (see Fig. 15). Even the three single substituted ${ }^{13} \mathrm{C}$ species of $\mathrm{HC}_{3} \mathrm{~N}$ are detected. The spectra shown in Figs. 15, C.5, and C.6 have a high sensitivity; the HCN data from different plasmas can be used to derive the abundance of the isotopologues of $\mathrm{HCN}$. We obtain $\mathrm{HCN} / \mathrm{H}^{13} \mathrm{CN}=91.0 \pm 1.8$ and $\mathrm{HCN} / \mathrm{HC}^{15} \mathrm{~N}=279 \pm 8$, i.e. the natural abundance of ${ }^{13} \mathrm{C}$ and ${ }^{15} \mathrm{~N}$ relative to ${ }^{12} \mathrm{C}$ and ${ }^{14} \mathrm{~N}$, respectively (Juris et al. 2016). These results, together with those relative to OCS and analysed in Sect. 3.2, show that the high accuracy in measuring molecular abundances that can 

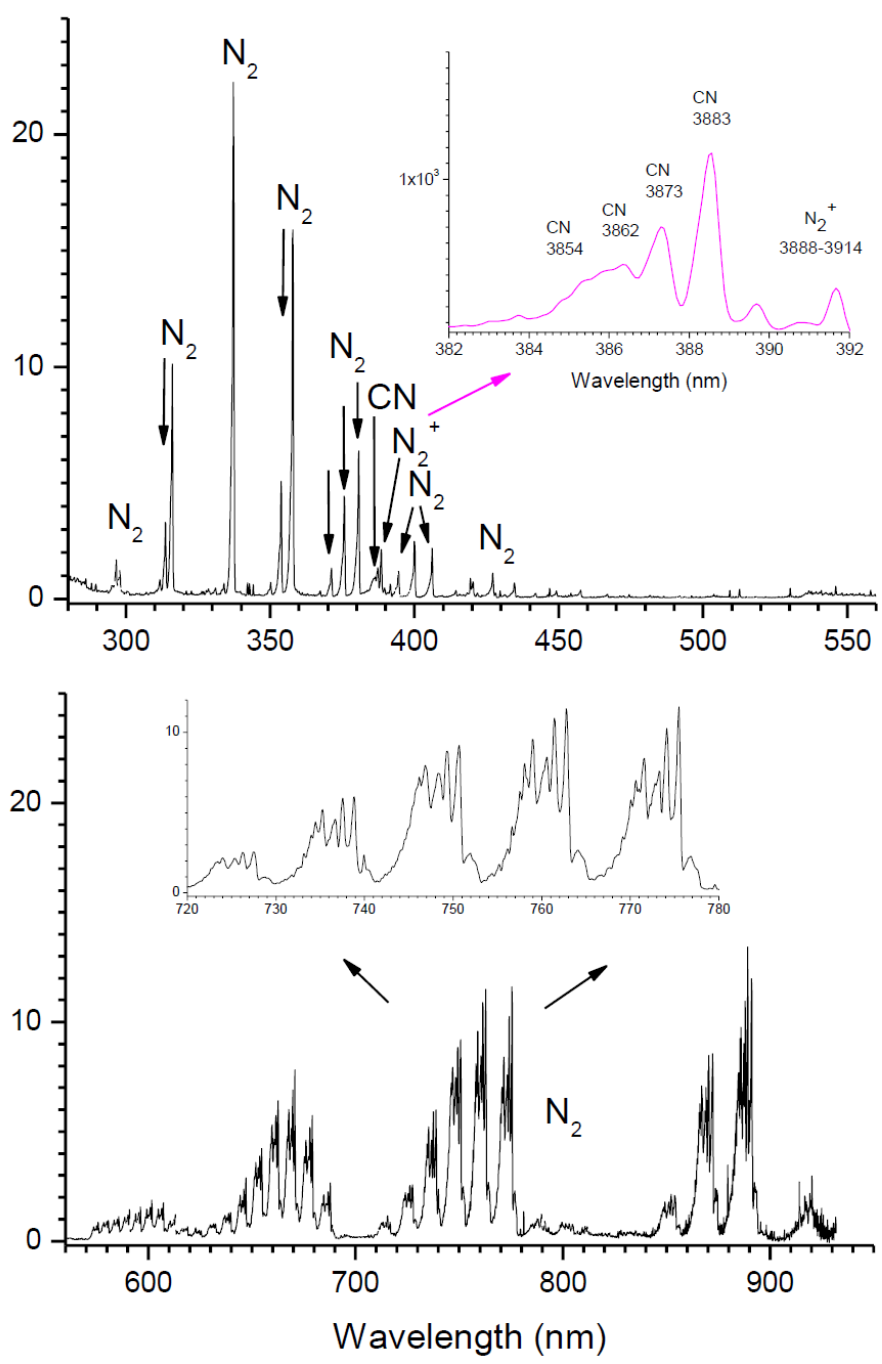

Fig. 18. Optical spectrum between 280 and $560 \mathrm{~nm}$ (top) and between 560 and $950 \mathrm{~nm}$ (bottom) of a plasma produced in a flowing mixture of $\mathrm{CH}_{4}(1 \mathrm{sccm})$ and $\mathrm{N}_{2}(1 \mathrm{sccm})$. The inserts show the $\mathrm{CN}, \mathrm{N}_{2}^{+}$, and $\mathrm{N}_{2}$ bands. The corresponding mass spectrum is shown in Fig. 17, and the results from the rotational spectroscopy during this plasma experiment are shown in Fig. 16.

be reached with GACELA. We have also detected $\mathrm{CO}$, which is a low-abundance residual gas in the chamber. Nevertheless, it can be also formed from $\mathrm{CH}_{4}$ and residual $\mathrm{O}_{2}$ and/or $\mathrm{H}_{2} \mathrm{O}$ in the chamber. The FWHM of most features is $0.65-$ $0.70 \mathrm{MHz}$, which indicates an average collisional broadening of $12.5 \mathrm{MHz} \mathrm{mbar}^{-1}$ (HWHM). We have estimated the following partial pressures: $P(\mathrm{CO})=0.4 \times 10^{-3}$ mbar, $P(\mathrm{HCN})=2.3 \times$ $10^{-3}$ mbar, $P\left(\mathrm{HC}_{3} \mathrm{~N}\right)=0.6 \times 10^{-3}$ mbar (corrected for the contribution of the vibrational modes to the partition function), $P\left(\mathrm{CH}_{3} \mathrm{CN}\right)=7.0 \times 10^{-3}$ mbar, $P\left(\mathrm{CH}_{2} \mathrm{CHCN}\right)=0.02 \times 10^{-3} \mathrm{mbar}$, and $P\left(\mathrm{CH}_{3} \mathrm{CH}_{2} \mathrm{CN}\right)=0.10 \times 10^{-3} \mathrm{mbar}$. The missing pressure of $18 \times 10^{-3}$ mbar is probably due to $\mathrm{H}_{2}, \mathrm{~N}_{2}, \mathrm{CH}_{4}, \mathrm{C}_{2} \mathrm{H}_{2}$, and $\mathrm{C}_{2} \mathrm{H}_{6}$; a large growth in the concentration of $\mathrm{H}_{2}$ and a strong decrease in the mass peaks corresponding to $\mathrm{CH}_{3} \mathrm{CN}(m=38-41 \mathrm{u})$ are observed in the mass spectra of the plasma. A significant increase in the signal is also observed for the mass peaks 2630 , corresponding probably to small hydrocarbons and notably to $\mathrm{HCN}(m=27 \mathrm{u})$. Much smaller signals appearing in the 5054 mass range can be associated with $\mathrm{CH}_{3} \mathrm{CH}_{2} \mathrm{CN}, \mathrm{CH}_{2} \mathrm{CHCN}$, and $\mathrm{HC}_{3} \mathrm{~N}$, which are seen in rotational spectroscopy.
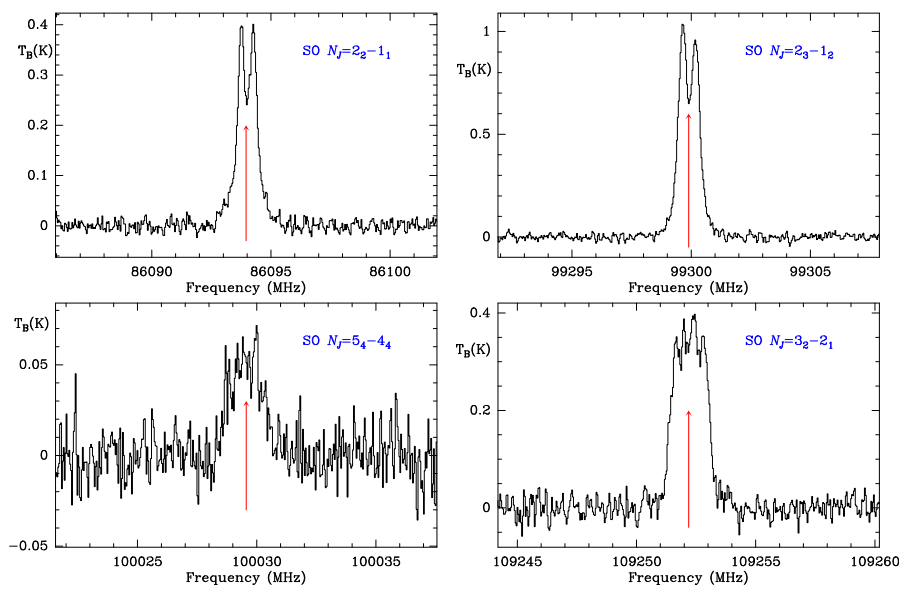

Fig. 19. Rotational lines of the radical SO observed while cleaning the walls with a plasma of $\mathrm{O}_{2}$ just after a plasma of OCS was produced. The lines are Zeeman split due to the Earth's magnetic field.

\subsection{Other plasmas and cleaning of the walls}

During the commissioning of GACELA, several additional plasmas were studied. Some of them quickly produced a large deposit of material on the walls. The plasma of $\mathrm{CH}_{4} / \mathrm{N}_{2}$ and $\mathrm{CH}_{4} / \mathrm{N}_{2} / \mathrm{CH}_{3} \mathrm{CN}$ produced a thin film of brown material (tholins; see Sagan \& Khare 1979) on the walls and on the copper coil of the RF generator. As commented in Sect. 2.1, to avoid the direct contamination of the steel of the chamber, it was covered with a $2 \mathrm{~mm}$ thick lining of Teflon ${ }^{\mathrm{TM}}$ (see Fig. A.2). The RF coil was also covered with $50 \mu \mathrm{m}$ of Teflon ${ }^{\mathrm{TM}}$. The deposit of tholins and other materials was directly monitored by means of a webcam installed in one of the viewports. Some plasmas such as those of OCS or $\left(\mathrm{CH}_{3}\right)_{3} \mathrm{SiOSi}\left(\mathrm{CH}_{3}\right)_{3}$ (Hexamethyldisiloxane; HMDSO) produced a thin deposit of sulfur and silicon compounds on the Teflon $^{\mathrm{TM}}$.

Cleaning the walls with a plasma of $\mathrm{O}_{2}$ produces different species in the gas phase. In the case of cleaning the material produced by a plasma of OCS, the most abundant polar species in the gas phase are $\mathrm{SO}_{2}, \mathrm{~S}_{2} \mathrm{O}$, OCS, CS, CO, and the radical SO. The observed lines of SO during these cleaning procedures are shown in Fig. 19. The lines show Zeeman splitting due to the Earth's magnetic field and the paramagnetic character of the SO molecule $\left({ }^{3} \Sigma^{-}\right)$(see, e.g., Pardo et al. 1995). The whole spectrum observed in the $W$ band during the $\mathrm{O}_{2}$ plasma cleaning is mainly dominated by the rotational lines of $\mathrm{SO}_{2}$ and is shown in Fig. C.7 (to be compared with the spectrum of $\mathrm{SO}_{2}$ shown in Figs. 13 and C.3). When the chamber was subsequently submitted to a $\mathrm{N}_{2}$ plasma, the NS radical was also found.

The SO radical is produced in an efficient and continuous way when the $\mathrm{O}_{2}$ plasma is active, probably due to the formation of $\mathrm{SO}_{2}$ in the walls, which is then easily fragmented into SO in the plasma. The radical SO reacts very slowly with $\mathrm{O}_{2}$. The reaction has a barrier of $\sim 2300 \mathrm{~K}$ (DeMore et al. 1997; Atkinson et al. 1997), and a rate at $300 \mathrm{~K}$ of $\simeq 8 \times$ $10^{-17}$ molec $\mathrm{cm}^{3} \mathrm{~s}^{-1}$. The three-body association $\mathrm{SO}+\mathrm{O}+\mathrm{M}$ is very slow at the pressures of our experiments. It seems that the reaction of SO with other gas compounds in the chamber is also rather slow (SSO, OCS, CS, and the fragments of the residual gases $\mathrm{H}_{2} \mathrm{O}, \mathrm{N}_{2}$, and $\mathrm{CO}_{2}$ ). To the best of our knowledge, there are no data on the reactivity of NS with $\mathrm{O}_{2}$ and O. From our observations, it seems that these reactions also have low rates.

When the cleaning was performed after the plasma of HMDSO, we found the same compounds as those found when 
cleaning was done after a plasma of $\mathrm{CH}_{4} / \mathrm{N}_{2}$. The fragmentation of HMDSO produces the radical $\mathrm{CH}_{3}$ (Basner et al. 1998; Alexander et al. 1997), while the fragments containing silicon stick immediately to the walls and the RF coil, and also form dust particles (Despax et al. 2016). The cleaning process did not produce any molecule containing silicon, but those typical of $\mathrm{CH}_{4}$ plasmas, i.e. $\mathrm{HCN}, \mathrm{HC}_{3} \mathrm{~N}$, and $\mathrm{CH}_{3} \mathrm{CN}$.

\section{N-ethylformamide: $\mathrm{CH}_{3} \mathrm{CH}_{2} \mathrm{NHCHO}$}

\subsection{Rotational spectroscopy}

Only a few molecules in space contain the peptide group $\mathrm{X}-\mathrm{CONH}-\mathrm{Y}$. Among them, formamide $\left(\mathrm{NH}_{2} \mathrm{CHO} ; \mathrm{X}=\mathrm{H}, \mathrm{Y}=\mathrm{H}\right)$ has attracted special attention in recent years due to its prebiotic character (see e.g. Ceccarelli et al. 2017; Ligterink et al. 2018; Botta et al. 2018). The next simplest species containing the peptide group is methylformamide, $\mathrm{CH}_{3} \mathrm{NHCHO}$, which has been characterized in the laboratory and tentatively detected in space by Belloche et al. (2017). It has also been searched for, but with negative results, towards IRAS 16923B by Ligterink et al. (2018). This species could be formed in ices (Ligterink et al. 2018) through the hydrogenation of methyl isocyanate, $\mathrm{CH}_{3} \mathrm{NCO}$, a molecule found to be very abundant in warm astrophysical environments (Cernicharo et al. 2016).

$\mathrm{CH}_{3} \mathrm{CH}_{2} \mathrm{NHCHO}$ (N-ethylformamide) is the next potential member of this series of peptide molecules to be present in space. Its rotational spectrum has been studied in the laboratory up to $13.4 \mathrm{GHz}$ by Ohba et al. (2005), and up to $20.5 \mathrm{GHz}$ by Vaquero-Vara et al. (2017). However, it is difficult to extrapolate these measurements up to the millimetre domain to search for it in the 3,2, and $1 \mathrm{~mm}$ line surveys of astrophysical sources showing a rich molecular content.

In order to search for this interesting species in space we have studied the rotational spectrum of NEFA in the $Q$ and $W$ bands using the GACELA set-up. The sample of NEFA (purity >98\%) was purchased from TCI Chemicals and was used without further purification. The sample was placed in a Pyrex ${ }^{\mathrm{TM}}$ vacuum Schlenk tube, and was degassed using the common freeze-pumpthaw cycling method through a $3 \mathrm{~m}^{3} \mathrm{~h}^{-1}$ rotary pump (Pfeiffer Duo $3 \mathrm{M}$ ) that allows pressure control in the sample injection line. Inlet connections from the Schlenk tube to the chamber were made using a Teflon ${ }^{\mathrm{TM}}$ tube about $20 \mathrm{~cm}$ in length. In order to avoid liquid condensation during the injection, the temperature of the sample container and the injection line was kept at $40^{\circ} \mathrm{C}$ using a dry heating tape and a proportional-integralderivative (PID) temperature controller (Omega CN740). Prior to the sample introduction, the pressure inside the vacuum chamber was $2.0 \times 10^{-4}$ mbar; during the experiment, carried out in flow mode, the pressure was kept at $3.0 \times 10^{-3} \mathrm{mbar}$ because higher pressures produce undesirable line broadenings. We found that the molecule has a very large self-pressure broadening coefficient, of the order of $140 \mathrm{MHz} m b a r^{-1}$. With the selected working pressure, the rotational lines of NEFA have a HWHM of $0.3-0.45 \mathrm{MHz}$ which is nicely adapted to measuring frequencies with high accuracy.

We found that NEFA partially decomposes since rotational lines of $\mathrm{HCN}, \mathrm{HC}_{3} \mathrm{~N}$, and $\mathrm{CH}_{3} \mathrm{CN}$ were detected during the experiment. Only $\mathrm{HCN}$, and at a very low level, was present in the chamber before NEFA was injected. The observed lines of these species indicate that less than $20 \%$ of the NEFA molecules decomposed.

The three dipole moment components of the molecule, $\mu_{a}$, $\mu_{b}$, and $\mu_{c}$, are non-zero (Ohba et al. 2005; Vaquero-Vara et al.
2017), which will produce a large number of rotational transitions for a gas at ambient temperature. Consequently, we performed two frequency switching observations with different frequency throws, $\Delta v_{\mathrm{FS}}$, of 37 and $25 \mathrm{MHz}$. In this way, we were able to account for possible contamination of the emission lines by the absorption features produced during the folding process. These contaminating features are placed at $\pm \Delta v_{\mathrm{FS}}$ of each emission feature, and will thus appear at different frequencies in the two observations. The data with $\Delta v_{\mathrm{FS}}=37 \mathrm{MHz}$ have an integration time of $29 \mathrm{~h}$ per $W$ sub-band, while the spectrum with $\Delta v_{\mathrm{FS}}=25 \mathrm{MHz}$ has a total integration time of $59 \mathrm{~h}$ per $W$ subband and has been used as reference data for frequency measurements. It is the longest stand-alone integration performed to date with the GACELA set-up. The whole spectrum of the $Q$ and $W$ bands has been analysed and a baseline has been removed by frequency ranges of $20-30 \mathrm{MHz}$ depending on the density of lines. The less sensitive spectrum obtained with $\Delta v_{\mathrm{FS}}=37 \mathrm{MHz}$ is used only when the lines in the reference data are contaminated by absorption features, and also to check for line blendings, and, in some cases, baseline effects.

Figure 20 shows different frequency domains of the $W$-band spectrum. The bottom panels show two selected lines to emphasize the high sensitivity reached in this experiment $(\simeq 1 \mathrm{mK})$. NEFA exhibits indeed a very dense spectrum in the $W$ band, with more than 100 lines per GHz. In addition to the fact that the molecule has $a$-, $b$-, and $c$-type transitions (Ohba et al. 2005; Vaquero-Vara et al. 2017), there are several low-lying vibrational excited states (Bermúdez et al., in prep.), and other conformers, trans - ap and cis - ap, that are 137 and $482 \mathrm{~cm}^{-1}$ above the trans $-s c$ ground state conformer (Vaquero-Vara et al. 2017) that will contribute to the spectrum. The observed intensities are very low due to the large pressure broadening of the rotational lines of the molecule (which imposes a rather low working pressure of $3 \times 10^{-3} \mathrm{mbar}$; see above), to the large values of the rotational and vibrational partition functions of the molecule $\left(Q_{\text {rot }}(300 \mathrm{~K}) \simeq 8.5 \times 10^{4} ; Q_{\text {vib }}(300 \mathrm{~K}) \simeq 13.1\right.$, $\left.Q_{\text {conf }}(300 \mathrm{~K}) \simeq 1.47\right)$, and to its partial decomposition when in contact with the walls of the chamber. Nevertheless, the final noise level reached is $\sim 1-2 \mathrm{mK}$ with peak line intensities varying from 10 up to $80 \mathrm{mK}$, i.e. $S / N>10$ (see bottom right panel of Fig. 20). We focus in this paper on the ground state of NEFA. A detailed analysis of the spectrum of the molecule, its vibrationally excited states, and its conformers is under preparation and will be published elsewhere (Bermúdez et al., in prep.).

The uncertainty for most frequency determinations in the spectrum of NEFA is of a few $\mathrm{kHz}$ in view of the high $\mathrm{S} / \mathrm{N}$ of the data (see Fig. 20). However, we have assigned an uncertainty of $10 \mathrm{kHz}$ for all lines showing a single component and intensity larger than $20 \mathrm{mK}(S / N \geq 20)$. Unlike linear molecules with a nitrogen atom, for which hyperfine splittings rapidly collapse, symmetric or asymmetric tops can show significant hyperfine splitting even for high- $J$ and high- $K_{a}$ transitions. As an example, the bottom panel of Fig. 10 shows the hyperfine structure of $K=3$ component of the $J=6 \rightarrow 5$ rotational transition of $\mathrm{CH}_{3} \mathrm{CN}$. The $K=0$ and 1 components of the same transition do not show any measurable splitting, while the $K=2$ exhibits an emerging shoulder (clearly seen in that figure) due to its hyperfine structure. Using the diagonal elements of the ${ }^{14} \mathrm{~N}$ quadrupole coupling tensor in NEFA (Ohba et al. 2005), we have computed the expected hyperfine splitting of all lines in the $W$ band. Most of the lines we have identified have the hyperfine splitting concentrated around $\pm 10 \mathrm{kHz}$ of the central frequency. However, several lines present three hyperfine components with similar intensities. Two of these components are at practically 

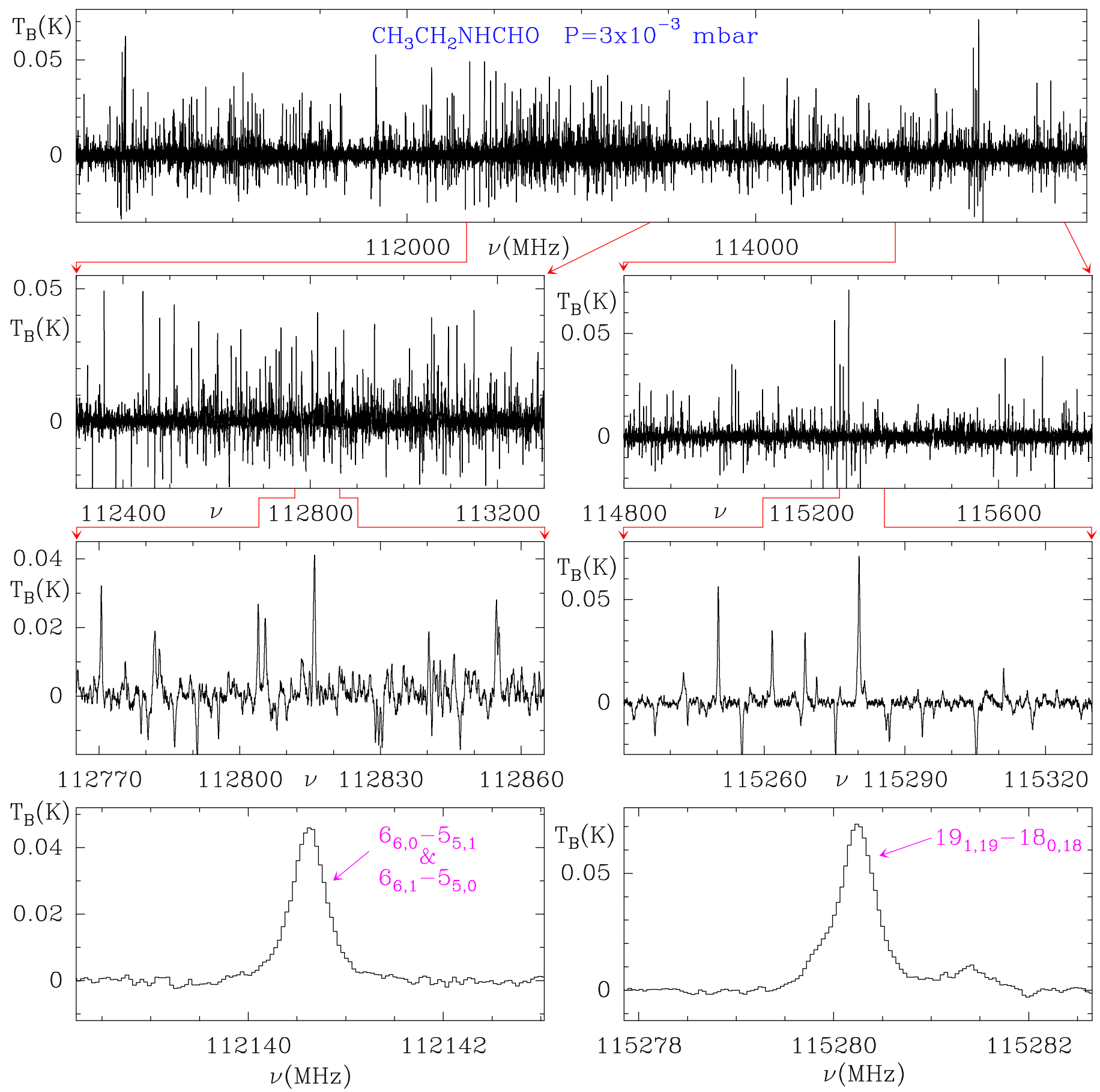

Fig. 20. Observed spectrum of $\mathrm{N}$-ethylformamide between 110.1 and $115.9 \mathrm{GHz}$. The total observing time per $W$ sub-band was $59 \mathrm{~h}$. The observing procedure was frequency switching with a throw of $25 \mathrm{MHz}$. The pressure in the chamber was $3 \times 10^{-3} \mathrm{mbar}$. The intensity scale is in $\mathrm{K}$ and the frequency scale in MHz. The different panels show different magnifications of the data of the upper panel. The two lines at the bottom have a linewidth of $\simeq 0.4 \mathrm{MHz}$. The $19_{1,19}-18_{0,18}$ transition shows a shoulder at lower frequencies produced by a line arising from one of the low-lying vibrationally excited states or from the conformers trans-ap or cis-ac (the expected hyperfine splitting for this transition is $\simeq 15 \mathrm{kHz}$ ).

the same frequency, while the third component is always on the opposite side producing a global splitting of 60-100 kHz. Hence, some of the observed lines could have a two-component structure, with intensity ratio 1:2, with a strong feature accompanied by less intense shoulder (a factor of two lower), either left or right of the central frequency. Many other lines also show a complex profile due to blending with lines coming from the vibrationally excited states and the other conformers of NEFA (see, for example, the bottom right panel of Fig. 20). We have fitted in most cases one single Gaussian profile to the observed lines. However, when the line profile exhibits two components we fitted two Gaussians. Depending on the degree of overlap between these components, the assigned uncertainty varies between 20 and $80 \mathrm{kHz}$. When two components were obvious in the line profile, the feature assigned to NEFA was based on the expected intensity of the transition (determined with the MADEX code; Cernicharo 2012), and by comparison with the intensity of other nearby lines of NEFA. 
Table 2. Rotational and distortion constants of $\mathrm{CH}_{3} \mathrm{CH}_{2} \mathrm{NHCHO}$.

\begin{tabular}{lrr}
\hline \hline $\begin{array}{l}\text { Molecular } \\
\text { constants }\end{array}$ & \multicolumn{1}{c}{$\begin{array}{c}\text { Values } \\
(\mathrm{MHz})\end{array}$} & $\begin{array}{c}\text { Previous work } \\
(\mathrm{MHz})\end{array}$ \\
\hline$A$ & $9904.836765(905)$ & $9904.8373(6)$ \\
$B$ & $3521.099684(209)$ & $3521.0995(2)$ \\
$C$ & $2984.979533(186)$ & $2984.9808(2)$ \\
$\Delta_{J}$ & $9.376237(349) \times 10^{-03}$ & $9.3859(53) \times 10^{-03}$ \\
$\Delta_{J K}$ & $-6.297555(282) \times 10^{-02}$ & $-6.2957(31) \times 10^{-02}$ \\
$\Delta_{K}$ & $1.659087(181) \times 10^{-01}$ & $1.6587(15) \times 10^{-01}$ \\
$\delta_{J}$ & $3.223793(197) \times 10^{-03}$ & $3.2310(14) \times 10^{-03}$ \\
$\delta_{K}$ & $1.044634(260) \times 10^{-02}$ & $1.0116(63) \times 10^{-02}$ \\
$\Phi_{J}$ & $-2.57722(178) \times 10^{-07}$ & \\
$\Phi_{J K}$ & $3.47486(457) \times 10^{-06}$ & \\
$\Phi_{K J}$ & $-1.82342(324) \times 10^{-05}$ & \\
$\Phi_{K}$ & $3.7193(109) \times 10^{-05}$ & \\
$\phi_{J}$ & $-1.06085(209) \times 10^{-07}$ & \\
$\phi_{J K}$ & $-3.6172(370) \times 10^{-07}$ & \\
$\phi_{K}$ & $3.8330(475) \times 10^{-06}$ & \\
$L_{J J K}$ & $2.3014(161) \times 10^{-10}$ & \\
$L_{K K J}$ & $4.201(160) \times 10^{-09}$ & \\
$l_{J}$ & $-5.6239(906) \times 10^{-12}$ & \\
$l_{K}$ & $1.1741(316) \times 10^{-08}$ & \\
$P_{J J K}$ & $1.5318(177) \times 10^{-13}$ & \\
$P_{J K}$ & $-8.165(138) \times 10^{-13}$ & \\
$p_{J}$ & $-4.848(206) \times 10^{-16}$ & \\
$p_{J J K}$ & $-3.732(121) \times 10^{-14}$ & \\
$p_{J K}$ & $2.0599(248) \times 10^{-12}$ & \\
\hline & &
\end{tabular}

Notes. All constants are in MHz. Numbers in parentheses represent the derived uncertainty $(1 \sigma)$ of the parameter in units of the last digit. The number of independent lines is 616 , of which 64 correspond to unresolved doublets. ${ }^{(a)}$ Previous constants derived by Ohba et al (2005).

Ohba et al. (2005) have found that the lines they observed in the microwave domain do not show any evidence of splitting due to internal rotation of the methyl group, $\mathrm{CH}_{3}$, and derived a lower limit of $1000 \mathrm{~cm}^{-1}$ to the barrier height of this internal rotation which is compatible with the estimated value derived from their ab initio calculations $\left(1400 \mathrm{~cm}^{-1}\right)$. Hence, no internal rotation splittings are expected for the rotational transitions of this molecule.

For the assignment of the rotational lines of the ground state of NEFA we proceeded using the rotational and distortion constants derived by Ohba et al. (2005). We started the search for the strongest predicted lines having the lowest possible $J$ and $K_{a}$ within the $Q$ and $W$ bands. The $b$-type transition $19_{1,19-}$ $18_{0,18}$ was predicted several $\mathrm{MHz}$ away from the strong line shown in the bottom right panel of Fig. 20. The other strong line close to this feature (see the spectrum between 115230 and $115330 \mathrm{MHz}$ in the second panel from the bottom, right side, in Fig. 20) was assigned to the $b$-type transition $19_{0,19}-18_{1,18}$ and two features placed between the two lines, and with a $50 \%$ intensity as expected, were easily assigned to the $a$-type transitions $19_{1,19}-18_{1,18}$ and $19_{0,19}-18_{0,18}$. A complete series of lines of the $b$-type Q branch $K_{a}=4 \rightarrow 3(\Delta J=0, J=14$ to 39) were also assigned. These lines were fitted using the FITWAT code (Cernicharo et al. 2018a) to derive a new series of rotational and distortion constants; these new constants were used for new frequency predictions, allowing the assignment of new lines in the $Q$ and $W$ bands. Several sets of $a$-type $\left(K_{a}=0-14\right)$ and $b$-type (up to $K_{a}=15$ ) were then identified and fitted; $c$-type transitions are too weak to be assigned within the sensitivity of the data.

The final set of data for the ground state consists of 616 lines assigned in the $Q$ and $W$ bands with $J(\max )=54$ and $K_{a}(\max )=15$. Of these lines, 64 are unresolved $K$-doublets which gives a total of 552 independent frequency measurements. Table C. 2 gives the frequencies and their uncertainties for all these lines. The inclusion of high- $J$ lines requires the incorporation of high-order distortion constants to fit the data within the observed accuracy, which is a consequence of the highly floppy nature of the molecule (Bermúdez et al., in prep.). The lines have been fitted to 24 rotational and distortion constants of a Watson Hamiltonian (Watson 1977) in the $A$ reduction and $I^{r}$ representation using the FITWAT code (Cernicharo et al. 2018a) which produces a FORTRAN routine that can be directly incorporated into the MADEX code (Cernicharo 2012). The standard deviation of the fit is $30 \mathrm{kHz}$ and the weighted value is 0.85 ; we also used the SPFIT/SPCAT code (Pickett 1991) and obtained exactly the same results.

The derived constants with their uncertainties are given in Table 2. The agreement of our rotational and quartic distortion constants with those derived by Ohba et al. (2005) is excellent. The improvement of the uncertainty of the present quartic distortion constants by a factor 20-30 should be noted. The highest order constants are strongly correlated, but are essential to fit the observed frequencies due to the floppiness of the molecule. They permit predicting the spectrum up to $200 \mathrm{GHz}$, and even up to $300 \mathrm{GHz}$ for low values of $K_{a}$. Caution should be applied to frequency predictions for $J>55, K_{a}>15$.

\subsection{Search for $N$-ethylformamide in space}

Using the rotational constants of Table 2 and the MADEX code Cernicharo (2012) we searched for NEFA in cold and warm molecular clouds. The adopted dipole moments are those calculated by Vaquero-Vara et al. (2017) $\left(\mu_{a}=2.2 \mathrm{D}, \mu_{b}=2.9 \mathrm{D}\right.$, and $\left.\mu_{c}=1.0 \mathrm{D}\right)$.

The cold dense cores TMC1-1 and B1-b have been studied in detail through systematic observations at all wavelengths. In particular we used our data at $3 \mathrm{~mm}$ (Marcelino et al. 2005, 2007, 2009, 2010; Daniel et al. 2013; Fuente et al. 2016; Cernicharo et al. 2012, 2013，2014，2018b) to search for NEFA towards these two objects. We selected two practically unresolved doublets, $5_{51}-4_{40} / 5_{50}-4_{41}$ at $92363.100 \mathrm{MHz}$ and $6_{61}-5_{50} / 6_{60}-5_{51}$ at $112140.638 \mathrm{MHz}$, to increase the chance of detecting the species. For B1-b we adopted a kinetic temperature of $12 \mathrm{~K}$ (Cernicharo et al. 2012) and a linewidth at half intensity of $0.5 \mathrm{~km} \mathrm{~s}^{-1}$. None of these lines are detected in our data, which provides a $3 \sigma$ upper limit of the column density of NEFA of $\simeq 10^{12} \mathrm{~cm}^{-2}$. For TMC1-1 we adopted a kinetic temperature of $10 \mathrm{~K}$ obtaining a similar upper limit to the column density of NEFA. These results are not totally unexpected since formamide itself has not been detected towards these sources.

We also searched for NEFA in two prototypical high-mass star-forming regions, Orion KL and Sgr B2, using the ALMA Science Verification (SV) data of Orion KL between 213.7 and $246.6 \mathrm{GHz}$ (for observations and data reduction, see e.g. Tercero et al. 2018) and the IRAM $30 \mathrm{~m} 3 \mathrm{~mm}$ survey of Sgr B2 provided by Belloche et al. (2013). The high angular resolution of the ALMA data $\sim 2.00^{\prime \prime} \times 1.50^{\prime \prime}$ allows us to concentrate the search in the emission peak of the related species $\mathrm{NH}_{2} \mathrm{CHO}$ and $\mathrm{CH}_{3} \mathrm{NCO}$ (see Cernicharo et al. 2016). Nevertheless, owing to the high frequencies of these data, we restricted the search 
Table 3. N-ethylformamide column densities.

\begin{tabular}{|c|c|c|}
\hline & $\begin{array}{l}\text { Orion KL }{ }^{(c)} \\
\text { (ALMA SV) }\end{array}$ & $\begin{array}{l}\text { Sgr B2 }{ }^{(d)} \\
(\text { IRAM } 30 \mathrm{~m})\end{array}$ \\
\hline Coordinates & $\alpha=5^{\mathrm{h}} 35^{\mathrm{m}} 14.5^{\mathrm{s}}$ & $\alpha=17^{\mathrm{h}} 47^{\mathrm{m}} 20.0^{\mathrm{s}}$ \\
\hline$(\mathrm{J} 2000.0)$ & $\delta=-05^{\circ} 22^{\prime} 33.0^{\prime \prime}$ & $\delta=-28^{\circ} 22^{\prime} 19.0^{\prime \prime}$ \\
\hline $\mathrm{HPBW}^{(a)}\left({ }^{\prime \prime}\right)$ & $\sim 2.0 \times 1.5$ & $30-21$ \\
\hline Frequency ${ }^{(b)}(\mathrm{GHz})$ & $213.7-246.6$ & $80-115.5$ \\
\hline$v_{\mathrm{LSR}}\left(\mathrm{km} \mathrm{s}^{-1}\right)$ & 8.0 & 63.0 \\
\hline$\Delta v_{\text {FWHM }}\left(\mathrm{km} \mathrm{s}^{-1}\right)$ & 3.0 & 7.0 \\
\hline$d_{\text {sou }}\left({ }^{\prime \prime}\right)$ & 3.0 & 2.4 \\
\hline$T_{\text {rot }}(\mathrm{K})$ & 150 & 180 \\
\hline$N\left(\mathrm{NH}_{2} \mathrm{CHO}\right)\left(\mathrm{cm}^{-2}\right)$ & $3.0 \times 10^{15}$ & $1.3 \times 10^{18}$ \\
\hline$N\left(\mathrm{CH}_{3} \mathrm{NHCHO}\right)\left(\mathrm{cm}^{-2}\right)$ & $\leq 1.0 \times 10^{15}$ & $\leq 2.0 \times 10^{17}$ \\
\hline$N(\mathrm{NEFA})\left(\mathrm{cm}^{-2}\right)$ & $\leq 1.0 \times 10^{14}$ & $\leq 1.0 \times 10^{17}$ \\
\hline$N\left(\mathrm{NH}_{2} \mathrm{CHO}\right) / N\left(\mathrm{CH}_{3} \mathrm{NHCHO}\right)$ & $\geq 3$ & $\geq 6.5$ \\
\hline$N\left(\mathrm{NH}_{2} \mathrm{CHO}\right) / N(\mathrm{NEFA})$ & $\geq 30$ & $\geq 13$ \\
\hline
\end{tabular}

Notes. ${ }^{(a)} \mathrm{HPBW}$ (half power beam width) for observations with singledish telescope (IRAM 30m) and synthetic beam for the ALMA SV observations. ${ }^{(b)}$ Range of frequencies considered in the analysis. ${ }^{(c)}$ Physical parameters derived for the main spectral component of $\mathrm{NH}_{2} \mathrm{CHO}$ by Cernicharo et al. (2016). ${ }^{(d)}$ Physical parameters derived for the main spectral component of $\mathrm{NH}_{2} \mathrm{CHO}$ by Belloche et al. (2013).

for NEFA to lines with $K_{\mathrm{a}} \leq 5$ in Orion $\mathrm{KL}$ to ensure the line identification.

We did not find this species above the detection limit of both sets of data. To provide upper limits to its column density, we used MADEX to derive the synthetic spectrum of NEFA for both sources assuming the same physical parameters as those derived for $\mathrm{NH}_{2} \mathrm{CHO}$ by Cernicharo et al. (2016) and Belloche et al. (2013) in Orion KL and Sgr B2, respectively. Moreover, we searched for $\mathrm{CH}_{3} \mathrm{NHCHO}$ in these sources to constrain the relative abundances of these related species. The results are summarized in Table 3. It is worth noting that due to the high line blending in the spectra of the two sources, together with the large partition functions of NEFA and of $\mathrm{CH}_{3} \mathrm{NHCHO}$, the derived upper limits correspond to relatively large values for the column density. Consequently, they are not very constraining for chemical models.

\section{Conclusions}

In this paper we have shown the huge potential of broad-band, high spectral resolution, rotational spectroscopy using radio astronomical receivers and spectrometers as detecting devices. The sensitivity attained is similar to that obtained with the largest radio telescopes equipped with state-of-the-art receivers.

The GACELA set-up can be used for a large variety of experiments. Broad-band rotational spectroscopy, with its high instantaneous bandwidth, allows us to perform chemical experiments in which the appearance and disappearance of molecular species can help to explain astronomical observations of molecular emission. We have shown in Sect. 3 the enormous sensitivity we can reach in detecting polar species in a few seconds of observing time. Cold plasma experiments have been described in Sect. 4 showing the potential to generate molecular species in the gas phase and also by interaction of atoms and radicals with the walls of the chamber (see Sect. 4.5). The capability of the GACELA set-up to perform rotational spectroscopy of interesting molecular species has been shown in Sect. 5.1.

Broad-band rotational emission spectroscopy could be used for many other experiments with GACELA, or in other reactors, where monitoring of the gas phase composition of polar molecules is needed. In addition, the high spectral resolution and high sensitivity provided by our detection system could permit the identification of new molecular species produced in plasma or UV experiments and their search in space.

The molecular thermal emission measurements performed with the GACELA set-up permits us to derive accurate partial pressures for the gases filling the chamber (uncertainty below $3 \%$ for $S / N>30$ ).

We conclude that radio astronomical receivers such as those of the GACELA set-up can be used in the field of Laboratory Astrophysics in a large number of novel experiments.

Acknowledgements. We thank the European Research Council for funding support under Synergy Grant ERC-2013-SyG, G.A. 610256 (NANOCOSMOS). IT, VJH, and JLD acknowledge additional partial support from the Spanish State Research Agency (AEI) through grant FIS2016-77726-C3-1-P. JAMG, LM, and GS acknowledge additional partial support from the Spanish State Research Agency (AEI) through grant MAT2017-85089-c2-1R. We thank David López Romero for his help during the process of installation, commissioning, and cleaning of the chamber. We would like to thank Kremena Makasheva for the useful comments and suggestions during the experiments with Hexamethyldisiloxane. We would also like to thank Rosa Lebrón, Jesús Quintanilla, and Cristina Soria for providing us with the sample of N-ethylformamide. Sandra I. Ramírez acknowledges support from the FONCICYT under grant number 291842. Celina Bermúdez thanks the Spanish Ministerio de Ciencia Innovación y Universidades for the Juan de la Cierva grant FJCI-2016-27983.

\section{References}

Alexander, M. R., Jones, F. R., \& Short, R. D. 1997, J. Phys. Chem. B, 101, 3614 Atkinson, R., Baulch, D. L., Cox, R. A., et al. 1997, J. Chem. Phys. Ref. Data, 26, 1329

Basner, R., Foest, R., Schmidt, M., et al. 1998, Int. J. of Mass Spectrom., 176, 245

Bell, T. A., Cernicharo, J., Viti, S., et al. 2014, A\&A, 564, A114

Belloche, A., Müller, H. S. P., Menten, K. M., et al. 2013, A\&A, 559, A47

Belloche, A., Meshcheryakov, A. A., Garrod, R. T., et al. 2017, A\&A, 601, A49

Botta, L., Saladino, R., Bizzarru, B. M., et al. 2018, Adv. Space Res., 62, 2372

Carrasco, E., Jiménez-Redondo, M., Tanarro, I., \& Herrero, V. J. 2012, Plasma Phys. Control. Fusion, 54, 124019

Ceccarelli, C., Caselli, P., Fontani, F., et al. 2017, ApJ, 850, 176

Cernicharo, J. 1985, ATM a Code to Compute Atmospheric Opacity up to $1 \mathrm{THz}$, IRAM Internal Report

Cernicharo, J. 2012, in ECLA-2011: Proc. of the European Conference on Laboratory Astrophysics, eds. C. Stehl, C. Joblin, \& L. d'Hendecourt (Cambridge: Cambridge Univ. Press), EAS Pub. Ser., 251

Cernicharo, J., Marcelino, N., Roueff, E., et al. 2012, ApJ, 759, L43

Cernicharo, J., Tercero, B., Fuente, A., et al. 2013, ApJ, 771, L10

Cernicharo, J., Bailleux, S., Alekseev, E., et al. 2014, ApJ, 795, 40

Cernicharo, J., Kissiel, Z., Tercero, B., et al. 2016, A\&A, 587, L4

Cernicharo, J., Guélin, M., Agúndez, M., et al. 2018a, A\&A, 618, A4 Cernicharo, J., Lefloch, B., Agúndez, M., et al. 2018b, ApJ, 853, L22

Cueto, M., Cernicharo, J., Barlow, M. J., et al. 2014, AJ, 783, 2014

Daniel, F., Gérin, M., Roueff, E., et al. 2013, A\&A, 560, A3

DeMore, W. B., Sander, S. P., Golden, D. M., et al. 1997, JPL pub., 97-4, 1

Despax, B., Gaboriou, F., Caquineau, H., \& Makasheva, M. 2016, AIP Adv., 6, 105111

Doménech, J. L., Cueto, M., Herrero, V. J., et al. 2013, ApJ, 771, L11

Doménech, J. L., Drouin, B. J., Cernicharo, J., et al. 2016, ApJ, 853, L32

Frisk, U., Hagström, M., Ala-Laurinaho, J., et al. 2003, A\&A, 402, L27

Fuente, A., Cernicharo, J., Roueff, E., et al. 2016, A\&A, 593, A94

Higgins, R. D. 2011, PhD Lecture, NUI Maynooth, Ireland

Higgins, R. D., Teyssier, D., Pearson, J. C., et al. 2010, Twenty-First International Symposium on Space Terahertz Technology, National Radio Astronomy Observatory (NRAO), 2010, 390

Hüberts, H. W., Pavlov, S. G., Richter, H., \& Semenov, A. D. 2006, Appl. Phys. Let., 89, 061115

Juris, M., Coplen, T. B., Berglund, M., et al. 2016, Pure Appl. Chem., 88, 265

Koshelev, M., \& Tetryakov, M. 2009, J. Quant. Spectr. Rad. Transf., 110, 118

Leuther, A., Tessmann, A., Kallfass, I., et al. 2009, IEEE International Conference on Indium Phosphide and Related Materials, Newport Beach, CA, 2009, 188

Ligterink, N. F. W., Terwisscha van Scheltinga, J., Taquet, V., et al. 2018, MNRAS, 480, 3628 
J. Cernicharo et al.: Broad-band rotational spectroscopy for laboratory astrophysics

Malo, I., Gallego, J. D., Amils, R., et al. 2016, Technical Report IT-CDT 2016-6 (http://www1.oan.es/reports/doc/IT-CDT-2016-6.pdf)

Marcelino, N., Cernicharo, J., Roueff, E., et al. 2005, ApJ, 620, 308

Marcelino, N., Cernicharo, J., Agúndez, M., et al. 2007, ApJ, 665, L127

Marcelino, N., Cernicharo, J., Tercero, B., et al. 2009, ApJ, 690, L27

Marcelino, N., Brünken, S., Cernicharo, J., et al. 2010, A\&A, 516, A105

Martínez, L., Lauwaet, K., Santoro, G., et al. 2018, Sci. Rep., 8, 7250

Müller, H. S. P., Schlöder, F., Stutzki, J., \& Winnewisser, G. 2005, J. Mol. Struct., 742,215

Neumaier, P. F. X., Richter, H., Stake, J., et al. 2014, IEEE Trans. TeraHertz Sci. Tech., 4, 469

Ohba, K., Usami, T., Kawashima, Y., \& Hirota, E. 2005, J. Mol. Struct., 744, 815

Pardo, J. R., Cernicharo, J., \& Serabyn, E. 2001, IEEE Trans. Antennas and Propagation, 49, 1683

Pickett, H. M. 1991, J. Mol. Spectr., 148, 371

Pickett, H. M., Poynter, R. L., Cohen, E. A., et al. 1998, JQSRT, 60, 883
Pardo, J. R., Pagani, L., Gerin, M., \& Prigent, C. 1995, J. Quant. Spectr. Rad. Transf., 54, 931

Ren, Y., Hovenier, J. N., Higgins, R., et al. 2010, Appl. Phys. Let., 97, 161105 Sagan, C., \& Khare, B. 1979, Nature, 277, 102

Tanarro, I., Herrero, V. J., Carrasco, E., \& Jiménez-Redondo, M. 2011, Vacuum, 85,1120

Tanarro, I., Alemán, B., de Vicente, P., et al. 2018, A\&A, 609, A15

Tercero, B., Cuadrado, S., López, A., et al. 2018, A\&A, 620, L6

Teyssier, D., Dartois, E., Crussaire, J. P., et al. 2004, Gopal Narayanan., 2004, 306

Tolls, V., Melnick, G. J., Ashby, M. L., et al. 2004, ApJS, 152, 137

Vaquero-Vara, V., Alstadt, V., Sewatsky, T. P., et al. 2017, J. Mol. Spectr., 335, 102

Watson, J. K. G. 1977, in Vibration Spectra and Structure, ed. J. Durig (Amsterdam: Elsevier), 6, 1

Wehres, N., Maßen, J., Borisov, K., et al. 2018a, PCCP, 20, 5530

Wehres, N., Heyne, B., Lewen, F., et al. 2018b, Pro. Int. Astron. Union, 13, 332 


\section{Appendix A: Contribution of the different elements of the GACELA set-up to the system temperature}
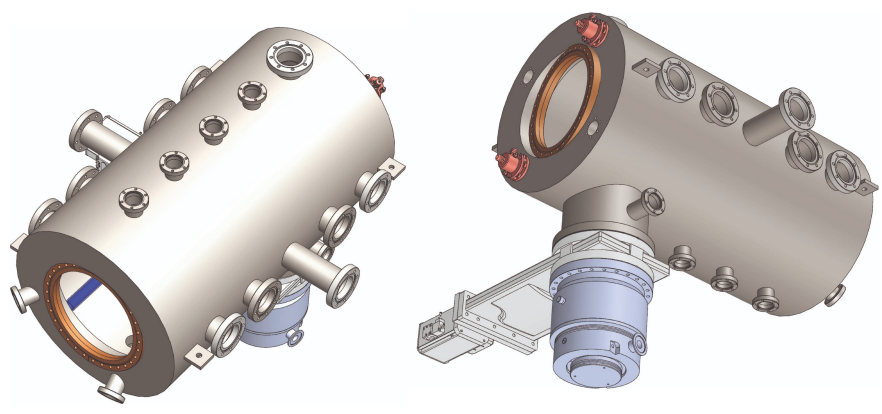

Fig. A.1. Two views of the chamber of GACELA, from the top (left) and the bottom (right). The turbomolecular pump is connected to a DN $250 \mathrm{CF}$ flange near the left edge (see right panel).

In addition to the receiver noise we have to consider the effects introduced in the system temperature by the chamber of GACELA, the RF coil inside it, and the Teflon ${ }^{\mathrm{TM}}$ windows. Each of these elements can intercept the radio beam from the receivers, and thus could contribute to the total system temperature that limits the final sensitivity of the GACELA experimental set-up. The optical diagram of GACELA is shown in Fig. 1. Figure A.1 shows the design of the chamber. A picture showing the coil for the RF generator, which was designed to surround the radio beam of the receivers, is shown in Fig. A.2.

In order to derive the contribution of each of these elements we followed several steps. First, the receiver noise temperature was derived using an absorber at ambient temperature and a cold load at the temperature of liquid nitrogen. These absorbers were placed in front of the receiver window. The derived noise temperatures in $\mathrm{Q}$ and $\mathrm{W}$ bands are shown in the top panels of Figs. A.3 and A.4, respectively. In addition to the measure of the receiver noise temperatures, these absorbers were used to derive the temperature of the internal and background loads which are connected to a $15 \mathrm{~K}$ cold stage.

We found that the background cold load was at a temperature close to $20 \mathrm{~K}$ for $Q$ and $W$ bands. The internal cold load was found to present an equivalent noise temperature 5-10 K warmer than the background cold load. However, its physical temperature was not accurately known since the temperature sensor was attached to the cold stage of the refrigerator and was not in direct contact with the microwave absorber (see below).

Once the receiver temperature and that of the internal and background loads were derived, we placed the GACELA chamber (without the RF coil, and without the Teflon ${ }^{\mathrm{TM}}$ windows) in its estimated geometrical position (see Fig. 2). A new set of measurements of the system temperature was performed. The RF coil was then installed, and finally the Teflon ${ }^{\mathrm{TM}}$ windows. In all these measurements of system performance the ambient temperature load and one load immersed in liquid $\mathrm{N}_{2}$ were used. Figures A. 3 and A.4 show the different sets of measurements.

In $W$ band, when the chamber is in the receiver path (without the RF coil and Teflon ${ }^{\mathrm{TM}}$ windows), the system temperature is very close to $T_{\text {rec }}+T_{\text {cold_background }}$, i.e. the chamber itself does not degrade the receiver performance (the red plot in middle panel of Fig. A.4 coincides with the black plot; it also coincides with the blue plot as discussed below). However, in the $Q$ band there is a

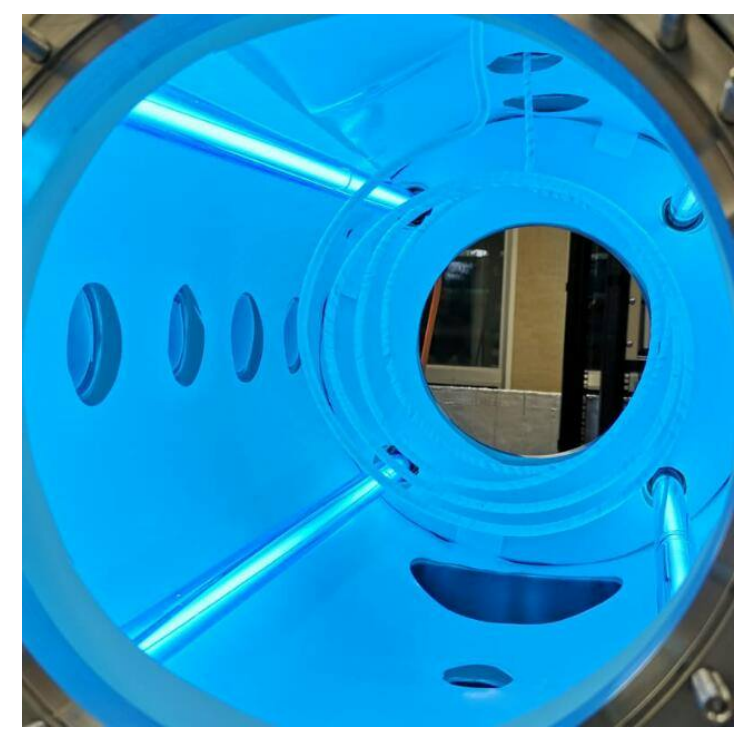

Fig. A.2. Photograph of the interior of the reactor, showing the UV lamps, the Teflon ${ }^{\mathrm{TM}}$ lining of the walls, and the RF coil.

contribution of 5-7 K to the system temperature by the chamber, as seen by comparing the red and black plots in the middle panel of Fig. A.3.

Inserting the RF coil in the chamber has a contribution of $1-2 \mathrm{~K}$ to the system temperature in the $Q$ band (compare the blue and red plots in the middle panel of Fig. A.3). In the $W$ band the RF coil does not contribute to the system temperature (the black, red, and blue plots coincide in the middle panel of Fig. A.4).

Finally, inserting the windows has a strong effect on both bands. Although the Teflon ${ }^{\mathrm{TM}}$ windows were designed to transmit at the best of the two bands, we measured a strong increase in the system temperature above $36 \mathrm{GHz}$ in the $Q$ band. The largest contribution is found around $43 \mathrm{GHz}(15 \mathrm{~K})$.

In the $W$ band, inserting the Teflon ${ }^{\mathrm{TM}}$ windows produces an increase in the system temperature of $15-20 \mathrm{~K}$ around $100 \mathrm{GHz}$. In both bands the windows produce a strong dependence of the system temperature on frequency.

The internal cold load is used for standard ON/OFF observations (see Sect. 2.10). Hence, it is important to measure its temperature difference with the background cold load, $\Delta T=$ $T$ (BCL) $-T$ (ICL) across the $Q$ and $W$ bands. The bottom panel of Figs A.3 and A.4 shows the effect of the different elements of the chamber in $\Delta T$.

In $W$ band, the ICL is warmer by $8-9 \mathrm{~K}$ than the BCL across the band when the chamber is inserted in the path of the receiver. This temperature difference decreases to 6-7 K when the RF coil is inserted. Finally, when the Teflon ${ }^{\mathrm{TM}}$ windows are inserted $\Delta \mathrm{T}$ shows a strong sinudoidal behaviour with frequency. The internal cold load is still warmer than the background cold load by $3-5 \mathrm{~K}$ up to $90 \mathrm{GHz}$. Above this frequency it becomes colder than the background load. A similar behaviour is found for the $Q$ band (see bottom panel of Fig. A.3).

When frequency switching observations are performed, the frequency throw is a few tens of $\mathrm{MHz}$ and all the observations are carried out with the background cold absorber. Therefore, we can assume the same temperature for the absorber at the two observing frequencies. 
J. Cernicharo et al.: Broad-band rotational spectroscopy for laboratory astrophysics

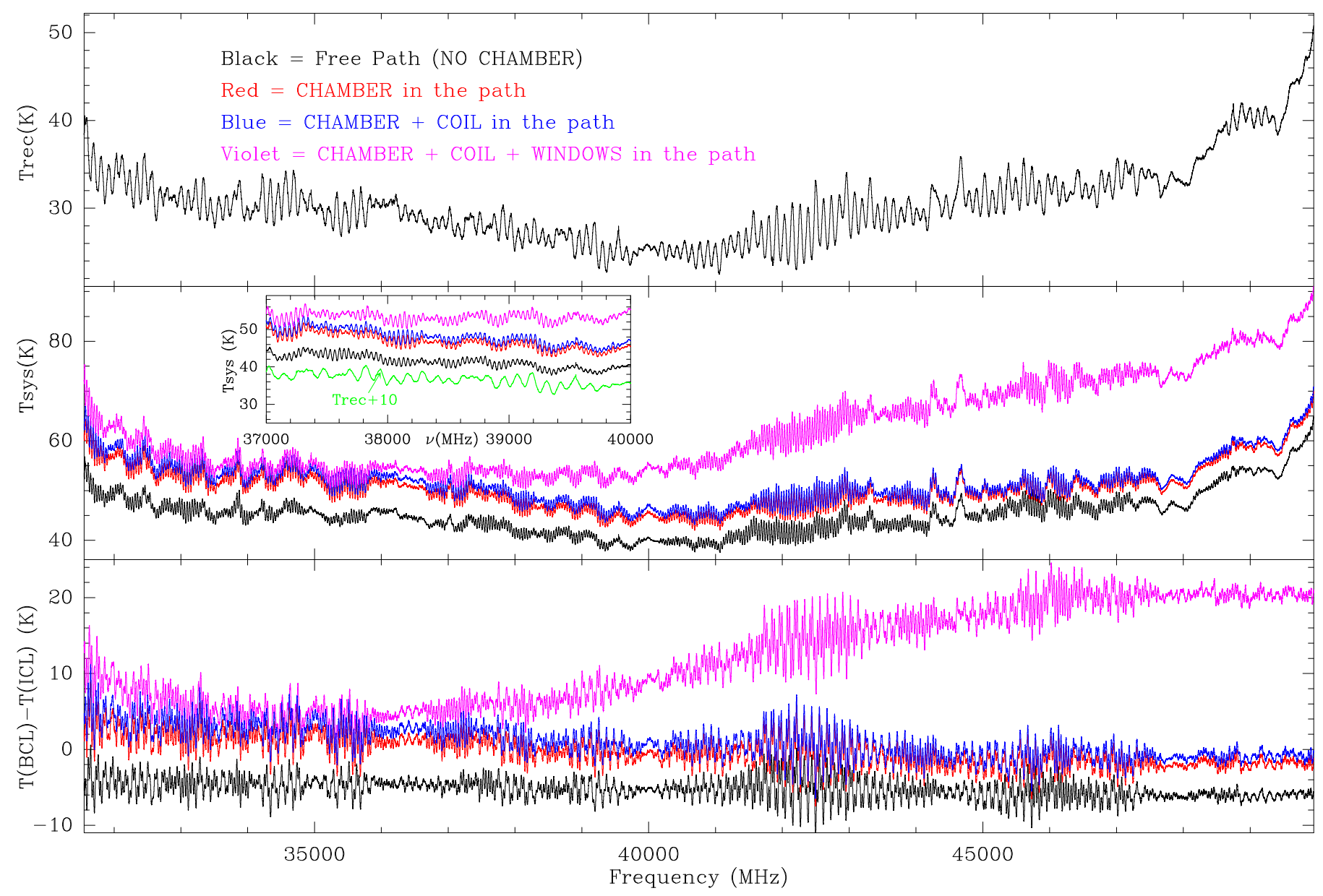

Fig. A.3. Top: receiver noise temperature in the $Q$ band derived from two absorbers at ambient and liquid $\mathrm{N}_{2}$ temperatures. Middle: system temperature (receiver + background cold load) when different elements of the system are inserted in the beam path of the receiver: free path (black), chamber (red), chamber plus RF coil (blue), and chamber plus RF coild plus Teflon ${ }^{\mathrm{TM}}$ windows (violet). The inset shows a zoom-in on frequency with the receiver temperature (top panel; free path) shifted by $10 \mathrm{~K}$ in cyan. Bottom: temperature difference between background (BCL) and internal (ICL) cold loads. Colours correspond to the same conditions as those indicated in the top panel. The receiver noise, system temperature, and the cold load temperature differences are in $\mathrm{K}$. 


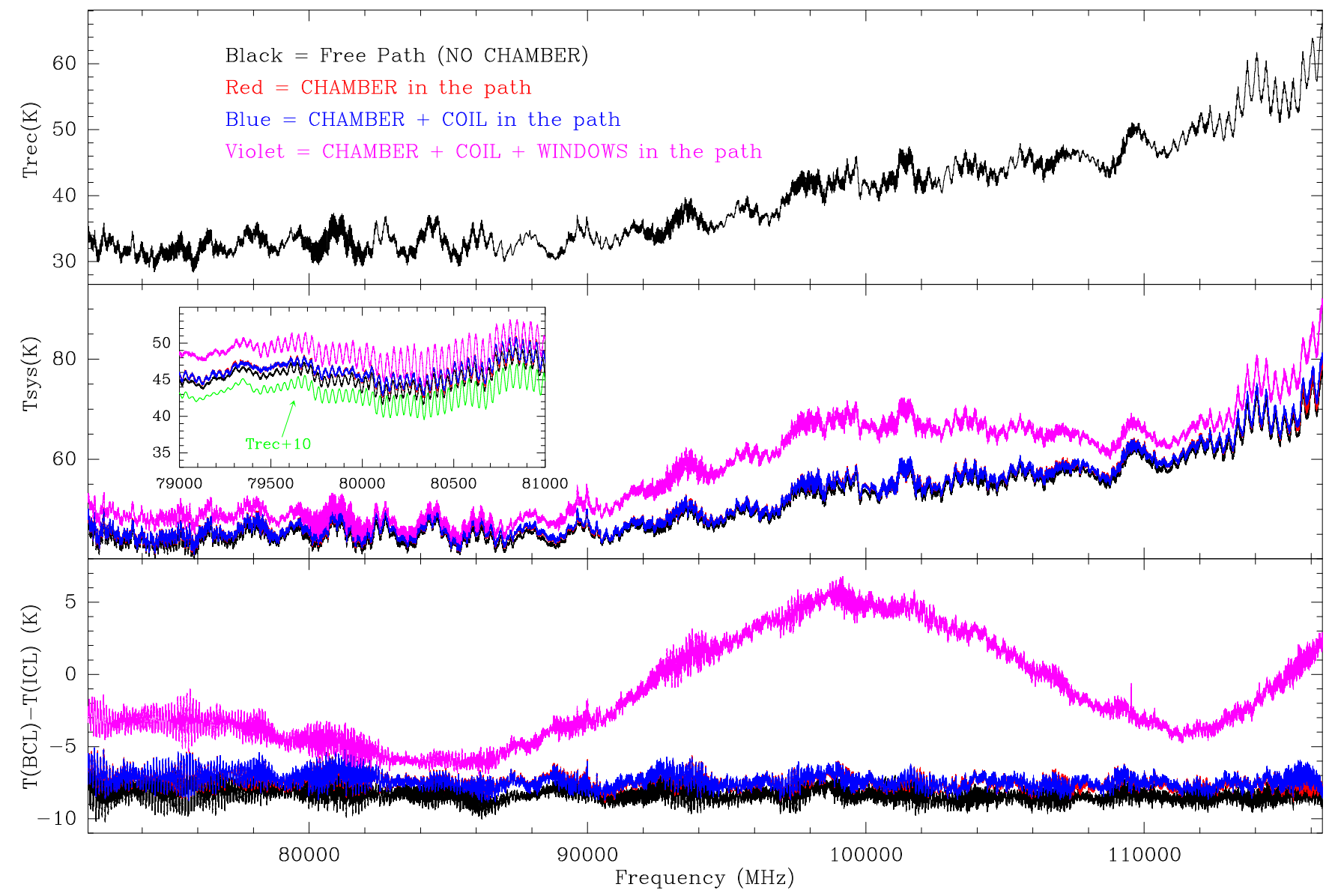

Fig. A.4. Same as for Fig. A.3, but for the $W$ band. 


\section{Appendix B: Absolute data calibration}

Details on the calibration of the data are given by Tanarro et al. (2018) for the protoype of the chamber. Here we reproduce the basic equations adapted to the final chamber and system components. We can assume as a first approximation that the windows of the cell are transparent at microwave wavelengths (see, however, Appendix A). Opposite to the receiver, behind the cell, there is a black body at temperature $T_{\mathrm{c}}$ (background cold load, see Fig. 2).

The gas in the cell is at temperature $T_{\mathrm{g}}$, with a volume density high enough to maintain it under thermal equilibrium conditions, i.e. all rotational levels are populated following a Boltzmann distribution at a single rotational temperature, which is identical to the kinetic temperature of the gas. As frequencies in our experiment are below $116.6 \mathrm{GHz}$ and the physical temperature of the cell is $\sim 300 \mathrm{~K}$, it results that $h v / K T<0.02$ ( $h$ and $K$ are the Planck and Boltzmann constants respectively). Hence, we can assume the Rayleigh-Jeans approximation to the line radiant flux $I_{v}$ and use a brightness temperature instead, $T_{B}$, so that $I_{v}(T)=2 v^{2} K T_{B} / c^{2}$.

The line intensity, or temperature, detected by a receiver in front of the cell at frequency $v$ (assumed to be close to the central frequency $v_{\mathrm{ul}}$ of the molecular transition $u \rightarrow l$ ) can be written as

$T_{B}^{\mathrm{ON}}(v)=T_{\mathrm{c}} e^{-\tau\left(v, T_{\mathrm{g}}\right)}+T_{\mathrm{g}}\left(1-e^{-\tau\left(v, T_{\mathrm{g}}\right)}\right)=\left(T_{\mathrm{c}}-T_{\mathrm{g}}\right) e^{-\tau\left(v, T_{\mathrm{g}}\right)}+T_{\mathrm{g}}$,

where $\tau\left(v, T_{\mathrm{g}}\right)$ is the gas opacity integrated along the cell's length at frequency $v$ and temperature $T_{\mathrm{g}}$. The line opacity depends on the molecule's dipole moment, the total number of molecules along the path $(90 \mathrm{~cm}$ in our case), the frequency, and the temperature (through the partition function), and has been analysed by Tanarro et al. (2018). When $T_{\mathrm{c}}=T_{\mathrm{g}}$, there is a lack of contrast and therefore the signal detected will not display any spectral signature: $T_{B}(v)=T_{\mathrm{g}}$. In this consideration we have neglected the emissivity and absorption of the cell's windows. When the receiver observes through an empty cell (or in frequency switching mode) the detected signal is

$T_{B}^{\mathrm{OFF}}(v)=T_{\mathrm{c}}$

The signal $T_{B}^{\mathrm{ON}-\mathrm{OFF}}(v)$, obtained by the standard ON-OFF or frequency switching techniques in radioastronomy, is given by

$T_{B}^{\mathrm{ON}-\mathrm{OFF}}(v)=\left(T_{\mathrm{g}}-T_{\mathrm{c}}\right)\left(1-e^{-\tau\left(v, T_{\mathrm{g}}\right)}\right)$.

If $T_{\mathrm{g}}>T_{\mathrm{c}}$ the spectral lines will be observed in emission. If $T_{\mathrm{g}}<T_{\mathrm{c}}$ they will be observed in absorption. For practical purposes we can assume $T_{\mathrm{c}}=20 \mathrm{~K}$ and $T_{\mathrm{g}}=295 \mathrm{~K}$. In the optically thick case, i.e. $\tau \gg 1, T_{B}=275 \mathrm{~K}$. In the optically thin case, $\tau \ll 1, T_{B}=275 \tau\left(v, T_{\mathrm{g}}\right)$. In the above equations we have assumed that the air is practically transparent at the wavelengths of the experiment. However, $\mathrm{O}_{2}$ has one cluster of absorption lines in the range $50-70 \mathrm{GHz}$ and another centred at $118.75 \mathrm{GHz}$. Water has a weak absorption feature at $22 \mathrm{GHz}$. Hence, the air path in this experiment should be maintained as short as possible. Nevertheless, the absorption produced by the air can be corrected using the ATM model (Cernicharo 1985; Pardo et al. 2001).

A trickier problem would be the absorption by the material used for the cell windows. As, in general, that absoption will be not negligible, we can generalize Eq. (B.1) as

$$
\begin{array}{r}
T_{B}^{O N}(v)=\left[\left\{T_{\mathrm{c}} e^{-\kappa_{\nu}}+T_{\mathrm{w}}\left(1-e^{-\kappa_{\nu}}\right)\right\} e^{-\tau\left(v, T_{\mathrm{g}}\right)}+\right. \\
\left.+T_{\mathrm{g}}\left(1-e^{-\tau\left(v, T_{\mathrm{g}}\right)}\right)\right] e^{-\kappa_{\nu}}+T_{\mathrm{w}}\left(1-e^{-\kappa_{\nu}}\right),
\end{array}
$$

where $T_{\mathrm{w}}$ is the temperature of the windows (in principle identical to $T_{\mathrm{g}}$ ), and $\kappa_{v}$ is the opacity of the windows at frequency $v$. The first term represents the emission of the cold load attenuated by the first window. The second term represents the emission of the first window. Both terms are attenuated by the gas in the cell. The third term represents the emission of the gas. Finally, the last window attenuates all the previous terms and emits itself (last term). In the case of an empty cell, we get the OFF signal

$T_{B}^{\mathrm{OFF}}(v)=\left[T_{\mathrm{c}} e^{-\kappa_{v}}+T_{\mathrm{w}}\left(1-e^{-\kappa_{v}}\right)\right] e^{-\kappa_{v}}+T_{\mathrm{w}}\left(1-e^{-\kappa_{v}}\right)$,

and the detected ON-OFF signal will be

$T_{B}^{\mathrm{ON}-\mathrm{OFF}}(v)=\left[T_{\mathrm{g}}-T_{\mathrm{c}} e^{-\kappa_{v}}-T_{\mathrm{w}}\left(1-e^{-\kappa_{v}}\right]\left(1-e^{-\tau\left(v, T_{\mathrm{g}}\right)}\right) e^{-\kappa_{v}}\right.$

The accuracy of the calibration can be degraded by the atmosphere (see above), the uncertainty in the transmission of the cell windows, as well as instrumental baseline effects. The short atmospheric path involved in the experiment ensures that the atmosphere has a negligible effect on the calibration of the observations. Taking these factors into account, we estimate the intensity uncertainties in our measurements to be below $3 \%$.

\section{Appendix C: Observed spectra in $Q$ and W bands, and line lists}

Selected spectra from the different experiments performed in this work are shown if Figs. 7, 8, 10-12, and C.2. In this section we show the whole $Q$ - and/or $W$-band spectra of some of the experiments discussed in the text. In addition, $\mathrm{a} \mathrm{SO}_{2}$ line list of some of the performed experiments and the observed lines of the ground state of NEFA $\left(\mathrm{CH}_{3} \mathrm{CH}_{2} \mathrm{NHCHO}\right)$ are also given here. 

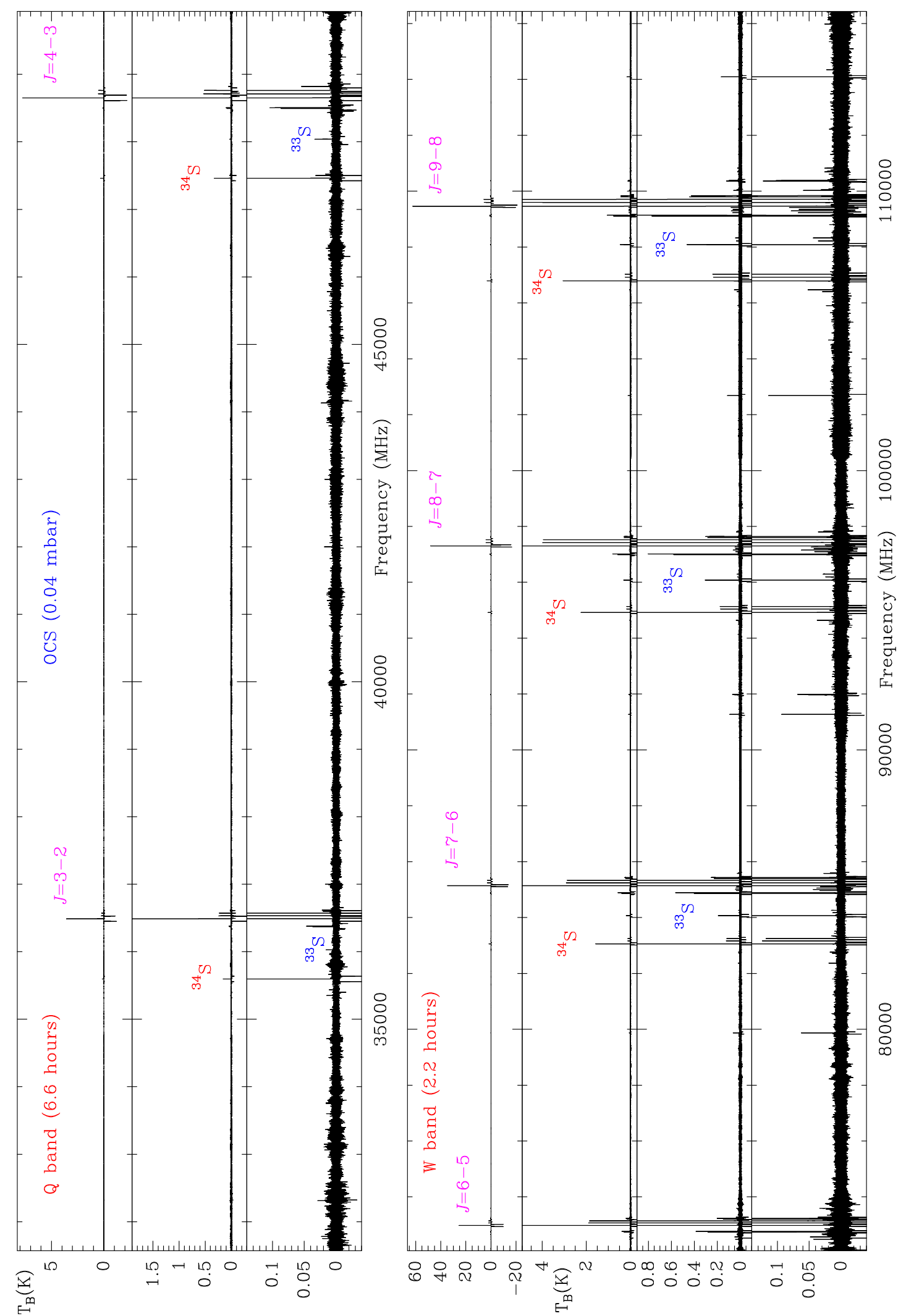

Fig. C.1. $Q$-band (top panels) and $W$-band (bottom panels) observations of OCS at a total pressure of 0.04 mbar in the chamber. The frequency switching mode has been used with a throw of $37 \mathrm{MHz}$. The different panels for each band always show the same frequency range but with a different intensity scale. The observing time per $W$ sub-band (WL, WM, and WH) is $2.2 \mathrm{~h}$. Hence, the $Q$ band has been observed for $6.6 \mathrm{~h}$. The rotational transitions of the main isotopologue are indicated. Features corresponding to $\mathrm{OC}^{34} \mathrm{~S}$ and $\mathrm{OC}^{33} \mathrm{~S}$ are also indicated. A zoom-in on the $J=9 \rightarrow 8$ transition is shown in Fig. 12. The same transition, but in a narrower frequency range, of $\mathrm{OC}^{34} \mathrm{~S}, \mathrm{OC}^{33} \mathrm{~S}, \mathrm{O}^{13} \mathrm{CS}, \mathrm{O}^{13} \mathrm{C}^{34} \mathrm{~S},{ }^{17} \mathrm{OCS}$, ${ }^{18} \mathrm{OCS}$, and $\mathrm{OC}^{36} \mathrm{~S}$ is shown in Fig. C.2. The different isotopologues of OCS are observed in natural abundance. The negative features seen in the different panels are produced by the folding of the frequency switched data and appear at $\pm 37 \mathrm{MHz}$ of each rotational transition. The data shown in this figure have been smoothed by a two-channel box procedure. 
J. Cernicharo et al.: Broad-band rotational spectroscopy for laboratory astrophysics
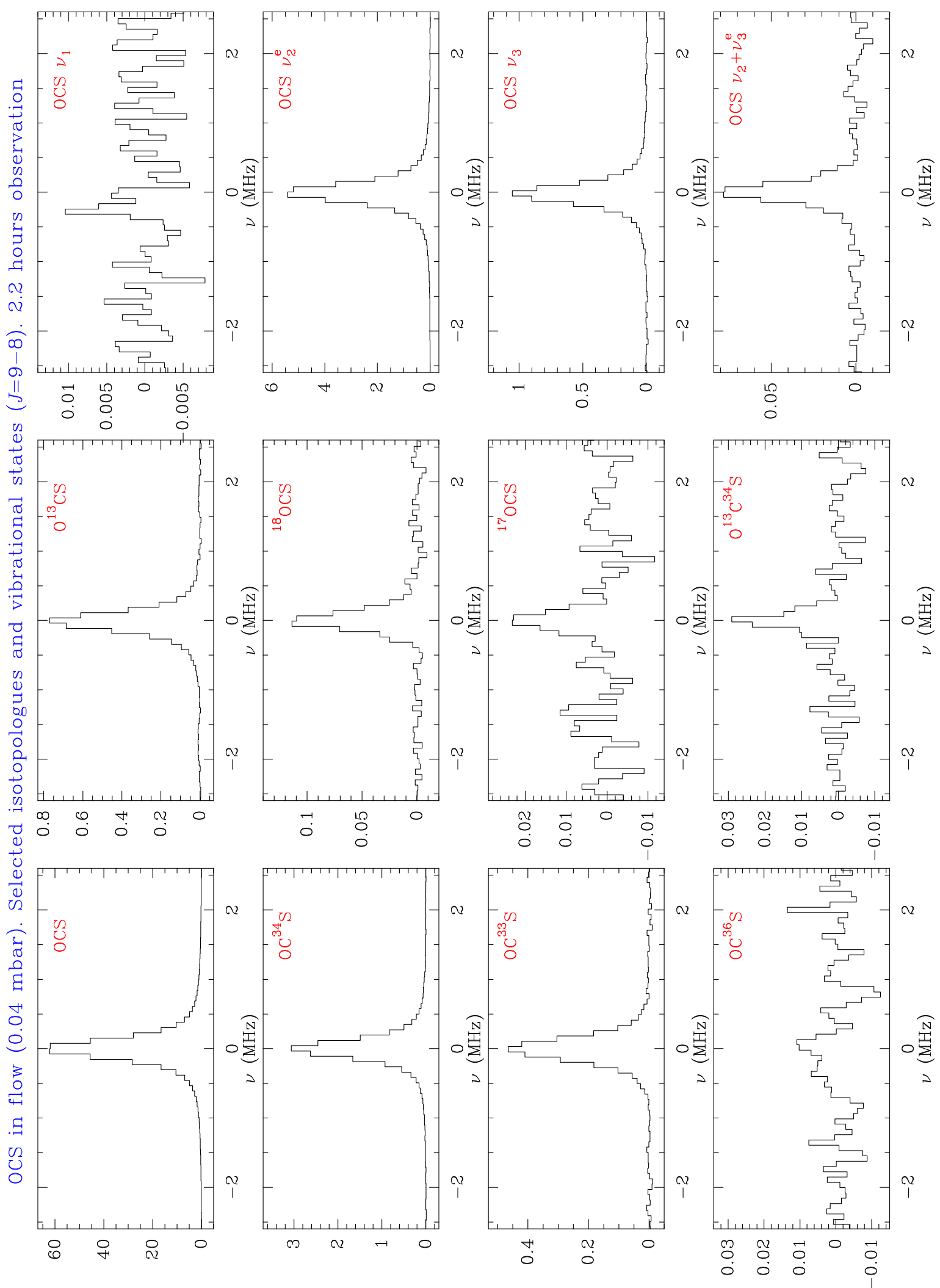

Fig. C.2. Observation of OCS at a total pressure of 0.04 mbar in the chamber using the frequency switching observing mode. Each panel shows the $J=9 \rightarrow 8$ transition of different isotopologues of OCS. All these lines are located in the WH sub-band, which has a total observing time of $2.2 \mathrm{~h}$ (see also Figs. C.1 and 12). The data shown in this figure have been smoothed by a two-channel box procedure. 


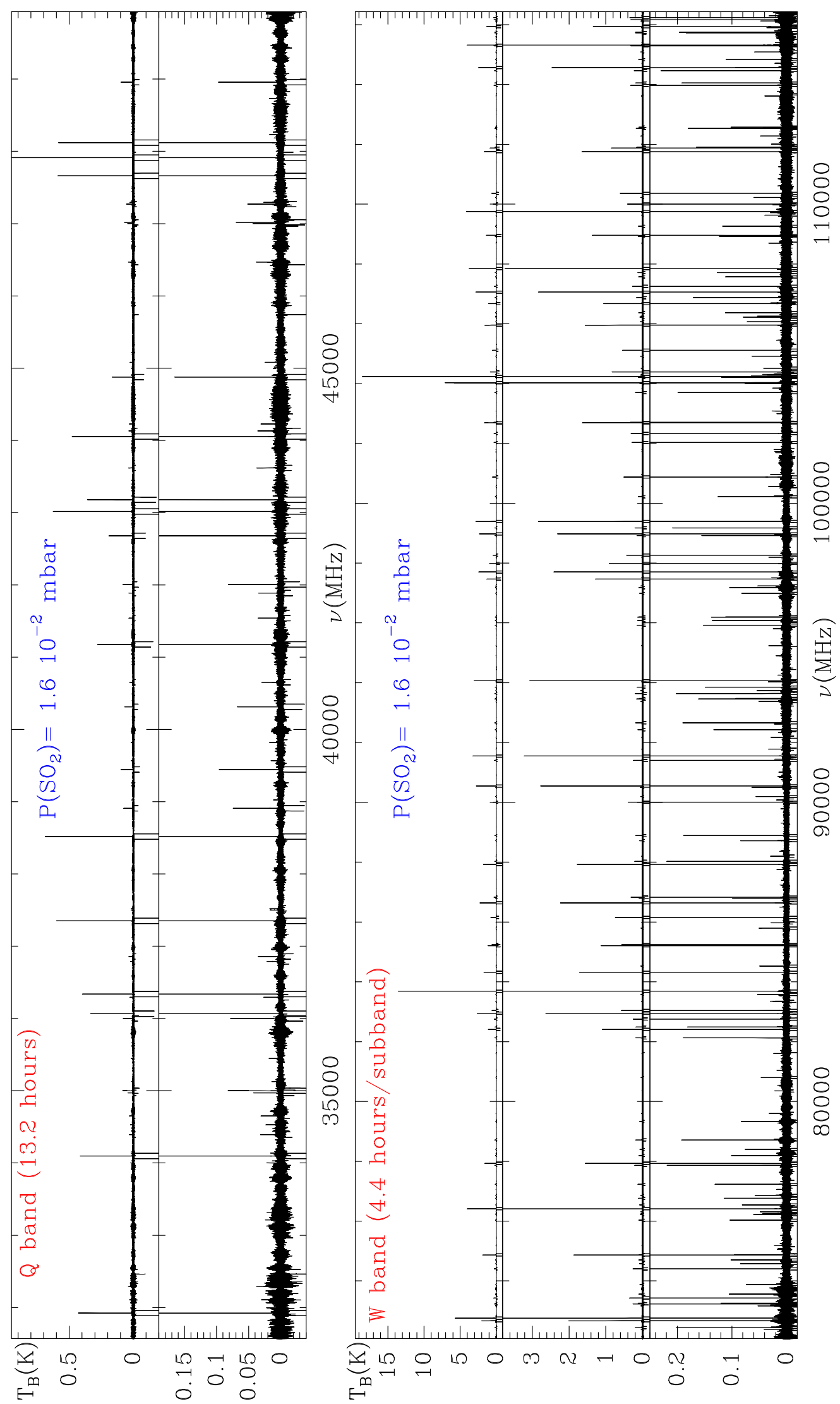

Fig. C.3. $Q$-band (top panels) and $W$-band (bottom panels) observations of $\mathrm{SO}_{2}$ at a total pressure of 0.016 mbar in the chamber. The frequency switching mode has been used with a throw of $37 \mathrm{MHz}$. The different panels for each band always show the same frequency range but with different intensity scales. The total observing time per $W$ sub-band (WL, WM, and WH) is $4.4 \mathrm{~h}$. Hence, the $Q$ band has been observed for $13.2 \mathrm{~h}$. The negative features seen in the different panels are produced by the folding of the frequency switched data and appear at $\pm 37 \mathrm{MHz}$ of each rotational transition. The data shown in this figure have been smoothed by a two-channel box procedure. 
J. Cernicharo et al.: Broad-band rotational spectroscopy for laboratory astrophysics

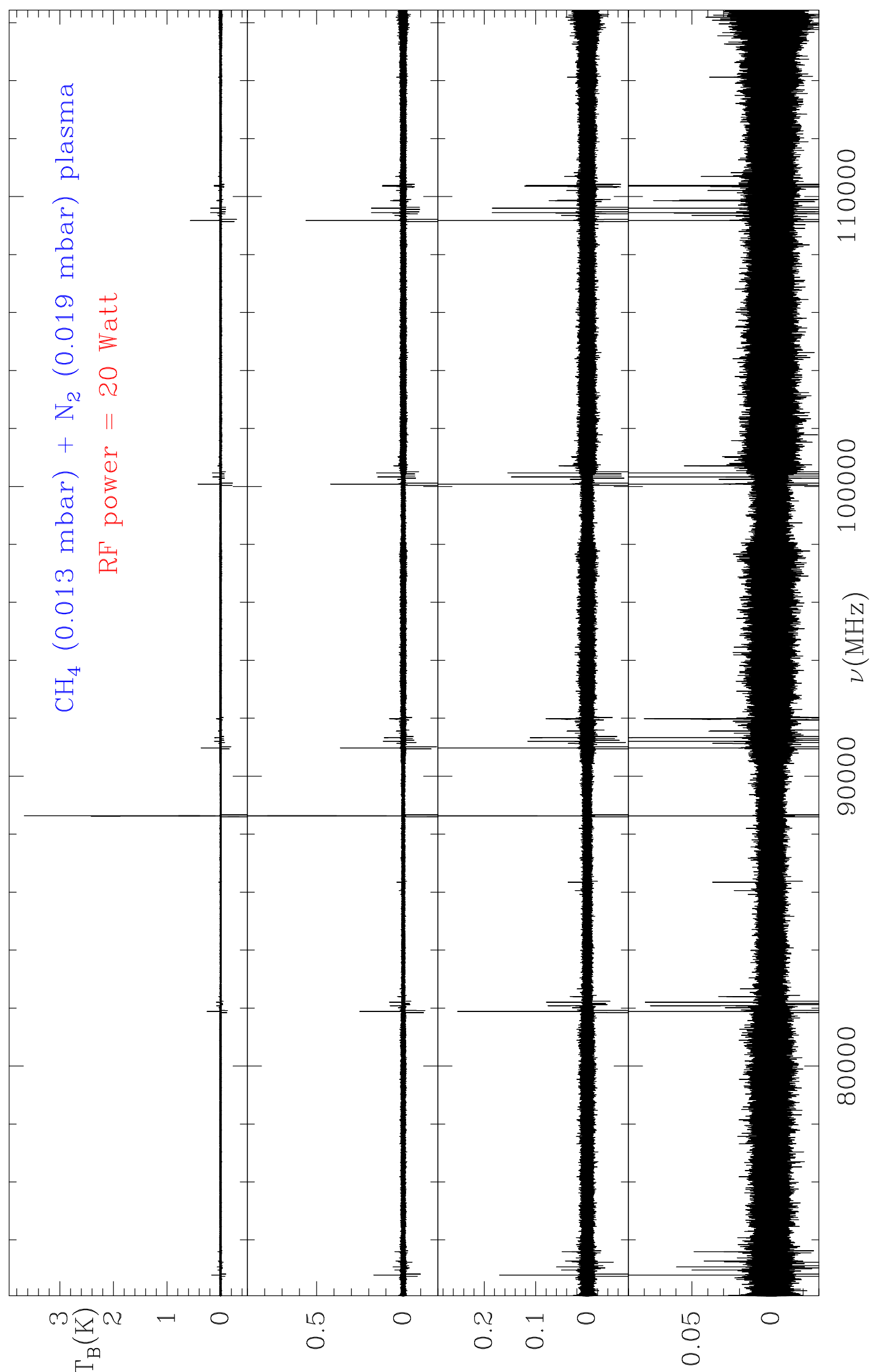

Fig. C.4. $W$-band spectrum of a $\mathrm{CH}_{4}$ and $\mathrm{N}_{2}$ plasma at a total pressure of $32.4 \times 10^{-3}$ mbar and a RF power of $20 \mathrm{~W}$. The total integration time was $40 \mathrm{~min}$ per $W$ sub-band. The observations were performed in frequency switching mode. The strongest feature corresponds to HCN. The features above the noise level correspond to $\mathrm{HC}_{3} \mathrm{~N}$ in the ground state and in the $v_{7}$ and $2 v_{7}$ modes. Many weak features correspond to $\mathrm{CH}_{3} \mathrm{CN}$. The frequency domain $82-91 \mathrm{GHz}$ is observed in both WL and WM sub-bands, and shows a better $\mathrm{S} / \mathrm{N}$. The same happens with the range 95.5-101 GHz that is observed in both WM and WH sub-bands. 


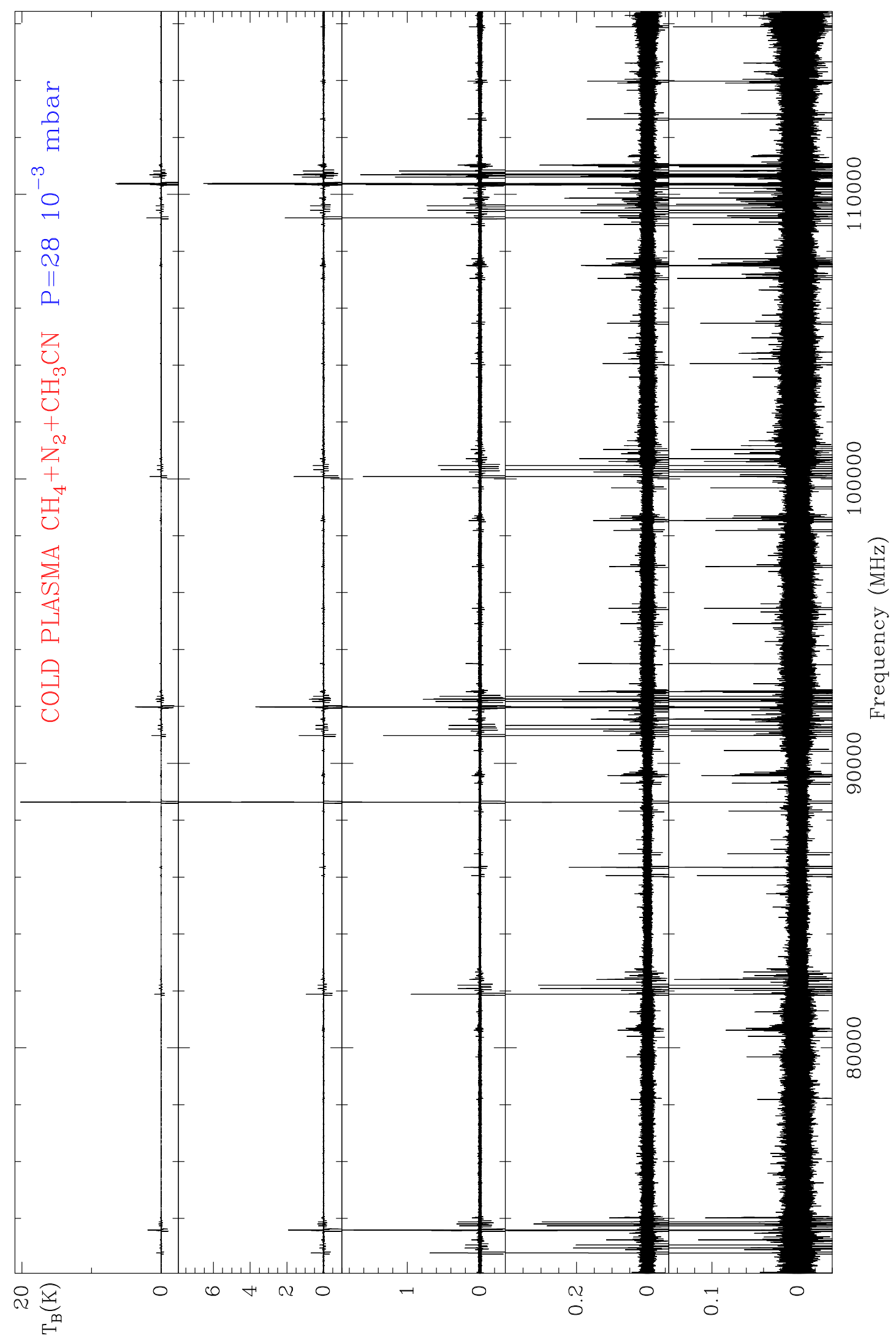

Fig. C.5. $W$-band spectrum of a $\mathrm{CH}_{4}, \mathrm{~N}_{2}$, and $\mathrm{CH}_{3} \mathrm{CN}$ plasma at a total pressure of $28 \times 10^{-3}$ mbar. The RF power is $35 \mathrm{~W}$. The observing time per $W$ sub-band is $26 \mathrm{~min}$. Only the $\mathrm{CH}_{3} \mathrm{CN}$ precursor shows rotational transitions, since $\mathrm{N}_{2}$ and $\mathrm{CH}_{4}$ have no permenent electric dipole moment. We note, however, the forest of lines appearing in this short integration time. The strong feature around $88631 \mathrm{MHz}$ corresponds to HCN. Other polar species produced in the plasma are $\mathrm{HC}_{3} \mathrm{~N}, \mathrm{CH}_{2} \mathrm{CHCN}, \mathrm{CH}_{3} \mathrm{CH}_{2} \mathrm{CN}$, and $\mathrm{CN}$ (this one is only observed in the optical spectrum). A zoom-in on the $J=1 \rightarrow 0$ line of $\mathrm{HCN}$ and its isotopologues is shown in Fig. 15. The frequency region around the $J=12 \rightarrow 11$ lines of $\mathrm{HC}_{3} \mathrm{~N}$ is shown in Fig. C.6. 
J. Cernicharo et al.: Broad-band rotational spectroscopy for laboratory astrophysics
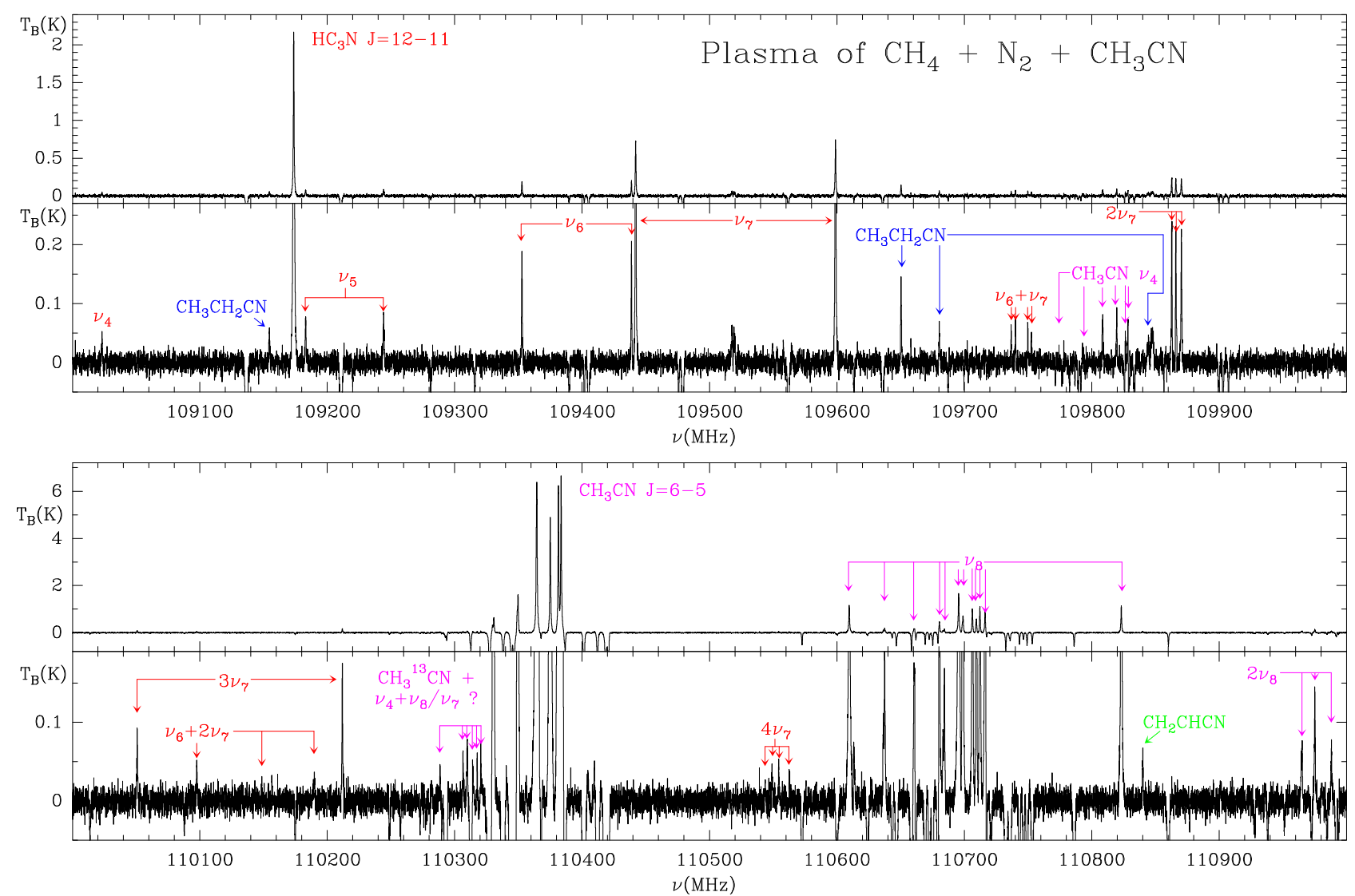

Fig. C.6. The $J=12 \rightarrow 11$ transition of $\mathrm{HC}_{3} \mathrm{~N}$ in its ground state and several vibrational modes, and the $J=6 \rightarrow 5$ of $\mathrm{CH}_{3} \mathrm{CN}_{\text {also in the ground }}$ state and the $v_{8}, 2 v_{8}$, and other possible vibrational modes. For $\mathrm{HC}_{3} \mathrm{~N}$ these vibrational states are $v_{7}, 2 v_{7}, 3 v_{7}, 4 v_{7}, v_{4}, v_{5}, v_{6}, v_{5}+v_{7}, v_{6}+v_{7}$, and $v_{6}+2 v_{7}$. The whole spectrum is shown in Fig. C.5.

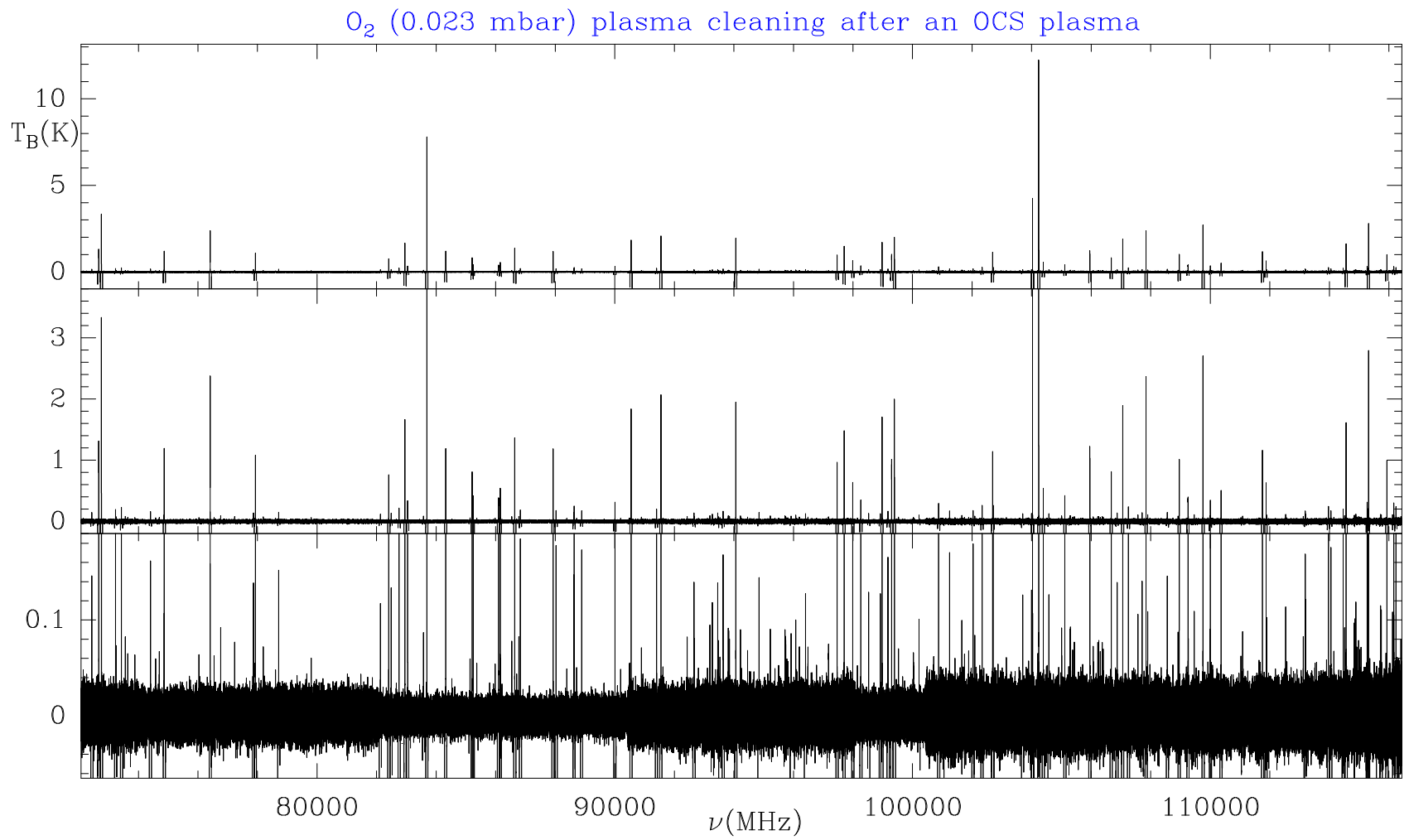

Fig. C.7. Observed spectrum in $W$ band during cleaning of the cell walls with $\mathrm{O}_{2}\left(P=0.023\right.$ mbar) plasma. Lines are due to $\mathrm{SO}_{2}, \mathrm{~S}_{2} \mathrm{O}, \mathrm{OCS}, \mathrm{CS}$, $\mathrm{CO}$, and SO (see Sect. 4.5). 